\title{
Solubility Temperature Dependence Predicted from 2D Structure
}

\author{
Alex Avdeef \\ in-ADME Research, 1732 First Avenue \#102, New York, NY 10128 USA \\ Corresponding Author E-mail: Alex@in-ADME.com; Tel.: +1-646-678-5713 \\ Received: November 20, 2015; Revised: December 23, 2015; Published: December 30, 2015
}

\begin{abstract}
The objective of the study was to find a computational procedure to normalize solubility data determined at various temperatures (e.g., $10-50^{\circ} \mathrm{C}$ ) to values at a "reference" temperature (e.g., $25^{\circ} \mathrm{C}$ ). A simple procedure was devised to predict enthalpies of solution, $\Delta H_{\text {sol }}$ from which the temperature dependence of intrinsic (uncharged form) solubility, $\log S_{0}$, could be calculated. As dependent variables, values of $\Delta H_{\text {sol }}$ at $25^{\circ} \mathrm{C}$ were subjected to multiple linear regression (MLR) analysis, using melting points $(\mathrm{mp})$ and Abraham solvation descriptors. Also, the enthalpy data were subjected to random forest regression (RFR) and recursive partition tree (RPT) analyses. A total of 626 molecules were examined, drawing on 2040 published solubility values measured at various temperatures, along with 77 direct calorimetric measurements. The three different prediction methods (RFR, RPT, MLR) all indicated that the estimated standard deviations in the enthalpy data are 11-15 kJ moll, which is concordant with the $10 \mathrm{~kJ} \mathrm{~mol}^{1}$ propagation error estimated from solubility measurements (assuming $0.05 \log \mathrm{S}$ errors), and consistent with the $7 \mathrm{~kJ} \mathrm{~mol}^{1}$ average reproducibility in enthalpy values from interlaboratory replicates. According to the MLR model, higher values of $\mathrm{mp}, \mathrm{H}$-bond acidity, polarizability/dipolarity, and dispersion forces relate to more positive (endothermic) enthalpy values. However, molecules that are large and have high $\mathrm{H}$-bond basicity are likely to possess negative (exothermic) enthalpies of solution. With $\log \mathrm{S}_{0}$ values normalized to $25^{\circ} \mathrm{C}$, it was shown that the interlaboratory average standard deviations in solubility measurement are reduced to $0.06-0.17 \log$ unit, with higher errors for the least-soluble druglike molecules. Such improvements in data mining are expected to contribute to more reliable in silico prediction models of solubility for use in drug discovery.
\end{abstract}

\section{Keywords}

Intrinsic solubility; enthalpy of solution; van't Hoff equation; solubility data mining; interlaboratory variance

\section{Introduction}

This study concerns with the prediction of the temperature dependence of intrinsic solubility $\left(S_{0}\right)$ of molecules [1,2]. A recent survey of 4557 equilibrium solubility measurements ("4557" set) of druglike (mostly ionizable) molecules in aqueous media suggested a two-step procedure to reduce the interlaboratory variance 
in solubility data mining [3]. First, data from ionizable molecules can be adjusted for $\mathrm{pH}$ effects by calculating the intrinsic solubility, $S_{0}$, based on the ionization constant, $\mathrm{p} K_{\mathrm{a}}$, and the reported water solubility, $S_{\mathrm{w}}$. Second, data can be normalized for temperature effects, by transforming solubility measurements performed at various temperatures (e.g., $10-50{ }^{\circ} \mathrm{C}$ ) to those at a benchmark value of $25^{\circ} \mathrm{C}$. With these two steps, it was thought to be possible to reduce the often cited interlaboratory reproducibility of $0.5-0.7$ log unit (or even higher) [4-7] down to near 0.15 [3]. Such improvements in data mining are expected to yield more reliable in silico prediction models of solubility for use in drug discovery.

However, the procedure of "normalizing" temperature effects was not articulated [3]. Qualitatively, it is generally expected that equilibrium solubility of most druglike molecules increases with increasing temperature (with exceptions). As far as we know, there has not been a report of a quantitative procedure where the temperature dependence of solubility is predicted from the two-dimensional (2D) structure of molecules.

Reported aqueous solubility values are mainly clustered around room and physiological temperatures: $23 \pm 3(78 \%)$ and $37 \pm 5{ }^{\circ} \mathrm{C}(22 \%)$, respectively [3]. For mostly simple molecules, there are some secondary compilations of temperature dependent solubility (e.g., Handbook of Aqueous Solubility Data by Yalkowsky et al. [8]). However, for druglike molecules, large published databases of temperature-dependent solubility are scarce. Although our focus is to improve data mining quality of solubility measurements, knowledge of the temperature dependence of solubility has a number of other practical applications.

Being able to estimate the solubility temperature dependence could allow for improved planning in early formulation studies. For example, Ismailos et al. [9] described the unusual solubility behavior of low-soluble cyclosporin $\mathrm{A}$ in aqueous media, where the enthalpy of solution, $\Delta H_{\mathrm{sol}}$, was determined to be about $-53 \mathrm{~kJ} \mathrm{~mol}^{-1}$, indicating an exothermic process: as temperature is raised, the solubility decreases. In contrast, most drug molecules are characterized by endothermic $\Delta H_{\text {sol }}$, with typical values ranging from +20 to $+50 \mathrm{~kJ} \mathrm{~mol}^{-1}$, often with uncertainty of nearly $10 \mathrm{~kJ} \mathrm{~mol}^{-1}$. The oral dosage form of cyclosporin A consisted of making a dilution with milk immediately before oral administration. However, the observed bioavailability was incomplete and erratic. To increase absorption, it was suggested [9] that a refrigerated sample of milk might be used, to keep more of the drug in solution $\left(\sim 90 \mu \mathrm{g} \mathrm{mL}^{-1}\right.$ at $\left.4{ }^{\circ} \mathrm{C}\right)$, compared to the amount dissolved in a room-temperature mixture $\left(\sim 20 \mu \mathrm{g} \mathrm{mL} L^{-1}\right.$ at $\left.25^{\circ} \mathrm{C}\right)$.

In the present study we have devised a simple procedure (similar to that used to predict the temperature dependence of $p K_{a}$ values [10]) to predict the temperature dependence of $\log S_{0}$ from the van't Hoff relationship. As dependent variables, enthalpies of solution at $25^{\circ} \mathrm{C}, \Delta H_{\text {sol }}$, were subjected to multiple linear regression (MLR) analysis, using melting point $(\mathrm{mp})$ and the Abraham $[11,12]$ six (" $5+1$ ": five traditional + product of $\mathrm{H}$-bond acidity and basicity) solvation descriptors. Also, the data were analyzed by the random forest regression (RFR) and recursive partition tree (RPT) methods. A total of 626 molecules were examined, drawing on 2040 published solubility values measured at various temperatures.

\section{Theory}

The integration of the van't Hoff differential equation over a small temperature range, between $T_{1}$ and $T_{2}$, assuming the change in standard enthalpy of solution, $\Delta H_{\text {sol }}{ }^{0}\left(\mathrm{~kJ} \mathrm{~mol}^{-1}\right)$, to be approximately independent of temperature, produces: 


$$
\log S_{2}=\log S_{1}-\frac{\Delta H_{s o l}^{0}}{2.303 R} \cdot\left(\frac{1}{T_{2}}-\frac{1}{T_{1}}\right)
$$

where $S$ is the solubility, $T$ is the absolute temperature $(\mathrm{K})$, and the gas constant is $R=8.314 \times 10^{-3} \mathrm{~kJ} \mathrm{~mol}^{-1} \mathrm{~K}^{-1}$. Equation (1) allows one to convert log $S$ determined at $T_{1}$ in a narrow temperature range (e.g., $\approx 25-37{ }^{\circ} \mathrm{C}: T_{1} \approx$ 298.15 to $310.15 \mathrm{~K}$ ) to a value at a reference temperature (e.g., $25^{\circ} \mathrm{C}: T_{2}=298.15 \mathrm{~K}$ ), provided the standard enthalpy of solution at $25^{\circ} \mathrm{C}, \Delta H_{\text {sol }}{ }^{0}$, is known.

The enthalpy value can be determined calorimetrically or from the temperature dependence of log $S$. That is, if one plots $\log S$ vs. $1 / T$ for a series of measurements at different $T$, the slope of the plotted curve evaluated at $25{ }^{\circ} \mathrm{C}$ is equal to $-\Delta H_{\text {sol }}{ }^{0} /(2.303 R)$. Often such plots show some curvature, since $\Delta H_{\text {sol }}{ }^{0}$ is slightly temperature dependent, but as a first approximation one may assume linearity if the temperature interval is small (e.g. $\approx 25-37^{\circ} \mathrm{C}$ ).

Enthalpy values determined by the slope method are subject to high uncertainty, depending on the precision of the solubility measurements and the temperature spanned. As an example, assume two solubility measurements were performed at 25 and $37{ }^{\circ} \mathrm{C}$, and that the reproducibility in each solubility measurement is 0.05 log unit. On rearranging Equation (1) and casting it in the propagation-of-error form (SD = standard deviation),

$$
S D\left(\Delta H_{\text {sol }}^{0}\right)=\frac{2.303 R}{\left(\frac{1}{T_{2}}-\frac{1}{T_{1}}\right)} S D\left(\log S_{2}-\log S_{1}\right)=147 \frac{\mathrm{kJ}}{\mathrm{mol}}\left[(0.05)^{2}+(0.05)^{2}\right]^{1 / 2}=10 \frac{\mathrm{kJ}}{\mathrm{mol}}
$$

One of the objectives of this study is to transform intrinsic solubility, $\log S_{0}{ }^{\top}$, measured at $T$, to $\log S_{0}{ }^{\text {ref }}$, the solubility at "reference" temperature $298.15 \mathrm{~K}\left(25^{\circ} \mathrm{C}\right)$. Rearrangement of Equation (1) produces:

$$
\log S_{0}^{r e f}=\log S_{0}^{T}-0.1752 \Delta H_{s o l}^{0} \cdot\left(1-\frac{298.15}{T}\right)
$$

For example, if $\Delta H_{\text {sol }}{ }^{0}=+30 \mathrm{~kJ} / \mathrm{mol}, \mathrm{T}=310.15 \mathrm{~K}\left(37^{\circ} \mathrm{C}\right)$, and the reference temperature is selected as $25^{\circ} \mathrm{C}$, then $\log S_{0}{ }^{\text {ref }}=\log S_{0}{ }^{310.15}-0.203$. That is, the molecule is 1.6 times less soluble at $25^{\circ} \mathrm{C}$, compared to that at $37^{\circ} \mathrm{C}$.

Thermodynamic standard state definition traditionally is based on concentrations being extrapolated to zero (unit activity) value. However, most of the measured solubility values used in Equation (1) was determined in saturated water solutions, with many molecules undergoing some degree of ionization. It is assumed that by working with intrinsic solubility values (referring to solubility of molecules in the uncharged form), some of the consequences of non-unit activity considerations in the definition of the standard state $\Delta H_{\text {sol }}{ }^{0}$ may be mitigated, especially for sparingly soluble molecules. To highlight that intrinsic solubility is not determined at the standard (zero-concentration, unit activity) state, $\Delta H_{\text {sol }}$ (without the superscript) will be associated with solubility measurement in saturated solutions.

\section{Methods}

A total of 626 enthalpies of solution were gathered, either directly from calorimetric data (12\%) or indirectly from temperature-dependent solubility data (88\%). 
Van't Hoff Analysis of Temperature-Dependent Solubility Data

When available, the enthalpies of solution were taken directly from primary publications. Otherwise, the temperature-dependent intrinsic solubility values for each molecule were fitted in this study to the linear equation:

$$
\log S_{0}=\mathrm{a}+\mathrm{b} / T
$$

The value of $\Delta H_{\text {sol }}$ was set equal the slope, b, multiplied by $-2.303 R$ (cf., Equation 1 ). The solubility values in the units of the source publications (molality or molarity) were used in Equation (4). (Mole fraction units were converted to molality prior to fitting.)

\section{Calorimetric Data Sources}

Calorimetrically measured enthalpies of solution are considered to be more accurate than those derived from solubility as a function of temperature. A search located 77 molecules with reported calorimetric enthalpies of solution, all from primary literature. Table A1 in the Appendix indicates which enthalpies were the calorimetrically determined.

\section{Solubility Data Sources}

Yalkowsky et al. [8] Handbook of Aqueous Solubility Data is a reliable and convenient secondary source of temperature-dependent $S_{w}$ values of relatively simple molecules. The data were measured by the saturation shake-flask method [3], and are expected to have good precision. A search led to 653 suitable $S_{w}$ values for 195 molecules, with 2-6 solubility-temperature points per molecule, in the interval $20 \pm 5$ to $41 \pm 7{ }^{\circ} \mathrm{C}$. The handbook values mainly ranged from about -0.1 to -3.5 log molarity units (mean -1.8 ).

An additional 1387 measurements as a function of temperature were gathered from primary publications for 354 molecules, with measurements based on the shake-flask (traditional and various miniaturized variants) and two potentiometric (DTT and CheqSol) methods, as described elsewhere [3]. This set included more druglike molecules than that from the handbook, and ranged in log $S_{0}$ from +2.7 to -8.4 (mean -3.0). Table A1 indicates which enthalpies were calculated from solubility data.

\section{Conversion of Water Solubility $\left(S_{w}\right)$ to Intrinsic Solubility $\left(S_{0}\right)$}

The pDISOL-X program (www.in-adme.com/pdisol x.html, in-ADME Research) was used to convert nominally unbuffered water solubility $\left(S_{\mathrm{w}}\right)$ values with unspecified $\mathrm{pH}$ to intrinsic values $\left(S_{0}\right)$ and to calculate the saturation $\mathrm{pH}\left(\mathrm{pH}_{\text {sat }}\right.$ ), as described by Völgyi et al. [13] and others [14-16]. Since no instances of ambient $\mathrm{CO}_{2}$ concentration were reported in solubility measurement studies, for practically-insoluble bases with $\mathrm{p} K_{\mathrm{a}}>8$ (e.g., terfenadine, clofazimine), $\left[\mathrm{CO}_{2}\right]=10 \mu \mathrm{M}$ was assumed in the calculation of $S_{0}$. Sparingly-soluble acids were less affected by ambient levels of $\mathrm{CO}_{2}$. Also, in the calculations, it was necessary to assume that the Henderson-Hasselbalch relationship was valid [3], except when multiple-pH buffer $S_{\mathrm{pH}}$ values (log $S$ vs. $\mathrm{pH}$ ) data were available to derive the corresponding $S_{0}$ values.

\section{Data Types and Exclusions}

It was suspected that enthalpy of solution might depend on the acid-base properties of the molecule, as 
was found in the study of the temperature dependence of $\mathrm{p} K_{\mathrm{a}}$ values [10]. The enthalpy of solution might be influenced by differences between the solvation effects in the proton releasing and gaining processes, or by the electron density of the different functional groups. Consequently, the selected compounds were assigned indicator indices: $I_{A}, I_{B}, I_{A B}$, and $I_{N}$, with a unit value indicating that a molecule is an acid, base, ampholyte, or neutral, respectively, and zero otherwise.

Given the multitude of methods and conditions used to measure solubility [3], from which most of the enthalpy of solution values were derived here, and, as mentioned above, such enthalpy values are expected to be sensitive to errors in the log $S$ vs. $1 / T$ data, intralaboratory enthalpy variances are expected to be smaller than interlaboratory variances. About $6 \%$ of the $\log S$ measurements were done at two temperatures, each result reported from a different laboratory (" $n=2$, different labs" set), potentially representing the leastreliable slope-calculated enthalpy values. By contrast, in $73 \%$ of the studies, all of the temperature-dependent log S measurements for a given molecule come from the same laboratory (e.g., the curated "653-set" from the Yalkowsky et al. handbook [8]). The latter "one source" temperature-solubility data are expected to lead to the most-reliable calculated enthalpy values.

For each molecule, the $\log S$ values were separated into two groups: those determined at $t<30{ }^{\circ} \mathrm{C}$ ("room temperature set", RT) and those at $t \geq 30{ }^{\circ} \mathrm{C}$ ("physiological temperature set", PT). For each grouping, the standard deviation $\left(\mathrm{SD}_{\mathrm{RT}}, \mathrm{SD}_{\mathrm{PT}}\right)$ values were calculated for each molecule based on replicate measurements. The average of all SD values was 0.18 log unit for the dataset. A given molecule with SD greatly exceeding the average value would be excluded from the training and test sets.

Since we are only using 2D molecular descriptors, different solvates of a given compound were lumped together with nonsolvates for the compound (when this information was available).

Of the 626 enthalpies of solution located/calculated, some values were suspected to be unreliable. We applied three filters to systematically exclude those points from training and test sets from the start, but their predicted $\Delta \mathrm{H}_{\text {sol }}$ values were calculated nevertheless (Table A1).

Three systematic exclusion criteria were:

- The " $n=2$, different labs" data were excluded from model training and testing.

- If for a given molecule, $\mathrm{SD}_{\mathrm{RT}}$ or $\mathrm{SD}_{\mathrm{PT}}>0.3$, the data were excluded from the model training and testing.

- Solubility-derived enthalpies were excluded if slope-calculated values of $\Delta H_{\text {sol }}<-80$ or $>+80 \mathrm{~kJ} \mathrm{~mol}^{-1}$, based on the observation that in the best-quality (calorimetric) data, enthalpy data ranged from -37 to $+50 \mathrm{~kJ} / \mathrm{mol}$. We put an arbitrary cut-off of $\pm 80 \mathrm{~kJ} \mathrm{~mol}^{-1}$ in the expectation that erratic values calculated from solubility-temperature data would less likely enter the training set.

On applying the above criteria, 55 compounds were excluded. Thus 571 molecules were selected for the training and test sets. In the 571-set, $43 \%$ were acids, $13 \%$ were bases, $27 \%$ were ampholytes, and $17 \%$ were nonionizable molecules. The above ionization type distribution is not ideally suggestive of druglike molecules, which include more bases than acids. For the 77 calorimetrically measured $\Delta H_{\text {sol, }}$ the average value is $+13 \mathrm{~kJ} \mathrm{~mol}^{-1}$, with values ranging from -37 to $+50 \mathrm{~kJ} \mathrm{~mol}^{-1}$. By comparison, the log S vs. $\mathrm{T}$ derived $\Delta \mathrm{H}_{\text {sol }}$ have the average value $+21 \mathrm{~kJ} / \mathrm{mol}$, and the much wider range from -97 to $+120 \mathrm{~kJ} / \mathrm{mol}$ (before exclusions).

Variances of Replicate $\Delta H_{\text {sol }}$

In the 626 enthalpy set, there were 99 instances of replicate $\Delta H_{\text {sol }}$ values, measured in different 
laboratories. The average standard deviation in the replicates was $7 \mathrm{~kJ} \mathrm{~mol}^{-1}$, in line with expectations that the quality of the data were limited by the expected uncertainties in log $S$ measurement (error $\geq 0.05 \log$ unit), as considered in the Theory section.

Prediction of Enthalpy of Solution

The random forest regression (RFR) method is a powerful and easy-to-use new statistical (albeit somewhat of a "black box") tool which can cope with very large numbers of correlated descriptors, something that is not possible with traditional multiple linear regression (MLR) methods. It was thus a good starting point for our enthalpy data analysis. Using RFR could reveal sensitive descriptors that might not have been otherwise obvious. The recursive partition tree analysis provides a simple alternative view of the characteristics of the data in relation to the descriptors. However, the important advantage of the MLR analysis is that the results are thought to be easier to understand, in terms of the role of specific descriptors in the prediction of physicochemical properties, such as the temperature dependence of solubility.

The RFR modeling (Walters [17] - very useful tutorial) was first explored, starting with the 193 descriptors ("RDK" set, including lipophilicity as indicated by $\log P$ and $\log D$, connectivity and molecular shape indices, topological and electrotopological state indices, surface area contributions, partial atomic charges, $\mathrm{H}$-bond donor/acceptor counts, molecular refractivity, and other more specialized descriptors) calculated by the opensource chemoinformatics and machine-learning RDKit library of programs (Landrum et al. [18]; http://rdkit.readthedocs.org/en/latest/), combined with the six Abraham solvation descriptors [11,12], along with the acid-base indicators $\left(I_{A}, I_{B}, I_{A B}\right.$, and $\left.I_{N}\right)$, and the published melting points $(\mathrm{mp})$. Where the latter values were not found, the Lang and Bradley [19] predicted melting points in the QsarDB open repository of data and prediction tools (http://qsardb.org/repository/handle/10967/104) [20] were used. Table A1 indicates which $\mathrm{mp}$ was calculated and which was experimental.

The six ("5+1") Abraham "ABSOLV" descriptors $\left(\Sigma \alpha_{2}{ }^{\mathrm{H}}, \Sigma \beta_{2}{ }^{\mathrm{H}}, \Sigma \alpha_{2}{ }^{\mathrm{H}} \cdot \Sigma \beta_{2}{ }^{\mathrm{H}}\right.$ : H-bond acidity and basicity, and the product of the two; $\pi_{2}$ : dipolarity/polarizability; $\mathrm{R}_{2}$ : dispersion force; and $\mathrm{V}_{\mathrm{x}}$ : molar volume - see Glossary of Terms for further elaboration) were estimated from the 2D structure of molecules using ADME Boxes v4.9 program from ACD/Labs (Advance Chemistry Development, Inc., www.ACDLabs.com). Perhaps better fits might be expected from descriptors derived directly from measurements rather than from in silico predictions. However, experimentally-based ABSOLV values may not be available for all the compounds considered here.

After the RFR modeling, recursive partition tree analysis and multiple linear regression (MLR) calculations were explored, using the Algorithm Builder v.1.8 program from ACD/Labs.

SMILES representations of the 2D structures of molecules were available at the Royal Society of Chemistry ChemSpider website: http://www.chemspider.com/. ACD/ChemSketch from ACD/Labs was used to construct "SDF/mol" format 2D representations of the molecules.

\section{Model Validations}

The RFR method randomly selected $30 \%$ of the data to be test sets. The RTP method was not validated with a test set, since it was used in a qualitative way. In the MLR method, the "leave-many-out" (LMO) crossvalidation procedure ( $20 \%$ of the measurements randomly excluded in 100 different repeated combinations), where a cross-validated $\mathrm{q}^{2}$ was used to assess model predictivity. 
Table 1 - Random Forest Regression (RFR) Results for the Prediction of Enthalpies of Solution

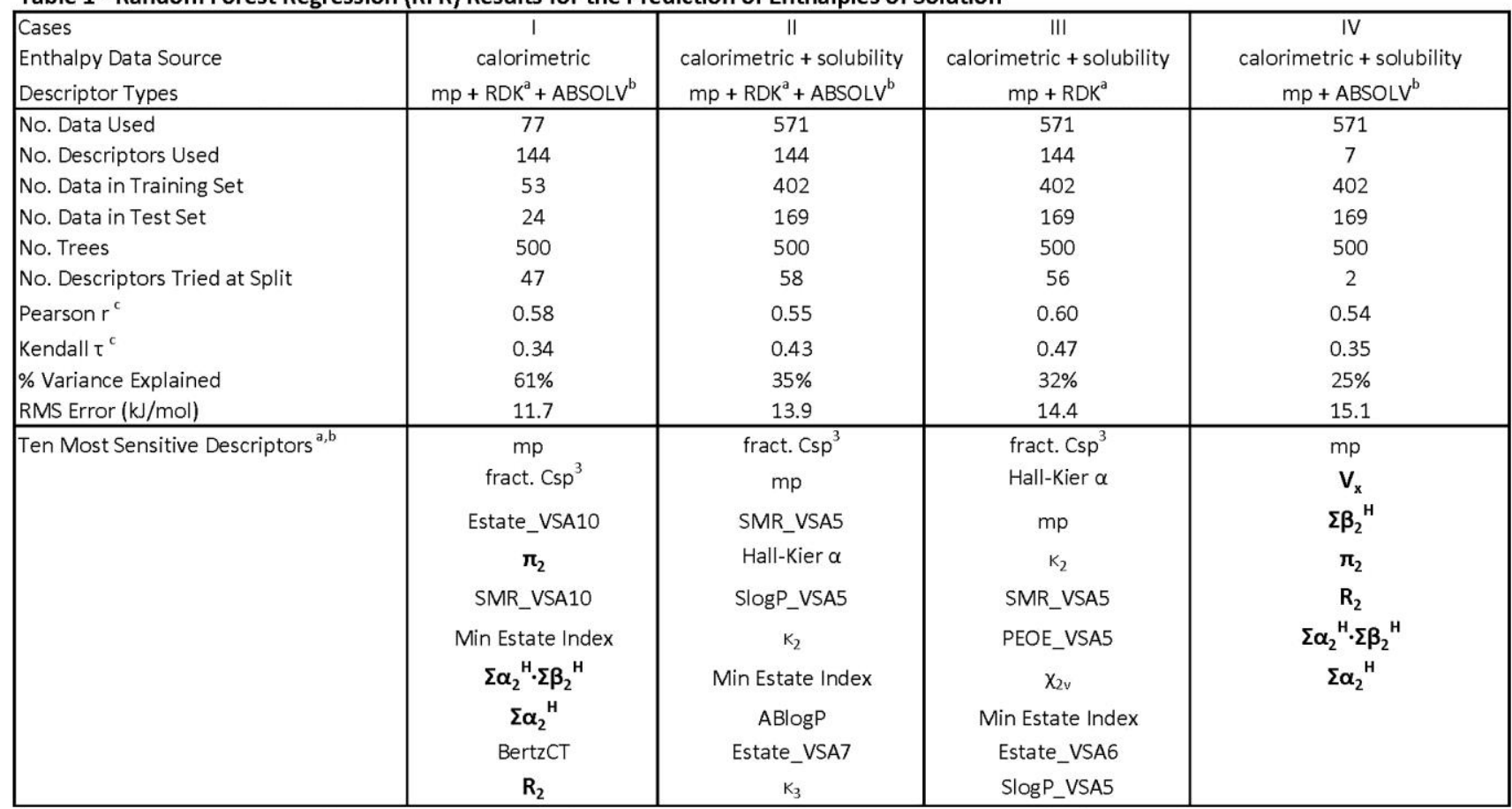

${ }^{a}$ RDKit descriptors (cf., http://www.chemcomp.com/journal/vsadesc.htm; http://www.vcclab.org/lab/indexhlp/estate.html) include:

$\alpha, \chi_{2 v}, K_{2}, K_{3} \quad$ Hall-Kier [23] connectivity and kappa molecular shape indices

ABlogP calculated octanol-water partititon coefficient, using ADME Boxes v.4.9 (www.ACDLabs.com)

BertzCT topological descriptor [24]

Estate_VSAk; Min Estate Index electrotopological state indices and surface area contributions, $k^{\text {th }}$ order [23]

fract. Csp ${ }^{3}$

PEOE_VSAK

fraction of atoms that are $\mathrm{sp}^{3}$-type carbon

SlogP_VSAK

partial charge and surface area contributions, $\mathrm{k}^{\text {th }}$ order

SMR_VSAK

octanol-water partition coefficient and surface area contributions, $k^{\text {th }}$ order [22]

molecular refractivity and surface area contributions, $\mathrm{k}^{\text {th }}$ order [22]

${ }^{b}$ ABSOLV are Abraham solvation descriptors (listed in bold) [11,12] - see Glossary of terms

'Pearson's $r$ is the product-moment correlation [17]. It can be sensitive to the outlier and distribution of data effects. The Kendall $\tau$

is a non-parametric of correlation,employing rank order of values, rather than the values directly. It is less sensitive to outliers

\section{Results and Discussion}

\section{Random Forest Regression (RFR) Analysis}

As summarized in Table 1, four different combinations were tried using random forest regression analysis: (I) calorimetric data only - $\mathrm{mp}, \mathrm{RDK}, \mathrm{ABSOLV}$ descriptors; (II) calorimetric + solubility-derived data - mp, RDK, ABSOLV descriptors; (III) like Case II, but without ABSOLV descriptors; and (IV) like Case II, but excluding RDK descriptors.

The small calorimetric set (Case I) was best predicted. The root-mean-square (RMS) error was $11.7 \mathrm{~kJ} \mathrm{~mol}^{-1}$; $61 \%$ of the variances were predicted. The 10-most sensitive descriptors are shown in Table 1 for this case. At the top of the list is $\mathrm{mp}$. Four of the six ABSOLV descriptors were among those in the top list.

The combined calorimetric + solubility-derived set (II) included all the "571-set" data and the full set of descriptors. The RMS error was slightly higher at $13.9 \mathrm{~kJ} \mathrm{~mol}^{-1} ; 35 \%$ of the variances were predicted, which suggests that the solubility-based data dragged down the overall precision of the combined data set. The $\mathrm{mp}$ 
descriptor was in the second position from the top, but there were no ABSOLV descriptors showing up in the top-10 list. The most sensitive RDK descriptors included fractions of $\mathrm{sp}^{3}$ carbon, molecular refractivity - surface area [22], log $P$ - surface area [22], various Hall-Kier electrotopological, connectivity and molecular shape indices, and $\log P[23]$.

Cases III and IV excluded either RDK or ABSOL descriptors. The statistics were similar to those of the fullcomplement of descriptors (II), with RMS errors rising slightly to 14.4 and $15.1 \mathrm{~kJ} \mathrm{~mol}^{-1}$ for the RDK (III) and the ABSOLV (IV) cases, respectively, and with accounted variances decreasing slightly. In Case IV (ABSOLV), the order of sensitivities comprised: $m p>V_{x}>\Sigma \beta_{2}{ }^{H}>\pi_{2}>R_{2}>\Sigma \alpha_{2}{ }^{H} \cdot \Sigma \beta_{2}{ }^{H}>\Sigma \alpha_{2}{ }^{H}$.

\section{Recursive Partition Tree (RPT) Analysis}

The "571-set" exclusion-filtered data (calorimetry + solubility-derived), with $\mathrm{mp}+$ ABSOLV + acid-base indicator indices $\left(I_{A}, I_{B}, I_{A B}, I_{N}\right)$ as descriptors, was next subjected to recursive partition tree analysis (Algorithm Builder v.1.8). Figure 1 shows the decision tree.

The first node automatically separated the acids from the non-acids. Within the acid set, $\mathrm{mp} \geq 303{ }^{\circ} \mathrm{C}$ further split the group into two. There are 9 molecules in the higher $\mathrm{mp}$ set, with average $\Delta H_{\text {sol }}=47 \pm 13$ $\mathrm{kJ} \mathrm{mol}^{-1}$. The compounds with the lower mp included 235 molecules, with average $\Delta H_{\text {sol }}=27 \pm 12 \mathrm{~kJ} \mathrm{~mol}^{-1}$.

For the non-acids, $\Sigma \beta_{2}{ }^{H} \geq 3.8$ split the set into two groups. The group with strong $\mathrm{H}$-bond acceptors included 8 molecules, with average $\Delta H_{\text {sol }}=-24 \pm 11 \mathrm{~kJ} \mathrm{~mol}^{-1}$, associated with an exothermic process. The 319 molecules in the weaker $\mathrm{H}$-bond basicity group were split into two subsets according to molar refractivity, $R_{2} \geq 0.47$. The 279 molecules with stronger dispersion force interaction (arising from pi- and $\mathrm{n}$-electrons of the solute) had the average $\Delta H_{\text {sol }}=19 \pm 17 \mathrm{~kJ} \mathrm{~mol}^{-1}$, whereas the 40 "hard" molecules had the lower average $2 \pm 15 \mathrm{~kJ} \mathrm{~mol}^{-1}$.

\section{Multiple Linear Regression (MLR) Analysis}

Table 2 summarizes the multiple linear regression analysis of the enthalpy data, using the mp + ABSOLV + acid-base indicator indices $\left(I_{A}, I_{B}, I_{A B}, I_{N}\right)$ descriptors.

The calorimetric case (A) again was best-fit, with $r^{2}=0.67$ and $s=10.8 \mathrm{~kJ} \mathrm{~mol}^{-1}$. The statistics were comparable to those found in the RFR analysis for the same set. Unlike the RFR method, it may be easier to interpret the contributions of each of the ABSOLV descriptors. The four acid-base indicator indices in effect acted as four different intercepts: acids and ampholytes had positive additive contributions, whereas bases and neutrals had negative contributions, in addition to those trends predicted by the mp + ABSOLV descriptors. The $\mathrm{H}$-bond basicity $\left(\Sigma \beta_{2}{ }^{\mathrm{H}}\right)$ led to negative (exothermic) enthalpy contributions, whereas $\pi_{2}$ (solute polarity/polarizability due to solute-solvent interactions between bond dipoles and induced dipoles) contributed to positive (endothermic) enthalpy values. The other ABSOLV descriptors made smaller contributions in the Case A model. Enthalpy of solution was predicted to increase with increasing melting points. Figure $2 \mathrm{a}$ shows the correlation plot for the calorimetric set. As can be seen, acids tend to have higher positive enthalpies compared to the other molecules. 


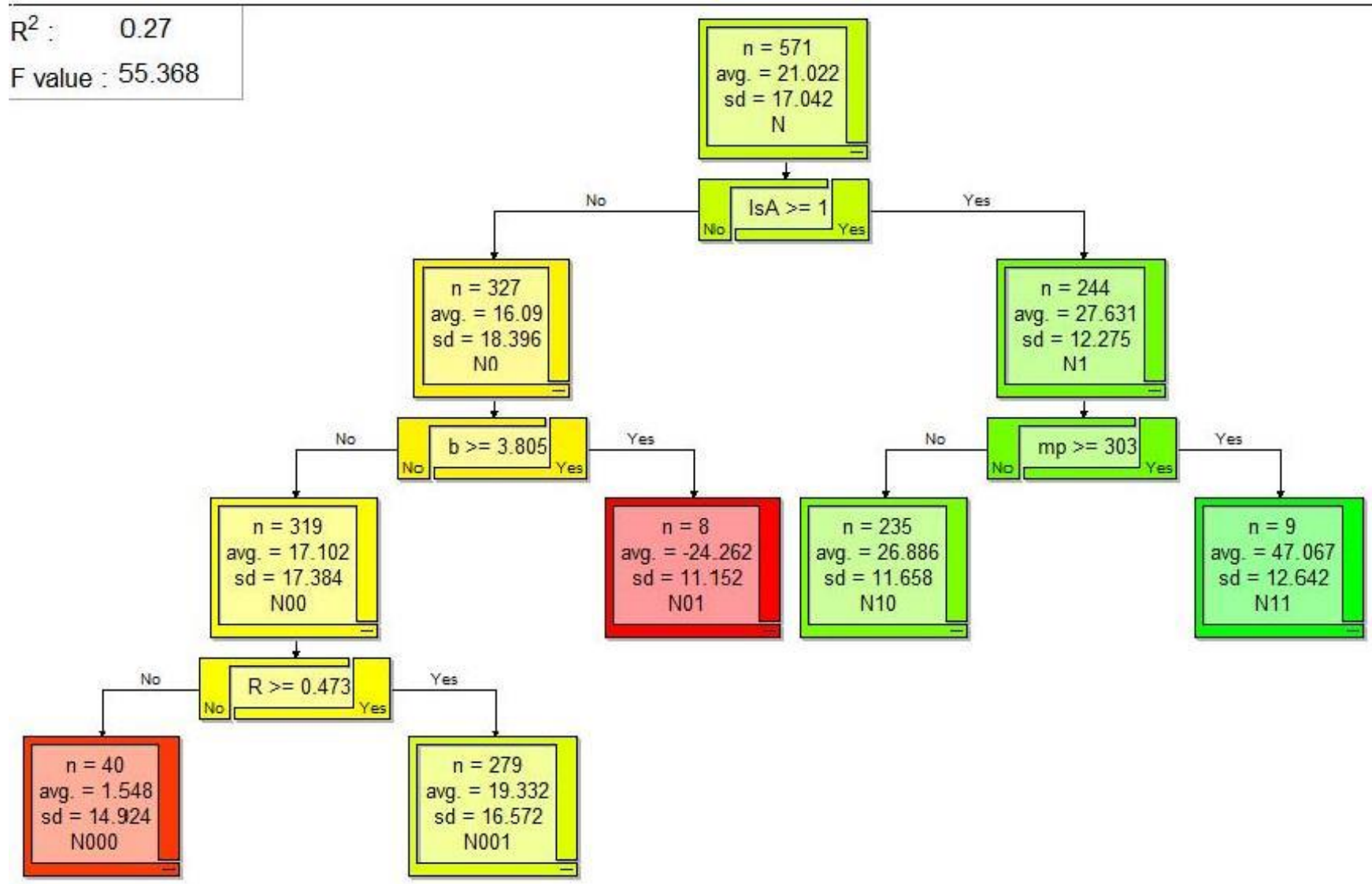

Figure 1. Recursive partition tree analysis (Algorithm Builder, v.1.8), separating acids from non-acids. For acids, $\mathrm{mp}$ is a key discriminator. For non-acids, $\mathrm{H}$-bond basicity and molar refractivity are key discriminators.

Table 2 - Multiple Linear Regression (MLR) for the Prediction of Enthalpies of Solution

\begin{tabular}{|c|c|c|c|c|c|c|c|c|c|c|c|}
\hline \multirow[b]{2}{*}{ Case Model } & \multicolumn{11}{|c|}{ MLR Descriptor Coefficients ${ }^{a}$} \\
\hline & $I_{A}$ & $I_{B}$ & $\mathrm{I}_{\mathrm{AB}}$ & $I_{N}$ & $\mathrm{mp} / 100$ & $\sum \alpha_{2}{ }^{H}$ & $\Sigma \beta_{2}{ }^{\mathrm{H}}$ & $\pi_{2}$ & $\mathrm{R}_{2}$ & $V_{x}$ & $\Sigma \alpha_{2}{ }^{\mathrm{H}} \cdot \Sigma \beta_{2}{ }^{\mathrm{H}}$ \\
\hline A (calorimetric data only) & 10.3 & -16.0 & 2.0 & -12.4 & 2.4 & 3.1 & -8.3 & 11.9 & 2.2 & -2.1 & 1.0 \\
\hline B (solubility-derived data only) & 25.9 & 19.9 & 16.6 & 18.1 & 1.5 & 2.6 & -7.4 & 4.3 & 8.3 & -7.8 & -1.3 \\
\hline$C$ (non-acids) & -- & 11.2 & 8.9 & 7.3 & 3.9 & 1.9 & -9.1 & 6.5 & 10.2 & -8.7 & -0.9 \\
\hline$D$ (acids) & 17.1 & -- & -- & -- & 2.4 & 5.8 & -1.9 & 3.0 & 2.7 & -0.4 & -4.3 \\
\hline E (acids \& non-acids) & 20.2 & 11.9 & 10.8 & 8.3 & 2.7 & 4.0 & -8.2 & 5.2 & 8.7 & -6.5 & -1.8 \\
\hline \multirow[t]{2}{*}{ Std. Error } & & \pm 3.0 & \pm 2.7 & \pm 2.7 & \pm 1.7 & & & & \pm 1.6 & \pm 1.5 & \\
\hline & \multicolumn{4}{|c|}{ MLR Statistics } & & & & & & & \\
\hline Case Model & $r^{2}$ & $\mathrm{~s}(\mathrm{~kJ} / \mathrm{mol})$ & $\mathrm{F}$ & $n$ & & & & & & & \\
\hline A (calorimetric data only) & 0.67 & 11 & 22 & 77 & & & & & & & \\
\hline$B$ (solubility-derived data only) & 0.29 & 14 & 130 & 494 & & & & & & & \\
\hline C (non-acids) & 0.30 & 16 & 48 & 327 & & & & & & & \\
\hline $\mathrm{D}$ (acids) & 0.07 & 12 & 163 & 244 & & & & & & & \\
\hline E (acids \& non-acids) & 0.31 & 14 & 135 & 571 & & & & & & & \\
\hline
\end{tabular}

${ }^{a}$ See Glossary of Terms for definition of the Abraham and acid-base indicator descriptors.

The non-calorimetric case (B) was predicted less well, compared to the above calorimetric case, just as in the case in RFR analysis. There was less acid-base differentiation, as indicated by the similarity of four I-indices. The other main difference between Cases $A$ and $B$ is that the dispersion-force $R_{2}$ played a more prominent role in Case $B$ than the dipolarity/polarizability $\pi_{2}$, with high values predicting more positive (endothermic) enthalpies. Also, compounds with larger McGowan molar volumes $\left(\mathrm{V}_{\mathrm{x}}\right)$ were associated with more negative (exothermic) enthalpies. 
The recursive partitioning tree analysis suggested that acids could be grouped separately from the non-acids. Cases $C$ and $D$ tested that idea. The acids had a muted contribution from ABSOLV descriptors (except for the $\mathrm{H}$-bond acidity), compared to the non-acids, as is indicated in Table 2. For example, a model using just the $I_{A}, m p, \Sigma \alpha_{2}{ }^{H}$, and $\Sigma \alpha_{2}{ }^{H} \cdot \Sigma \beta_{2}{ }^{H}$ descriptors yielded $r^{2}=0.04, s=$ 12.1, and $F=321$; a model using just $I_{A}$ and $\mathrm{mp}$ yielded $r^{2}=0.03, s=12.1$, and $F=$ 641. Figure 2(b) shows the correlation plot, combining the Case $C$ and $D$ sets. The acids are on the high positive enthalpy side of the plot. Most of the molecules that have very negative enthalpy values are large complex species (e.g., cyclosporine A, clarithromycin, erythromycin, ivermectin, digoxin, and digitoxin).

The calculated enthalpy of solution values in Table $A 1$ are based on Case $D$ applied to acids and Case $\mathrm{C}$ applied to non-acids. Very similar results were obtained from the application of combined Case E model (not shown). This Cases C+D method of calculation has been incorporated into the current release of $p D I S O L-X$, where intrinsic solubility, $\log S_{0}$, are now predicted both at 25 and $37^{\circ} \mathrm{C}$.

\section{Interlaboratory Errors Temperature Effect}

Figure 3 displays the ratios of standard deviations (SD) calculated from replicates from temperature uncompensated $\log S_{0}$ values, $S D(T)$, to those calculated from temperature normalized data, $S D\left(25{ }^{\circ} \mathrm{C}\right)$, plotted against the normalized SD. If the interlaboratory errors in $\log S_{0}$ measurement were attributable entirely to temperature effects, then the $\operatorname{SD}(\mathrm{T}) / \mathrm{SD}\left(25^{\circ} \mathrm{C}\right)$ ratio would greatly exceed unity. On the other hand, if there were large systematic errors in $\log S_{0}$ measurements not related to temperature, then the ratio would be near one.

When the Case C and D models were applied to the "4557-set" data [3], $94 \%$ of the calculated 
$\log S_{0}{ }^{r e f}-\log S_{0}^{\top}$ differences were between +0.2 and -0.2 . If the non-temperature related errors are substantially greater than 0.2 , then temperature normalization barely lowers interlaboratory errors. Figure 3 illustrates the impact of normalizing intrinsic solubility data to the $25^{\circ} \mathrm{C}$ benchmark temperature.

Figure 3a represents high-quality solubility measurements from the Yalkowsky et al. [8] handbook, albeit of relatively simple molecules. The interlaboratory errors are reduced 7.8 -fold on the average, to an average value $\mathrm{SD}\left(25^{\circ} \mathrm{C}\right)=0.06 \log$ unit.

Figure $3 \mathrm{~b}$ depicts a larger set of molecules, with a higher proportion of druglike molecules, which are less soluble than those in the case of Figure 3a. The interlaboratory errors are reduced 2.2-fold on the average, to an average value of $\mathrm{SD}\left(25^{\circ} \mathrm{C}\right)=0.17 \mathrm{log}$ unit. It is evident that interlaboratory errors not related to ionization and temperature effects are higher in the druglike set of molecules.

\section{Remaining Challenges in the Prediction of Enthalpy of Solution}

Table A1 also lists the calculated enthalpy values for the 55 molecules excluded from direct model construction. It is not a "validation" set, since many of the measurements were suspect, following the exclusion criteria. As it turns out, the calculated enthalpies of many of the excluded molecules are at comparable levels of agreement to the experimental enthalpy values. However, some compounds did show high variance. Mostly, these had reported negative enthalpies of solution, but the model calculated positive values (e.g., celecoxib, clotrimazole, droperidol, indinavir, ezetimibe, famotidine, fenofibrate, glibenclamide, loratidine, meloxicam, quetiapine, and saquinavir). Some excluded molecules had very high positive reported enthalpies, which the model only partly matched (triflupromazine, chlorpromazine, triflupromazine, etoxadrol, and rosiglitazone). It is suspected that data precision may be an underlying cause of the poor predictions. It is possible that some molecules may have been measured over a temperature range encompassing the Krafft or clouding points, which would have contributed to a highly nonlinear van't Hoff plot. Complications in solution chemistry, such as the formation of low-order aggregates, micelles, and complexes (with solution constituents) may contribute to increased errors [13-16]. For example, Pobudkowska et al. [25] found that aggregateforming chlorpromazine, triflupromazine, and trifluoperazine $\left(\mathrm{S}_{0} 0.5,1.1\right.$, and $2.7 \mu \mathrm{g} \mathrm{mL} \mathrm{m}^{-1}$, resp.) showed exceptionally high solubility temperature dependence, compared to more soluble (>10 $\mu \mathrm{g} \mathrm{mL}^{-1}$ ) phenothiazine derivatives. It would be helpful to measure the enthalpies of such molecules by direct calorimetry, to better understand the nature of the interlaboratory errors.

\section{Calculated Negative Enthalpies of Solution}

As listed in Table A1, there were 11 calculated negative values based on the Case C+D models, compared to 51 (non-excluded) negative measured values, with about half of these being about a standard deviation from zero. The average Abraham descriptors for the calculated negative enthalpy set were $0.9\left(\Sigma \alpha_{2}{ }^{\mathrm{H}}\right), 3.8\left(\Sigma \beta_{2}{ }^{\mathrm{H}}\right), 3.6$ $\left(\pi_{2}\right), 2.5\left(R_{2}\right)$, and $5.3\left(V_{x}\right)$. Given the MLR coefficients, the negative contributions were equally from $H$-bond basicity and the McGowan volume (-65 $\mathrm{kJ} \mathrm{mol}^{-1}$ contribution), but these were somewhat offset by positive contributions from dipolarity and molar refractivity ( $+40 \mathrm{~kJ} \mathrm{~mol}^{-1}$ contribution), resulting in a net negative calculated average enthalpy $\left(-17 \mathrm{~kJ} \mathrm{~mol}^{-1}\right)$. The RPT decision tree indicates that eight non-acids with $\mathrm{H}$-bond basicity exceeding $3.8 \mathrm{had}$ an average enthalpy of $-24 \pm 11 \mathrm{~kJ} \mathrm{~mol}^{-1}$ (node N01, Figure 1). There were 40 additional compounds with near zero enthalpy (node N000 in Figure 1). 

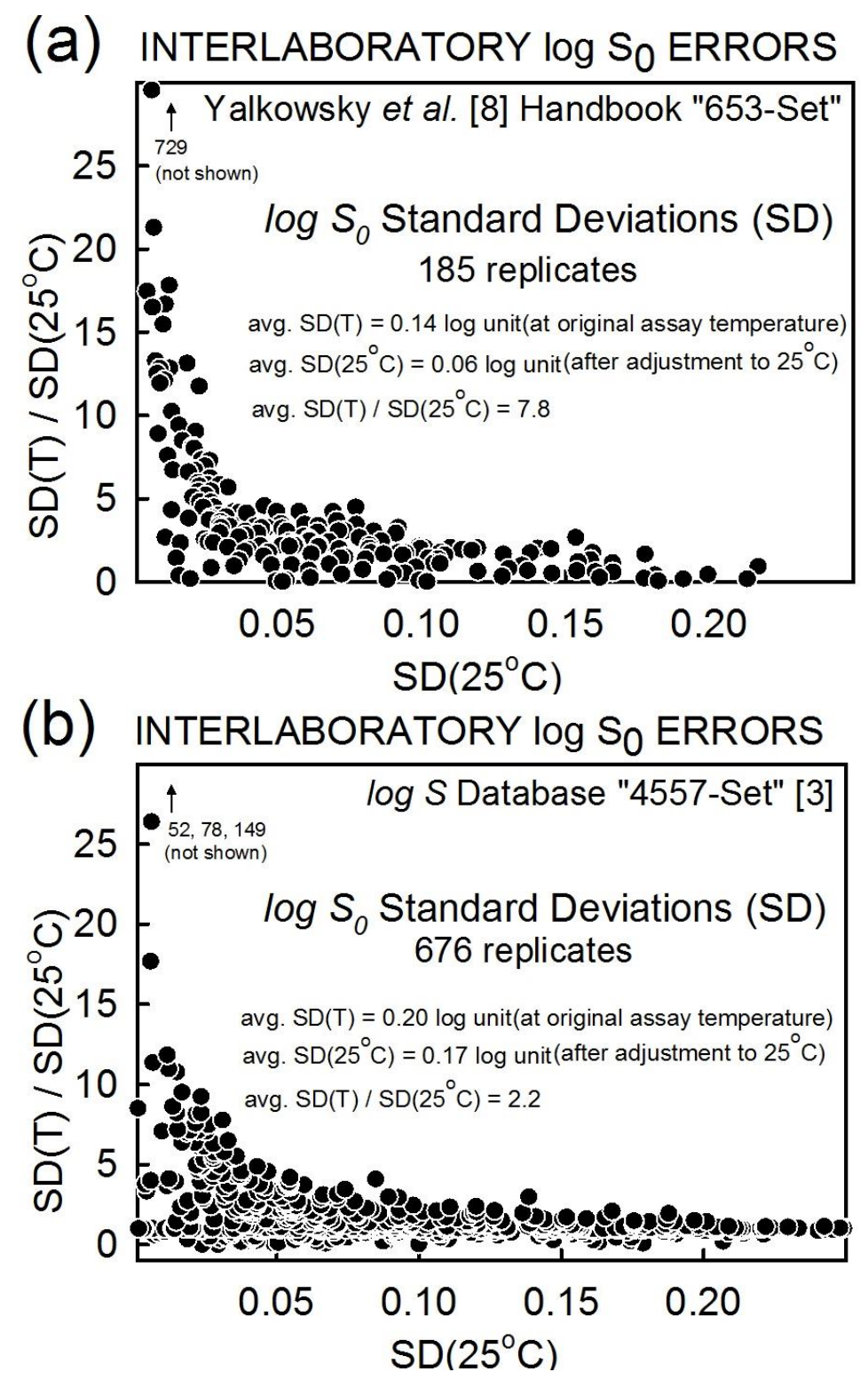

Figure 3. Standard deviations in $\log S_{0}$ determined in replicates at multiple temperatures, $S D(T)$, divided by those from $\log S_{0}$ transformed to the reference temperature, $\operatorname{SD}\left(25^{\circ} \mathrm{C}\right)$, as a function of $\operatorname{SD}\left(25^{\circ} \mathrm{C}\right)$. (a) High-quality solubility measurements from the Yalkowsky et al. [8] handbook, for relatively simple molecules. (b) The SD ratios for the entire "4557" set containing a large number of druglike molecules.

\section{MLR Model Validation}

The developed MLR model was validated by the leave-many-out (LMO) method, using the Algorithm Builder V1.8 program. In the approach, $20 \%$ of the dependent variables were randomly removed, with the MLR repeated 100 times, produced the $q^{2}=0.27$, with the $q^{2}$ standard deviation of 0.07 . These values are only slightly less than the value of $r^{2}(0.31)$ determined by normal MLR analysis.

\section{Conclusion}

The three different prediction methods tested (RFR, RPT, MLR) all indicated that the error in the enthalpy of solution data are $11-15 \mathrm{~kJ} \mathrm{~mol}^{-1}$, which is similar to estimates calculated from propagation-of-errors in 
solubility measurements $\left(\sim 10 \mathrm{~kJ} \mathrm{~mol}^{-1}\right)$ and with estimates based on interlaboratory replicates $\left(\sim 7 \mathrm{~kJ} \mathrm{~mol}^{-1}\right)$. According to the combined MLR model (Cases $C+D$ or E, Table 2 ), higher values of $m p, H$-bond acidity $\left(\sum \alpha_{2}{ }^{H}\right)$, polarizability/dipolarity $\left(\pi_{2}\right)$, and dispersion forces $\left(R_{2}\right)$ lead to more positive enthalpy values. Furthermore, big molecules (large $V_{x}$ ) that have high $\mathrm{H}$-bond basicity $\left(\Sigma \beta_{2}{ }^{\mathrm{H}}\right)$ are associated with more negative enthalpies of solution.

The main objective of this study was to demonstrate that the interlaboratory errors in solubility data mining can be lowered if solubility data are normalized for temperature effects. Before adjusting for ionization and temperature effects, the expected errors are commonly thought to be $0.5-0.7$ (or greater) log unit [4-7]. After transforming $S_{\mathrm{w}}$ to $S_{0}$ values (adjusting for ionization), it was estimated that temperature normalizations could lower the interlaboratory errors to near 0.15 log unit [3]. In the current study, we have shown this to be the case. Thus, for simple and moderately soluble molecules, the average interlaboratory errors are estimated to be about 0.06 log unit, lower than the 0.17 value estimated for sparingly-soluble druglike molecules. Consequently, the commonly cited interlaboratory solubility error of $0.5-0.7$ log unit in data mining warrants revision. It is thus expected that improved data quality will spur improved in silico prediction of solubility of druglike molecules.

\section{Acknowledgements}

We are grateful to Michael Abraham (University College London) and William E. Acree, Jr. (Univ. of North Texas) for pointing out many solubility-temperature and calorimetric enthalpy of solution publications. Helpful manuscript suggestions by Michael Abraham and Krisztina Takács-Novák (Semmelweis University, Budapest) are much appreciated.

\section{Glossary of Terms}

MLR multiple linear regression

RDK RDKit generated descriptors

RFR random forest regression method

RPT recursive partition tree analysis

$I_{A}, I_{B}, I_{A B}, I_{N}$ acid-base indicator indices: unit value, indicating that a molecule is an acid, base, ampholyte, or neutral, respectively, and zero otherwise

$\Sigma \alpha_{2}{ }^{H} \quad$ Abraham descriptor - solute $\mathrm{H}$-bond total acidity (also called A)

$\Sigma \beta_{2}{ }^{\mathrm{H}} \quad$ Abraham descriptor - solute $\mathrm{H}$-bond total basicity (also called B)

$\pi_{2} \quad$ Abraham descriptor - solute polarity/polarizability due to solute-solvent interactions between bond dipoles and induced dipoles (also called S)

$\mathrm{R}_{2} \quad$ Abraham descriptor - excess molar refraction $\left(\mathrm{dm}^{3} \mathrm{~mol}^{-1} / 10\right)$; which models dispersion force interaction arising from pi- and n-electrons of the solute (also called $\mathrm{E}$ )

$V_{x} \quad$ Abraham descriptor - McGowan molar volume $\left(\mathrm{dm}^{3} \mathrm{~mol}^{-1} / 100\right)$ of the solute

$\Sigma \alpha_{2}{ }^{H} \cdot \Sigma \beta_{2}{ }^{H}$ Abraham acid-base $\mathrm{H}$-bonding product descriptor [12]

$S \quad$ solubility, ideally expressed in units of $\mathrm{mol} / \mathrm{L}(\mathrm{M}), \mu \mathrm{g} / \mathrm{mL}$, or $\mathrm{mg} / \mathrm{mL}$

$S_{0} \quad$ "intrinsic" solubility (i.e., the solubility of the uncharged form of the compound)

$S_{w} \quad$ "water" solubility, defined by dissolving enough pure free acid/base (not drug salt) in distilled water (or water containing an inert salt - as ionic strength adjustor) to form a saturated solution. The final pH 
of the suspension, $\mathrm{pH}_{\text {sat }}$, and $\mathrm{S}_{0}$ can be calculated by the Henderson-Hasselbalch equation (when valid), provided the true $\mathrm{pK}_{\mathrm{a}}$ is known.

$S_{\mathrm{pH}} \quad$ "pH buffer" solubility (i.e., the total solubility of the compound at a well-defined measured $\mathrm{pH}$ )

$\Delta H_{\text {sol }}{ }^{0}$ change in enthalpy of solution at $25^{\circ} \mathrm{C}$ for uncharged molecules $\left(\Delta H_{\text {sol }}\right.$ implies the saturated-solution reference state, rather than the one based on unit activity)

\section{References}

[1] A. Avdeef, Adv. Drug Deliv. Rev. 59 (2007) 568-590

[2] A. Avdeef, Absorption and Drug Development, $2^{\text {nd }}$ Ed., Wiley-Interscience: Hoboken, NJ, 2012.

[3] A. Avdeef, ADMET \& DMPK 3(2) (2015) 84-109.

[4] A.R. Katritzky, Y. Wang, S. Sild, T. Tamm, M. Karelson, J. Chem. Inf. Comput. Sci. 38 (1998) 720-725.

[5] J. Taskinen, U. Norinder, In: B. Testa B, H. van de Waterbeemd (Eds.). Comprehensive Medicinal Chemistry II, Elsevier: Oxford, UK, 2007, pp. 627-648.

[6] W.L. Jorgensen, E.M. Duffy, Adv. Drug Deliv. Rev. 54 (2002) 355-366.

[7] D.S. Palmer, J.B.O. Mitchell, Mol. Pharmaceutics 11 (2014) 2962-2972.

[8] S.H. Yalkowsky, Y. He, P. Jain, Handbook of Aqueous Solubility Data, $2^{\text {nd }}$ Ed., CRC Press, Boca Raton, FL, 2010.

[9] G. Ismailos, C. Reppas, J. Dressman, P. Macheras, J. Pharm. Pharmacol. 43 (1991) 287-289.

[10] N. Sun, A. Avdeef, J. Pharm. Biomed. Anal. 56 (2011) 173-182.

[11] M.H. Abraham, A. Ibrahim, A.M. Zissimos, Y.H. Zhao, J. Comer, D.P. Reynolds, Drug Disc. Today 7 (2002) 1056-1063.

[12] M.H. Abraham, J. Le, J. Pharm. Sci. 88 (1999) 868-880.

[13] G. Völgyi, A. Marosi, K. Takács-Novák, A. Avdeef, ADMET \& DMPK 1(4) (2013) 48-62.

[14] A. Avdeef, ADMET \& DMPK 2(1) (2014) 33-42.

[15] A. Avdeef, ADMET \& DMPK 2(1) (2014) 43-55.

[16] G. Butcher, J. Comer, A. Avdeef, ADMET \& DMPK 3(2) (2015), 131-140.

[17] W.P. Walters, In: Bajorath J. (Ed.). Chemoinformatics for Drug Discovery. John Wiley \& Sons, Hoboken, NJ, 2014, pp. 1-31.

[18] G. Landrum, R. Lewis, A. Palmer, N. Stiefl, A. Vulpetti, J. Cheminform. 3 (2011) 1-1.

[19] A.S.I.D. Lang, J.-C. Bradley, ONS Melting Point Model 010. QDB archive DOI: 10.15152/QDB.104. QsarDB content. Property $\mathrm{mpC}$.

[20] V. Ruusmann, S. Sild, U. Maran, J. Chemoinform. 7 (2015) 32 (DOI: 10.1186/s13321-015-0082-6)

[21] U.S. Environmental Protection Agency. Molecular Descriptors Guide Description of the Molecular Descriptors Appearing in the Toxicity Estimation Software Tool, v.1.0.2 (2008).

[22] S.A. Wildman, G.M. Crippen, J. Chem. Inf. Comput. Sci. 39 (1999) 868-873.

[23] L.H. Hall, L.B. Kier, Comput. Chem. 2 (1991) 367-422.

[24] S.H. Bertz, J. Am. Chem. Soc. 103 (1981) 3599-3601.

[25] A. Pobudkowska, C. Ràfols, X. Subirats, E. Bosch, A. Avdeef, Eur. J. Pharm. Sci. - submitted Nov 2015.

[26] M. Donbrow, P. Sax, J. Pharm. Pharmaco. 34 (1982) 215-224.

[27] S.V. Kurkov, G.L. Perlovich, W. Zielenkiewics, J. Therm. Anal. Calorim. 83 (2006) 549-556.

[28] S. O. Nilsson, I. Wadsö, J. Chem. Thermodyn. 18 (1986) 673-681. 
[29] N. Nichols, R. Sköld, C. Spink, I. Wadsö, J. Chem. Thermodyn. 8 (1976) 993-999.

[30] T. Higuchi, FM. Shih, T. Kimura, J.H. Rytting, J. Pharm. Sci. 68 (1979) 1267-1272.

[31] S.H. Yalkowsky, S.C. Valvani, T.J. Roseman, J. Pharm. Sci. 72 (1983) 866-870.

[32] S.H. Yalkowsky, R.M. Dannenfelser, Aquasol Database of Aqueous Solubility, v5, College of Pharmacy, Univ. of Ariz, Tucson, AZ (1992).

[33] L. Pogliani, J. Chem. Inf. Comput. Sci. 36 (1996) 1082-1091.

[34] R. Kuhne, R.-U. Ebert, F. Kleint, G. Schmidt, G. Schuurmann, Chemosphere 30 (1995) 2061-2077.

[35] J. Dragun, C.S. Helling, Soil Sci. 139 (1985) 100-111.

[36] S.H. Yalkowsky, S.C. Valvani, J. Pharm. Sci. 69 (1980) 912-922.

[37] P.S. Winnek, C.L.A. Schmidt, J. Gen. Physiol. 18 (1935) 899-903.

[38] F.M. Plakogiannis, C. Iordanides, C. Siakali-Kiolafa, Drug Dev. Ind. Pharm. 6 (1980) 61-75.

[39] P.G. Wightman, J.B. Fein, Applied Geochem. 14 (1999) 319-331.

[40] K. Yoshida, T. Shigeoka, F. Yamauchi, Chemosphere 16 (1987) 2531-2544.

[41] G. Öjelund, R. Sköld, I. Wadsö. J. Chem. Thermodyn. 8 (1976) 45-54.

[42] J.N. Spencer, S.W. Barton, K.A. Smith, W.S. Wolbach, J.F. Powell, M.R. Kirshenbaum, D.W. Firth, Can. J. Chem. 61 (1983) 194-196.

[43] B. Schröder, L.M.N.B.F. Santos, I.M. Marrucho, J.A.P. Coutinho, Fluid Phase Equilib. 289 (2010) 140-147.

[44] A. Apelblat, E. Manzurola, J. Chem. Thermodyn. 21 (1989) 1005-1008.

[45] A. Apelblat, E. Manzurola, J. Chem. Thermodyn. 29 (1997) 1527-1533.

[46] W.E. May, S.P. Wasik, D.H. Freeman. Anal. Chem. 50 (1978) 175-179.

[47] W.E. May, S.P. Wasik, Anal. Chem. 50 (1978) 997-1000.

[48] W.E. May, S.P. Wasik, M.M. Miller, Y.B. Tewari, J.M. Brown-Thomas, R.N. Goldberg, J. Chem. Eng. Data 28 (1983) 197-200.

[49] P. Dohányosová, V. Dohnal, D. Fenclová. Fluid Phase Equilib. 214 (2003) 151-167.

[50] P.M. Gross, J.H. Saylor, J. Amer. Chem. Soc. 53 (1931) 1744-1751.

[51] H.A. Sober (Ed.), Handbook of Biochemistry, 2nd Ed., CRC Press: Cleveland,OH (1970) pp. B65-B68.

[52] F.L. Nordström, Å.C. Rasmuson, Eur. J. Pharm. Sci. 28 (2006) 377-384.

[53] J. Qing-Zhu, M. Pei-Sheng, Z. Huan, X. Shu-Qian, W. Qiang, Q. Yan, Fluid Phase Equilib. 250 (2006) 165172.

[54] E. Manzurola, A. Apelblat, J. Chem. Thermodyn. 34 (2002) 1127-1136.

[55] R.H. Manzo, A.A. Ahumada, J. Pharm. Sci. 79 (1990) 1109-1115.

[56] A.N. Paruta, Drug Dev. Ind. Pharm. 10 (1984) 453-465.

[57] S.H. Yalkowsky, G.L. Flynn, T.G. Slunick, J. Pharm. Sci. 61 (1972) 852-857.

[58] W.H. Streng, H.G.H. Tan, Int. J. Pharm. 25 (1985) 135-145.

[59] N. Nichols, I. Wadsö, J. Chem. Thermodyn. 7 (1975) 329-336.

[60] D. Todd, R.A. Winnike, Abstr. 9th Ann. Mtng., Amer. Assoc. Pharm. Sci., San Diego (1994).

[61] L. Vidaud, C. Kugel, G. Boccardi, S. Schmidt, J.-Y. Pommier, Int. J. Pharm. 437 (2012) 137-155.

[62] A.M. Zaichikov, N.G. Manin, Russ. J. Gen. Chem. 73 (2003) 1043-1050.

[63] J.W. Mullin, Crystallisation. Butterworths, London (1994), pp. 425-426.

[64] J.E. Fairbrother, Anal. Profiles Drug Subst. 3 (1974) 1-110.

[65] D.J.W. Grant, M. Mehdizadeh, A.H.-L. Chou, J.E. Fairbrother, Int. J. Pharm. 18 (1984), 25-38. 
[66] F.L. Mota, A.P. Carneiro, A.J. Queimada, S.P. Pinhob, E.A. Macedo, Eur. J. Pharm. Sci. 37 (2009) 499-507.

[67] S.D. Mithani, V. Bakatselou, C.N. TenHoor, J.B. Dressman, Pharm. Res. 13 (1996) 163-167.

[68] E. Rytting, K.A. Lentz, X.Q. Chen, F. Qian, S. Venkatesh, Pharm. Res. 21 (2004) 237-244.

[69] W.-H. Hong, T. Chang, R.E. Daly, Anal. Profiles Drug Subst. 15 (1986) 647-672.

[70] J. O'Neil, A. Smith, P.E. Heckelman (Eds.), The Merck Index. An Encyclodepia of Chemicals and Biologicals. 13th Ed., Merck \& Co. Inc., Whithouse Station, NJ, 2001.

[71] T.S. Logan, J. Amer. Chem. Soc. 67 (1945) 1182-1184.

[72] N.V. Sidgwick, J.A. Neill, J. Chem. Soc. (London) 123 (1923) 2813-2819.

[73] A.F. Fioritto, S.N. Bhattachar, J.A. Wesley, Int. J. Pharm. 330 (2007) 105-113.

[74] J. Parasrampuria, V. Das Gupta, J. Pharm. Sci. 79 (1990) 835-836.

[75] S. Haznedar, B. Dortunç, Int. J. Pharm. 269 (2004) 131-140.

[76] Y.P. Koparkar, V.G. Gaikar, J. Chem. Eng. Data 49 (2004) 800-803.

[77] W.R. Angus, R.P. Owen, J. Chem. Soc. (London) (1943) 231-232.

[78] R.A. Herman, P. Veng-Pedersen, J. Pharm. Sci. 83 (1994) 423-428.

[79] C.A.S. Bergström, U. Norinder, K. Luthman, P. Artursson, Pharm. Res. 19 (2002) 182-188.

[80] R. Carta, J. Chem. Eng. Data 44 (1999) 563-567.

[81] J.B. Dressman, A. Nair, B. Abrahamsson, D.M. Barends, D.W. Groot, S. Kopp, P. Langguth, J.E. Polli, V.P. Shah, M. Zimmer, J. Pharm. Sci. 101 (2012) 2653-2667.

[82] N. Ni, T. Sanghvi, S.H. Yalkowsky, Pharm. Res. 19 (2002) 1862-1866.

[83] H.A. Garekani, F. Sadeghi, A. Ghazi, Drug Dev. Ind. Pharm. 29 (2003) 173-179.

[84] M.A. Etman, V.F. Naggar, Ind. J. Pharm. 58 (1990) 177-184.

[85] M.P. Summers, R.P. Enever, J.E. Carless, Symp. Particle Growth Susp. (1972) 247-259.

[86] C.A.S. Bergström, M. Strafford, L. Lazarova, A. Avdeef, K. Luthman, P. Artursson, J. Med. Chem. 46 (2003) 558-570.

[87] P. Matsson, C.A.S. Bergström, N. Nagahara, S. Tavelin, U. Norinder, P. Artursson. J. Med. Chem. 48 (2005) 604-613.

[88] A.H. Shojaei, B. Berner, L. Xiaoling, Pharm. Res. 15 (1998) 1182-1188.

[89] A. Kristl, G. Vesnaver, A. Mrhar, F. Kozjek, Pharmazie 48 (1993) 608-610.

[90] J.N. Spenser, T.A. Judge, J. Solut. Chem. 12 (1983) 847-853.

[91] A. Apelblat. J. Chem. Thermodyn. 18 (1986) 351-357.

[92] A. Apelblat, E. Manzurola, J. Chem. Thermodyn. 19 (1987) 317-320.

[93] E.C. Attane, T.F. Doumani, Ind. Eng. Chem.41 (1949) 2015-2017.

[94] C. Zhong, Q. Hu, J. Pharm. Sci. 92 (2003) 2284-2294.

[95] A.A. Pradhan, J.H. Vera, Fluid Phase Equil. 152 (1998) 121-132.

[96] H.-C. Tseng, C.-Y. Lee, W.-L. Weng, I.-M. Shiah, Fluid Phase Equilib. 285 (2009) 90-95.

[97] L.A. Ferreira, E.A. Macedo, S.P. Pinho, Fluid Phase Equilib. 255 (2007) 131-137.

[98] H.B. Bull, K. Breese, C.A. Swenson, Biopolymers 17 (1978) 1091-1100.

[99] A. Glomme, J. März, J.B. Dressman, J. Pharm. Sci. 94 (2005) 1-16.

[100] A. Avdeef, M. Kansy, S. Bendels, K. Tsinman, Eur. J. Pharm. Sci. 33 (2008) 29-41.

[101] B.I. Escher, C. Berger, N. Bramaz, J.-H. Kwon, M. Richter, O. Tsinman, A. Avdeef, Envir. Tox. Chem. 27 (2008) 909-918.

[102] A. Fini, M. Laus, I. Orienti, V. Zecchi, J. Pharm. Sci. 75 (1986) 23-25. 
[103] C. Treiner, C. Vaution, G.N. Cave, J. Pharm. Pharmacol. 34 (1982) 539-540.

[104] D.L. Ross, C.M. Riley, Int. J. Pharm. 63 (1990) 237-250.

[105] F. Crea, D. Cucinotta, C. De Stefano, D. Milea, Sammartano S, Vianelli G. Eur. J. Pharm. Sci. 47 (2012) 661-677.

[106] M. Santana, M.P.A. Ribeiro, G.A. Leitte, R.L.C. Giordano, R.C. Giordano, S. Mattedi, AlChE J. 56 (2010) 1578-1583.

[107] V.B. Kurochkina, P.S. Nys, Chem. Pharm. J. (Russia) 37 (2003) 30-37.

[108] P.S. Nys, L.M. Elizarovskaya, N.N. Shellenberg, E.M. Savitskaya, Antibiotics 24 (1979) 895-902.

[109] E. Mentasti, C. Rinaudo, R. Boistelle, J. Chem. Eng. Data 28 (1983) 247-251.

[110] Y. Morimoto, T. Hatanaka, K. Sugibayashi, H. Omiya, J. Pharm. Pharmacol. 44 (1992) 634-639.

[111] A. Tsuji, E. Nakashima, S. Hamano, T. Yamana, J. Pharm. Sci. 67 (1978) 1059-1066.

[112] V.B. Kurochkina, A.V. Sklyarenko, J.E. Satarova, S.V. Yarotsky, Bioprocess Biosyst. Eng. 34 (2011) 11031117.

[113] A. Tsuji, E. Nakashima, T. Yamana, J. Pharmacol. Sci. 68 (1979) 308-311.

[114] J.P. Hou, J.W. Poole, J. Pharm. Sci. 58 (1969) 1510-1515.

[115] P.J. Sinko, Martin's Physical Pharmacy and Pharmaceutical Sciences. 5th Ed., Lippincott Williams \& Wilkins: Baltimore, MD, 2006, p. 250.

[116] G.M. Jacobsohn, D. Levenberg, Steriods Int. J. (San Francisco) 4 (1964) 849-853.

[117] M.A.Q. Chaudry, K.C. James, J. Med. Chem. 17 (1974) 157-161.

[118] J. Reza, A. Trejo, L.E. Vera-Avila, Chemosphere 47 (2002) 933-945.

[119] Y. Morimoto, R. Hori, T. Arita, Chem. Pharm. Bull. 22 (1974) 2217-2222.

[120] M. Xiao, W. Yan, Z. Zhang, J. Chem. Eng. Data 55 (2010) 3346-3348.

[121] X.Z. Jin, K.C. Chao, J. Chem. Eng. Data 37 (1992) 199-203.

[122] K. Hayashi, T. Matsuda, T. Takeyama, T. Hino, Agric. Biol. Chem. 30 (1966) 378-384.

[123] J.H. Fagerberg, E. Karlsson, J. Ulander, G. Hanisch, C.A.S. Bergström. Pharm. Res. 32 (2014) 578-589.

[124] E. Nicolaides, E. Galia, C. Efthymiopoulos, J.B. Dressman, C. Reppas, Pharm. Res. 16 (1999) 1877-1883.

[125] T.M. Ward, J.B. Weber, J. Agr. Food Chem. 16 (1968) 959-961.

[126] A. Apelblat, E. Manzurola, J. Chem. Thermodyn. 22 (1990) 289-292.

[127] K. Florey, Anal. Profiles Drug Subst. 17 (1988) 1-40.

[128] H. Nogami, T. Nagai, E. Fukuoka, T. Yotsuyanagi, Chem. Pharm. Bull. 17 (1969) 23-31.

[129] K. Sekiguchi, M. Kanke, N. Nakamura, Y. Tsuda, Chem. Pharm. Bull. 23 (1975) 1347-1352.

[130] J.C. Jespersen, K.T. Larsen, Yakugaku Zasshi 8 (1934) 212-226.

[131] A. Apelblat, E. Manzurola, N.A. Balal, J. Chem. Thermodyn. 38 (2006) 565-571.

[132] S. Korman, V.K. La Mer, J. Amer. Chem. Soc. 58 (1936) 1396-1403.

[133] G.L. Perlovich, N.N. Strakhova, V.P. Kazachenko, T.V. Volkova, V.V. Tkachev, K.-J. Schaper, O.A. Raevsky, Int. J. Pharm. 349 (2008) 300-313.

[134] G.L. Perlovich, N.N. Strakhova, V.P. Kazachenko, T.V. Volkova, V.V. Tkachev, K.-J. Schaper, O.A. Raevsky, Int. J. Pharm. 334 (2007) 115-124.

[135] J.C. Philip, R.S. Colborne, J. Chem. Soc. (London) 125 (1924) 492-500.

[136] J.H. Dolinski, Berich. Deut. Chem. Gesell. 38 (1905) 1835-1837.

[137] A. Avdeef, C.M. Berger, Eur. J. Pharm. Sci. 14 (2001) 281-291.

[138] K.G. Mooney, M.A. Mintun, K.J. Himmelstein, V.J. Stella, J. Pharm. Sci. 70 (1981) 70, 13-22. 
[139] K.J. Box, J.E.A. Comer, Curr. Drug Metab. 9 (2008) 869-878.

[140] T. Loftsson, D. Hreinsdótitir, AAPS PharmSciTech 7 (2006), Article 4, E1-E4.

[141] A. Giacalone, Gazz. Chim. Ital. 65 (1935) 844-850.

[142] L. Lu, X. Lu, J. Chem. Eng. Data 53 (2008) 1996-1998.

[143] K. Linderstrom-Lang, Comp. Rend. Trav. Lab. Carlsbreg 15 (1924) 15, 4-28.

[144] N. Meyyappan, N.N. Gandhi, J. Chem. Eng. Data 50 (2005) 796-800.

[145] S.K. Battu, M.A. Repka, S. Maddineni, A.G. Chittiboyina, M.A. Avery, S. Majumdar, AAPS PharmSciTech 11 (2010) 1466-1476.

[146] A. Glomme, J. März, J.B. Dressman. In: B. Testa, S.D. Krämer, H. Wunderli-Allenspach, G. Folkers (eds), Pharmacokinetic Profiling in Drug Research. Wiley-VCH, 2006, pp 259-280.

[147] H. Tomida, T. Yotsuyanagi, K. Ikeda, Chem Pharm Bull. (Tokyo) 26 (1978) 2832-2837.

[148] L. Zhou, L. Yang, S. Tilton, J. Wang, J. Pharm. Sci. 96 (2007) 3052-3071.

[149] S. Miyazaki, M. Oshiba, T. Nadai, Int. J. Pharm. 6 (1980) 77-85.

[150] C.A.S. Bergström, K. Luthman, P. Artursson, Eur. J. Pharm. Sci. 22 (2004) 387-398.

[151] J.C. Shah, M. Maniarb, J. Control. Release 23 (1993) 261-270.

[152] T. Suzuki, J. Comp.-Aided Molec. Design 5 (1991) 149-166.

[153] F.L. Mota, A.J. Queimada, S.P. Pinho, E.A. Macedo, Ind. Eng. Chem.Res. 47 (2008) 5182-5189.

[154] H. Matsuura, K. Sekiguchi, Yakuzaigaku 20 (1960) 213-218.

[155] E. Oliveri-Mandala, L. Irrera, Gazz. Chim. Ital. 60 (1930) 872-877.

[156] S. Kunadharaju, M. Savva, J. Chem. Eng. Data 55 (2010) 103-112.

[157] S. Rahman, D. Patel, M. Savva, AAPS PharmSciTech 15 (2014) 223-229.

[158] B. Faller, P. Ertl, Adv. Drug Deliv. Rev. 59 (2007) 533-545.

[159] J. Alsenz, M. Kansy, Adv. Drug Del. Rev. 59 (2007) 546-567.

[160] C. Sköld, S. Winiwarter, J. Wernevik, F. Bergström, L. Engström, R. Allen, K. Box, J. Comer, J. Mole, A. Hallberg, H. Lennernäs, T. Lundstedt, A.-L. Ungell, A. Karlén, J. Med. Chem. 49 (2006) 6660-6671.

[161] B. Bard, S. Martel, P.-A. Carrupt, Eur. J. Pharm. Sci. 33 (2008) 230-240.

[162] S. Clarysse, J. Browuwers, J. Tack, P. Annaert, P. Augustinjns, Eur. J. Pharm. Sci. 43 (2011) 260-269.

[163] X.-Q. Chen, S. Venkatesh, Pharm. Res. 21 (2004) 1758-1761.

[164] A. Alhalaweh, L. Roy, N. Rodríguez-Hornedo, S.P. Velaga, Mol. Pharmaceutics 9 (2012)2605?2612.

[165] J. Blanchard, J.O. Boyle, S. van Wagenen, J. Pharm. Sci. 77 (1988) 548-552.

[166] H. Burrows, M. Miguel, A. Varela, R. Becker, Thermchim. Acta 279 (1996) 77-82.

[167] T. Loftsson, S.B. Vogensen, C. Desbos, P. Jansook, AAPS PharmSciTech 9 (2008) 425-430.

[168] K. Beattie, G. Phadke, J. Novakovic, Profiles Drug Subst. Excip. Relat. Method. 38 (2013) 113-157.

[169] Y.-L. Hsieh, G.A. Ilevbare, B. van Eerdenbrugh, K.J. Box, M.V. Sanchez-Felix, L.S. Taylor, Pharm. Res. 29 (2012) 2738-2753.

[170] N.N. Efremov, Bull. Acad. Sci. USSR Div. Chem. Sci. (1940) 1-29.

[171] E. Shoghi, E. Fuguet, E. Bosch, C. Rafols, Eur. J. Pharm. Sci. 48 (2012) 290-300.

[172] S.D. Roy, G.L. Flynn, Pharm. Res. 5 (1988) 580-586.

[173] A. Tsuji, E. Nakashima, K. Nishide, Y. Deguchi, S. Hamano, T. Yamana, Chem. Pharm. Bull. 31 (1983) 40574069.

[174] Y. Li, F.-A. Wang, B.-Z. Ren, J. Chem. Eng. Data 55 (2010) 5375-5381.

[175] D. Zhang, Y. Wang, S. Ma, S. Wu, H. Hao, J. Chem. Eng. Data 58 (2013) 176-182. 
[176] N. Seedher, S. Bhatia, AAPS PharmSciTech 4 (2003) article 33, 1-9.

[177] L.P. Marrelli, Anal. Profiles Drug Subst. 4 (1975) 21-46.

[178] S. Wang, J. Wang, Q. Yin, Ind. Eng. Chem. Res. 44 (2005) 3783-3787.

[179] J.E. Fairbrother, Anal. Profiles Drug Subst. 2 (1973) 85-144.

[180] D. Szulczewski, F. Eng, Anal. Profiles Drug Subst. 4 (1975) 47-90.

[181] P.E. Macheras, M.E. Koupparis, S.A. Antimisiaris, J. Pharm. Sci. 78 (1989) 933-936.

[182] A.L. Green, J. Pharm. Pharmacol. 19 (1967) 10-16.

[183] H. Ogata, N. Aoyagi, N. Kaniwa, T. Shibazaki, E. Ejima, Y. Takagashi, T. Ogura, K. Tomita, S. Inoue, M. Zaizen, Int. J. Pharm. 23 (1985) 277-288.

[184] G. Schill, Acta Pharm. Suec. 2 (1965) 99-108.

[185] K.J. Box, G. Völgyi, E. Baka, M. Stuart, K. Takács-Novák, J. Pharm. Sci. 95 (2006) 1298-1307.

[186] A. Llinàs, R.C. Glen, J.M. Goodman, J. Chem. Inf. Model. 48 (2008) 1289-1303.

[187] A.P. ljzerman, Pharm. Res. 5 (1988) 772-775.

[188] H.M. Fadda, X. Chen, A. Aburub, D. Mishra, R. Pinal, Pharm. Res. 31 (2014) 1735-1743.

[189] C.M. Wassvik, A.G. Holmén, C.A.S. Bergström, I. Zamora, P. Artursson, Eur. J. Pharm. Sci. 29 (2006) 294305.

[190] A. Fini, A. Roda, R. Fugazza, B. Grigolo, J. Sol. Chem. 14 (1985) 595-603.

[191] G.L. Amidon, H. Lennernäs, V.P. Shah, J.R. Crison, Pharm. Res. 12 (1995) 413-420.

[192] S.S. Doosaj, W.V. Bhagwat, J. Ind. Chem. Soc. 10 (1933) 225-232.

[193] J. Meyer, Z. Elektrochem. 17 (1911) 976-984.

[194] S.R. Johnson, X.-Q. Chen, D. Murphy, O. Gudmundsson, Mol. Pharmaceutics 4 (2007) 513-523.

[195] X. Yu, G.L. Zipp, G.W.R. Davidson, III, Pharm. Res. 11 (1994) 522-527.

[196] M.E. Olivera, R.H. Manzo, H.E. Junginger, K.K. Midha, V.P. Shah, S. Stavchansky, J.B. Dressman, D.M. Barends, J. Pharm. Sci. 100 (2011) 22-33.

[197] Perry's Chemical Engineers' Handbook. 8th Ed. McGraw-Hill, New York (1997).

[198] Y. Nakagawa, S. Itai, T. Yoshida, T. Nagai, Chem. Pharm. Bull. 40 (1992) 725-728.

[199] A. Avdeef, S. Bendels, O. Tsinman, M. Kansy, Pharm. Res. 24 (2007) 530-545.

[200] V. Borhade, S. Pathak, S. Sharma, V. Patravale, Int. J. Pharm. 431 (2012) 138-148.

[201] J. Chung, F. Alvarez-Nunez, V. Chow, D. Daurio, J. Davis, M. Dodds, M. Emery, K. Litwiler, A. Paccaly, J. Peng, B. Rock, L. Wienkers, C. Yang, Z. Yu, J. Wahlstrom, J. Pharm. Sci. 104 (2015) 104, 1522-1532.

[202] J.N. Rakshit, Analyst (London) 46 (1921) 481-492.

[203] C.M. Wassvik, A.G. Holmén, R. Draheim, P. Artursson, C.A. Bergström, J. Med. Chem. 51 (2008) 30353039.

[204] N. Jain, S.H. Yalkowsky, J. Pharm. Sci. 90 (2001) 234-252.

[205] G. Ottaviani, S. Wendelspiess, R. Alvarez-Sanchez, Mol. Pharmaceutics 12 (2015) 1171-1179.

[206] M. De Groote, Amer. Perfum. (APRFA) 15 (1920) 372-374.

[207] R.K. Freier, Aqueous Solutions Volume I: Data for Inorganic and Organic Compounds. Walter deGruyter, New York (1976).

[208] J.W. Poole, C.K. Bahal, J. Pharm. Sci. 59 (1970) 1265-1267.

[209] R. Pinal, S.H. Yalkowsky, J. Pharm. Sci. 76 (1987) 75-85.

[210] S. Hovorka, A.H. Roux, G. Roux-Desgranges, V. Dohnal, J. Chem. Eng. Data 47 (2002) 954-959.

[211] G. Ismailos, C. Reppas, J. Dressman, P. Macheras, J. Pharm. Pharmacol. 43 (1991) 287-289. 
[212] G. Nan, J. Shi, Y. Huang, J. Sun, J. Lv, G. Yang, Y. Li, J. Chem. Eng. Data 59 (2014) 1304-1311.

[213] V. Bakatselou, R.C. Oppenheim, J.B. Dressman, Pharm. Res. 8 (1991) 1461-1469.

[214] L.-A. Erlich, D. Yu, D.A. Pallister, R.S. Levinson, D.G. Gole, P.A. Wilkinson, R.E. Erlich, L.E. Reeve, T.X. Viegas, Int. J. Pharm. 179 (1999) 49-53.

[215] M. Abbasi, F. Martinez, A. Jouyban, J. Molec. Liquids 197 (2014) 171-175.

[216] A.C. Kumoro, D.S. Retnowati, C.S. Budiyati, J. Chem. Eng. Data 55 (2010) 2603-2606.

[217] Y.B. Tewari, P.D. Gery, M.D. Vaudin, R.N. Glodberg, J. Chem. Thermodyn. 37 (2005) 233-241.

[218] E.M. Cohen, Anal. Profiles Drug Subst. 2 (1973) 163-198.

[219] B.W. Barry, D.I.D. El Eini, J. Pharm. Pharmacol. 28 (1976) 210-218.

[220] H.H. Lerner, Anal. Profiles Drug Subst. 4 (1975) 137-167.

[221] L.B. Pfendt, D.M. Sladić, T.J. Janjić, G. Popović, Analyst 115 (1990) 383-387.

[222] B.T. Bowman, W.W. Sans, J. Env. Sci. Health, Ser B 20 (1985) 625-631.

[223] A. Avdeef, C.M. Berger, C. Brownell, Pharm. Res. 17 (2000) 85-89.

[224] M.T. Ledwidge, O.I. Corrigan, Int. J. Pharm. 174 (1998) 187-200.

[225] A. Fini, G. Fazio, G. Feroci, Int. J. Pharm. 126 (1995) 95-102.

[226] A. Pobudkowska, U. Domańska, Chem. Indust. Chem. Eng. Quarterly 20 (2014) 115-126.

[227] G. Ottaviani, D.J. Gosling, C. Patissier, S. Rodde, L. Zhou, B. Faller, Eur. J. Pharm. Sci. 41 (2010) 452-457.

[228] W. Riess, H. Stierlin, P. Degen, J.W. Faigle, A. Gerardin, J. Moppert, W. Theobald, J. Wagner, Scand. J. Rheumato. Suppl. 22 (1978) 17-29.

[229] B. Chuasuwan, V. Binjesoh, J.E. Polli, H. Zhang, G.L. Amidon, H.E. Junginger, K.K. Midha, V.P. Shah, S. Stavchansky, J.B. Dressman, D.M. Barends, J. Pharm. Sci. 98 (2009) 1206-1219.

[230] M.L. Manca, M. Zaru, G. Ennas, D. Valenti, C. Sinico, G. Loy, A.M. Fadda, AAPS PharmSciTech 6 (2005) E464-E472.

[231] Y. Maitani, M. Nakagaki, T. Nagai, Int. J. Pharm. 74 (1991) 105-116.

[232] G.L. Perlovich, A.O. Surov, L.K.R. Hansen, A. Bauer-Brandl, J. Pharm. Sci. 96 (2007) 1031-1042.

[233] M. Stuart, K. Box, Anal. Chem. 77 (2005) 983-990.

[234] A. Llinàs, J.C. Burley, K.J. Box, R.C. Glen, J.M. Goodman, J. Med. Chem. 50 (2007) 979-983.

[235] G.L. Flynn, R.W. Smith, S.H. Yalkowsky, Technical Report (1972). (Cited in Ref. 8, p.1313)

[236] G.L. Perlovich, S.V. Kurkov, A. Bauer-Brandl, Eur. J. Pharm. 27 (2006) 150-157.

[237] N.M. Najib, M.S. Suleiman, Int. J. Pharm. 57 (1989) 197-203.

[238] M.L. Cotton, R.A. Hux, Anal. Prof. Drug Subst. 14 (1985) 491-526.

[239] T. Tokumira, Pharm. Tech. Jpn. 16 (2000) 1839-1846.

[240] L. Molin, G. Dahlstrom, M.-I. Nilsson, L. Tekenbergs, Acta Pharm. Suec. 20 (1983) 129-144.

[241] J. Kendall, L.E. Harrison, Trans. Faraday Soc. 24 (1928) 588-596.

[242] J. Takano, T. Yauoka, S. Mitsuzawa, Nipp. Kagaku Kaishi (1982) 1830-1834.

[243] S. Pinsuwan, A. Li, S. Yalkowsky, J. Chem. Eng. Data 40 (1995) 623-626.

[244] E.S. Kostewicz, U. Brauns, R. Becker, J.B. Dressman, Pharm. Res. 19 (2002) 345-349.

[245] C.M. Avila, F. Martinez, J. Solut. Chem. 31 (2002) 975-985.

[246] A.J. Hopfinger, E.X. Esposito, A. Llinàs, R.C. Glen, J.M. Goodman, J. Chem. Inf. Model. 49 (2009) 1-5.

[247] Beilstein Organischen Chemie, Berlin, Springer-Verlag. 4TH Ed. (Online: www.elsevier.com/onlinetools/reaxys). 
[248] S. Budavari, M.J. O'Neil, A. Smith, P.E. Heckelman, J.F. Kinneary, The Merck Index. An Encyclopedia of Chemicals and Drugs 12th Ed., Windholz M (Ed.) Rahway, NJ: Merck and Co., Inc. (1996).

[249] C.A. Janicki, R.K. Gilpin, Anal. Profiles Drug Subst. 7 (1978) 171-192.

[250] B. Kreilgård, Anal. Profiles Drug Subst. 6 (1977) 113-159.

[251] Y. Fukumori, T. Fukuda, Y. Yamamoto, N. Sato, Chem. Pharm. Bull. 31 (1983) 4029-4039.

[252] B. Lundberg, Acta Pharm. Suec. 16 (1979) 151-159.

[253] A.R. Hurwitz, S.T. Liu, J. Pharm. Sci. 66 (1977) 624-627.

[254] J. Morvay, J. Gyöngyösi, L. Kiss, Acta Pharm. Hung. 50 (1980) 243-247.

[255] G. Massol, F. Lamouroux, Comp. Rend. Hebdo. Sean. L'Acad. Sci. 128 (1899) 1000-1002.

[256] B. Chauhan, S. Shimpi, A. Paradkar, AAPS PharmSciTech 6 (2005) article 50, E405-E412.

[257] A. Mitra, F. Kesisoglou, P. Dogterom, AAPS PharmSciTech 16 (2014) 76-84.

[258] S.F. Kramer, G.L. Flynn, J. Pharm. Sci. 61 (1972) 1896-1904.

[259] J.C. Earle, J. Soc. Chem. Ind. London 37 (1918) 274-274.

[260] T. Taupitz, J.B. Dressman, S. Klein, Eur. J. Pharm. Biopharm. 84 (2013) 208-218.

[261] M.L.A.D. Lestari, F. Ardiana, G. Indrayanto, Profiles Drug Subst. Excip. Relat. Method. 36 (2011) 103-149.

[262] M.S. Islam, M.M. Narurkar, J. Pharm. Sci. 45 (1993) 682-686.

[263] N.M. Najib, M.S. Suleiman, Int. J. Pharm. 61 (1990) 173-178.

[264] C.A.S. Bergström, C.M. Wassvik, U. Norinder, K. Luthman, P. Artursson, J. Chem. Inf. Comput. Sci. 44 (2004) 1477-1488.

[265] S.A. Raina, B. van Eerdenbrugh, D.E. Alonzo, H. Mo, G.G.Z. Zhang, Y. Gao, L.S. Taylor, J. Pharm. Sci. 104 (2015), 1981-1992.

[266] S.V. Kurkov, G.L. Perlovich, Int. J. Pharm. 357 (2008) 100-107.

[267] P. Buch, P. Langguth, M. Kataoka, S. Yamashita, J. Pharm. Sci. 98 (2009) 2001-2009.

[268] S. Jamzad, R. Fassihi, AAPS PharmSciTech 7 (2006) article 33, E1-E6.

[269] T. Zimmermann, R.A. Yeates, H. Laufen, G. Pfaff, A. Wildfeuer, Eur. J. Clin. Pharmacol. 46 (1994) 147-150.

[270] A.K. Dash, W.F. Elmquist, Anal. Profiles Drug Subst. 27 (2001) 67-113.

[271] A. Mekkawy, M. Fathy, S. El-Shanawany, Br. J. Pharm. Res. 3 (2013) 293-313.

[272] N. Charoo, R. Cristofoletti, A. Graham, P. Laretey, B. Abrahamsson, D.W. Groot, S. Kopp, P. Langguth, J. Polli, V.P. Shah, J. Dressman, J. Pharm. Sci. 103 (2014) 3843-3858.

[273] C.D. Herzfeldt, R. Kuemmel, Drug Dev. Ind. Pharm. 9 (1983) 767-793.

[274] H. Terada, S. Muraoka, T. Fujita, J. Med. Chem. 17 (1974) 330-334.

[275] E. Abignente, P. de Caprariis, Anal. Profiles Drug Subst. 11 (1982) 313-343.

[276] B.D. Anderson, R.A. Conradi, J. Pharm. Sci. 74 (1985) 815-820.

[277] P. Li, L. Zhao, J. Pharm. Sci. 92 (2003) 951-956.

[278] S. Chandran, A. Roy, R.N. Saha, AAPS PharmSciTech 9 (2008) 1031-1037.

[279] Z. Wu, X. Li, C. Hou, Y. Qian, J. Chem. Eng. Data 55 (2010) 3958-3961.

[280] E. Baka, J.E.A. Comer, K. Takács-Novák, J. Pharm. Biomed. Anal. 46 (2008) 335-341.

[281] G.E. Granero, M.R. Longhi, M.J. Mora, H.E. Junginger, K.K. Midha, V.P. Shah, S. Savchansky, J.B. Dressman, D.M. Barends, J. Pharm. Sci. 99 (2010) 2544-2556.

[282] B. Davarakonda, D.P. Otto, A. Judefeind, R.A. Hill, M.M. De Villiers, Int. J. Pharm. 345 (2007) 142-153.

[283] K. Takács-Novák, V. Sz?ke, G. Völgyi, P. Horváth, R. Ambrus, P. Szabó-Révész, J. Pharm. Biomed. Anal. 83 (2013) 279-285. 
[284] P. Hajdu, A. Häussler, Arzneim-Forsch. 14 (1964) 709-710.

[285] M. Roseman, W.P. Jencks, J. Amer. Chem. Soc. 97 (1975) 631-640.

[286] L. Lu, X. Lu, J. Chem. Eng. Data 52 (2007) 37-39.

[287] A. Noubigh, C. Jeribi, A. Mgaidi, M. Abderrabba, J. Chem. Thermodynam. 55 (2012) 75-78.

[288] H. Wei, R. Löbenberg, Eur. J. Pharm. Sci. 29 (2006) 45-52.

[289] L.Y.S. Narasimham, V.D. Barhate, J. Pharmacy Res. 4 (2011) 532-536.

[290] P. Hadju, K.F. Kohler, F.H. Schmidt, H. Spingler, Arzneim.-Forsch. 19 (1969) 1381-1386.

[291] S. Grbić, J. Parojčić, S. Ibrić, Z. Djurić, AAPS PharmSciTech 12 (2011) 165-171

[292] S. Grbić, J. Parojčić, A. Malenović, Z. Djurić, M. Maksimović, J. Chem. Eng. Data 55 (2010) 1368-1371.

[293] W.S. Wise, E.B. Nicholson, J. Chem. Soc. (London) (1955) 2714-2716.

[294] E. Shafter, T. Higuchi, J. Pharm. Sci. 52 (1963) 781-791.

[295] T.R. Bates, M. Gibaldi, J.L. Kanig, J. Pharm. Sci. 55 (1966) 191-199.

[296] T.E. Needham, Jr., A.N. Paruta, R.J. Gerraughty, J. Pharm. Sci. 60 (1971) 565-567.

[297] R. Carta, G. Tola, J. Chem. Eng. Data 41 (1996) 414-417.

[298] X. Yang, X. Wang, C.B. Ching, J. Chem. Eng. Data 53 (2008) 1133-1137.

[299] J. Lu, X.J. Wang, X. Yang, C.B. Ching, J. Chem. Eng. Data 51 (2006)1593-1596.

[300] R. Takano, K. Sugano, A. Higashida, Y. Hayashi, M. Machida, Y. Aso, S. Yamashida, Pharm. Res. 23 (2006) 1144-1156.

[301] S. Li, S. Wong, S. Sethia, H. Almoazen, Y.M. Joshi, A.T.M. Serajuddin, Pharm. Res. 22 (2005) 628-635.

[302] H. Borsook, J.W. Dubnoff, J. Biol. Chem. 132 (1940) 307-324.

[303] H.P. Deppeler, Anal. Profiles Drug Subst. 10 (1981) 405-441.

[304] K.A. Levis, M.E. Lane, O.I. Corrigan, Int. J. Pharm. 253 (2003) 49-59.

[305] B.E. Mitchell, P.C. Jurs, J. Chem. Inf. Comput. Sci. 38 (1998) 489-496.

[306] H. Potthast, J.B. Dressman, H.E. Junginger, K.K. Midha, H. Oeser, V.P. Shah, H. Vogelpoel, D.M. Barends, J. Pharm. Sci. 94 (2005) 2121-2131.

[307] E. Stippler, Thesis: ISBN 3-8322-3218-4. Shaker Verlag, Aachen, Germany (2004).

[308] T.J. Difeo, J.E. Shuster, Anal. Profiles Drug Subst. 23 (1994) 229-268.

[309] B.D. Johnson, A. Howard, R. Varsolona, J. McCauley, D.K. Ellison, Anal. Profiles Drug Subst. Excip. 26 (1999) 319-357.

[310] B. Wuyts, J. Brouwers, R. Mols, J. Tack, P. Annaert, P. Augustijns, J. Pharm. Sci. 102 (2013) 3800-3807.

[311] J.H. Fagerberg, O. Tsinman, K. Tsinman, N. Sun, A. Avdeef, C.A.S. Bergström, Mol. Pharmaceutics 7 (2010) 1419-1430.

[312] K. Okimoto, R.A. Rajewski, K. Uekama, J.A. Jona, V.J. Stella, Pharm. Res. 13 (1996) 256-264.

[313] M.Z. Southard, D.W. Green, V.J. Stella, K.J. Himmelstein, Pharm. Res. 9 (1992) 58-69.

[314] A.K. Jain, Eur. J. Pharm. Biopharm. 68 (2008) 701-714.

[315] M. O’Brien, J. McCauley, E. Cohen, Anal. Profiles Drug Subst. 13 (1984) 211-235.

[316] Z. Ramtoola, O.I. Corrigan, Drug Dev. Ind. Pharm. 15 (1989) 2359-2374.

[317] E. Felder, M. Grandi, D. Pitré, G. Vittadini, Anal. Profiles Drug Subst. 17 (1988) 115-154.

[318] C. Becker, J.B. Dressman, G.L. Amidon, H.E. Junginger, S. Kopp, K.K. Midha, V.P. Shah, S. Stavchansky, D.M. Barends, J. Pharm. Sci. 96 (2007) 522-531.

[319] B.W. Long, L.S. Wang, J.S. Wu, J. Chem. Eng. Data 50 (2005) 136-137.

[320] D. Singh, K. Pathak, Int. J. Pharm. 441 (2013) 99-110. 
[321] F. Edwards, C. Tsakmaka, S. Mohr, P.R. Fielden, N.J. Goddard, J. Booth, K.Y. Tam, Analyst 138 (2013) 339345.

[322] A. Diakidou, M. Vertzoni, J. Dressman, C. Reppas, Biopharm. Drug Dispos. 30 (2009) 318-325.

[323] M. Vertzoni, A. Diakidou, M. Chatzilias, E. Söderlind, B. Abrahamsson, J. B. Dressman, C. Reppas, Pharm. Res. 27 (2010) 2187-2196.

[324] J.J. Sheng, N.A. Kasim, R. Chandrasekharan, G.L. Amidon, Eur. J. Pharm. Sci. 29 (2006) 306-314.

[325] Y.W. Alelyunas, J.R. Empfield, D. McCarthy, R.C. Spreen, K. Bui, L. Pelosi-Kilby, C. Shen, Bioorg. Med. Chem. Lett. 20 (2010) 7312-7316.

[326] R. Mashru, V. Sutariya, J. Sankalia, J. Pharm. Pharmacol. Sci. 8 (2005) 54-62.

[327] K. Beattie, G. Phadke, J. Novakovic, Profiles Drug Subst. Excip. Relat. Method. 37 (2012) 245-285.

[328] S. Soltanpour, W.E. Acree, Jr., A. Jouyban, J. Chem. Eng. Data 55 (2010) 570-573.

[329] M.F. Powell, Anal. Profiles Drug Subst. 15 (1986) 761-792.

[330] N.I. Nakano, J. Pharm. Sci. 68 (1979) 667-668.

[331] E. Shoghi, E. Fuguet, C. Ràfols, E. Bosch, Chem. Biodivers. 6 (2009) 1789-1795.

[332] G. Popović, M. Čakar, D. Agbaba, J. Pharm. Biomed. Anal. 49 (2009) 42-47.

[333] E.A. Klobbi, Zeit. Physik. Chem. (Lepzig) 14 (1894) 615-632.

[334] K. Daniel-Mwambete, S. Torrado, C. Cuesta-Bandera, F. Ponce-Gordo, J.J. Torrado, Int. J. Pharm. 272 (2004) 29-36.

[335] R.J. Allan, T.R. Watson, Eur. J. Drug Metab. Pharmacol. 8 (1983) 373-381.

[336] S. Charumanee, A. Titwan, J. Sirithunyalug, S. Okonogi, J. Chem. Tech. Biotech. 81 (2006) 523-529.

[337] J.A. Clements, S.D. Popli, Can. J. Pharm. Sci. 8 (1973) 88-92.

[338] D.L. French, J.W. Mauger, Pharm. Res. 10 (1993) 1285-1290.

[339] W.I. Higuchi, P.K. Lau, T. Higuchi, J.W. Shell, J. Pharm. Sci. 52 (1963) 150-153.

[340] A. Burger, Arszneimittel-Foreschung 25 (1975) 24-27.

[341] M.J. Cho, R.R. Kurtz, C. Lewis, S.M. Machkovech, D.J. Houser, J. Pharm. Sci. 71 (1982) 410-14.

[342] S.D. Roy, G.L. Flynn, Pharm. Res. 6 (1989) 147-151.

[343] L. Fouriea, J.C. Breytenbach, J.D. Plessisa, C. Goosen, H. Swart, J. Hadgraft, Int. J. Pharm. 279 (2004) 5966.

[344] T.S. Lakshmi, P.K. Nandi, J. Phys. Chem. 80 (1976) 249-252

[345] V.N. Kulakov, A.G. Artyukh, I.M. Nikiforov, T.V. Barinova, J. Appl. Chem. USSR 54 (1981) 335-338.

[346] Y.I. Korenman, E.I. Polumestnaya, Russ. J. Phys. Chem. 51 (1977) 1392-1392.

[347] M.P. Moyle, M. Tyner, Ind. Eng. Chem.45 (1953) 1794-1797.

[348] G.N. Pulley, Ind. Eng. Chem.Anal. Ed. 8 (1936) 360.

[349] R. Chadha, P. Arora, A. Saini, D.S. Jain, AAPS PharmSciTech 11 (2010) 1328-1339.

[350] L. Hertelendi, Zeits. Physik. Chem. A 192 (1943) 379-380.

[351] J. Hecq, M. Deleers, D. Fanara, H. Vranckx, K. Amighi, Int. J. Pharm. 299 (2005) 167-177.

[352] B. Davarakonda, R.A. Hill, M.M. De Villiers, Int. J. Pharm. 284 (2004) 133-140.

[353] J. Bres, F. Bressolle, M.T. Huguet, Trav. Soc. Pharm. Montpellier 36 (1976) 331-364.

[354] W. McBride, R.A. Henry, G.B.L. Smith, J. Amer. Chem. Soc. 71 (1949) 2937-2938.

[355] D.E. Cadwallader, H.W. Jun, Anal. Profiles Drug Subst. 5 (1976) 345-374.

[356] L.-K. Chen, D.E. Cadwallader, H.W. Jun, J. Pharm. Sci. 65 (1976) 868-872.

[357] E.F. McNiff, P.S.K. Yap, H.-L. Fung, Anal. Profiles Drug Subst. 9 (1980) 519-542. 
[358] G.A. Lewis, R.P. Enever, Int. J. Pharm. 3 (1979) 319-333.

[359] H. Koike, T. Konse, T. Sada, T. Ikeda, A. Hyogo, D. Hinman, H. Saito, H. Yanagisawa, Annu. Rep. Sankyo Res. Lab. 55 (2003) 1-91.

[360] L. Bajerski, R.C. Rossi, C.L. Dias, A.M. Bergold, P.E. Froechlich, AAPS PharmSciTech 11 (2010) 637-644

[361] G. Popović, D.M. Sladić, V.M. Stefanović, L.B. Pfendt, J. Pharm. Biomed. Anal. 31 (2003) 693-699.

[362] A.T.M. Serajuddin, M. Rosoff, J. Pharm. Sci. 73 (1984) 1203-1208.

[363] G. Völgyi, E. Baka, K.J. Box, J.E.A. Comer, K. Takács-Novák, Anal. Chim. Acta 673 (2010) 40-46.

[364] S. Miyazaki, H. Inouie, T. Nadai, T. Arita, M. Nakano, Chem. Pharm. Bull. 27 (1979) 1441-1447.

[365] N.J. Irwin, C.P. McCoy, D.S. Jones, S.P. Gorman, Pharm. Res. 30 (2013) 857-865.

[366] A.T.M. Serajuddin, D. Mufson, Pharm. Res. 2 (1985) 65-68.

[367] S. Nicoli, F. Zani, S. Bilzi, R. Bettini, P. Santi, Eur. J. Pharm. Biopharm. 69 (2008) 613-621.

[368] Y. Archand, J. Hawari, S.R. Guiott, Water Res. 29 (1995) 131-136.

[369] T.D. Wilson, Anal. Profiles Drug Subst. 13 (1984) 361-416.

[370] J. Burgess, R.I. Haines, J. Chem. Eng. Data 23 (1978) 196-197.

[371] Z. Yang, H. Hu, X. Zhang, Y. Xu, J. Chem. Eng. Data 52 (2007) 184-185.

[372] A.T.M. Serajuddin, C.I. Jarowski, J. Pharm. Sci. 74 (1985) 142-147.

[373] A. Avdeef, D. Voloboy, A. Foreman, In: Testa B, van de Waterbeemd H (eds.). Comprehensive Medicinal Chemistry II, Elsevier: Oxford, UK, 2007, pp. 399-423.

[374] Y. Kato, Y. Okamoto, S. Nagasawa, T. Ueki, Chem. Pharm. Bull. 29 (1981) 3410-3413.

[375] G.H. Parsons, C.H. Rochester, A. Rostron, P.C. Sykes, J. Phys. Chem. Soc. Perkin 2 (1972) 136-138.

[376] S. Ahmadian, V. Panahi-Azar, M.A.A. Fakhree, W.E. Acree, Jr., A. Jouyban, J. Chem. Eng. Data 56 (2011) 4352-4355.

[377] Y. Moroi, K. Sato, R. Matuura, J. Phys. Chem. 86 (1982) 2463-2468.

[378] C.T. Chiou, V.H. Freed, D.W. Schmedding, R.L. Kohnert, Environ. Sci. Technol. 11 (1977) 475-478.

[379] X. Zhou, J. Fan, N. Li, Z. Du, H. Ying, J. Wu, J. Xiong, J. Bai, Fluid Phase Equil. 316 (2012) 26-33.

[380] A.T.M. Serajuddin, C.I. Jarowski, J. Pharm. Sci. 82 (1993) 306-310.

[381] P.A. Schwartz, C.T. Rhodes, J.W. Cooper, Jr., J. Pharm. Sci. 66 (1977) 994-997.

[382] P.-C. Chiang, H. Wong, AAPS J. 15 (2013) 1109-1117.

[383] J. Philip, I.J. Holcomb, S.A. Fusari, Anal. Profiles Drug Subst. 13 (1984) 417-445.

[384] J. Jinno, D.-M. Oh, J.R. Crison, G.L. Amidon, J. Pharm. Sci. 89 (2000) 268-274.

[385] H. Okuyama, Y. Ikeda, S. Kasai, K. Imamori, K. Takayama, T. Nagai, Int. J. Pharm. 186 (1999) 141-148.

[386] W.L. Hayton, D.E. Guttman, G. Levy, J. Pharm. Sci. 61 (1972) 356-361.

[387] R.M.C. Dawson, D.C. Elliot, W.H. Elliot, K.M. Jones, Data Biochem. Res. 1,1, Oxford Univ. Press, Pergamon (1969).

[388] S.G. Frank, M.J. Cho, J. Pharm. Sci. 67 (1978) 1665-1668.

[389] T.J. Roseman, S.H. Yalkowsky, J. Pharm. Sci. 62 (1973) 1680-1685.

[390] L.-H. Wang, Y.-Y. Chang, J. Chem. Eng. Data 50 (2005) 1375-1376.

[391] C. Becker, J.B. Dressman, G.L. Amidon, H.E. Junginger, S. Kopp, K.K. Midha, V.P. Shah, S. Stavchansky, D.M. Barends, J. Pharm. Sci. 97 (2008) 3709-3720.

[392] J.P. Phillips, H.P. Price, J. Amer. Chem. Soc. 73 (1951) 4414-4415.

[393] W.A. Lee, L. Gu, A.R. Miksztal, P.H. Nelson, Pharm. Res. 7 (1990) 161-166.

[394] X. Sun, Y. Shao, W. Yan, J. Chem. Eng. Data 53 (2008) 2562-2566. 
[395] T.T. Mariappan, S. Singh, Int. J. Tuberc. Lung Dis. 7 (2003) 797-803.

[396] G. Boman, P. Lundgren, G. Stjernstrom, Eur. J. Clin. Pharmacol. 8 (1975) 293-299.

[397] S. Agrawal, R. Panchagnula, Int. J. Pharm. 287 (2004) 97-112.

[398] G.G. Gallo, P. Radaelli, Anal. Profiles Drug Subst. 5 (1976) 467-514.

[399] S. Baboota, M. Dhaliwal, K. Kohli, AAPS PharmSciTech 5 (2005) E83-E90.

[400] M.M. Kamila, N. Mondal, L.K. Ghosh, B.K. Gupta, AAPS PharmaSciTech 10 (2009) 887-899.

[401] D. Schönherr, U. Wollatz, D. Haznar-Garbacz, U. Hanke, K.J. Box, R. Taylor, R. Ruiz, S. Beato, D. Becker, W. Weitschies, Eur. J. Pharm. Biopharm. 92 (2015) 155-170.

[402] H.M.C. Marques, J. Hadgraft, I.W. Kellaway, Int. J. Pharm. 63 (1990) 259-266.

[403] M. Donbrow, E. Touitou, H. Ben-Shalom, J. Pharm. Pharmacol. 28 (1976) 766-769.

[404] A.T.M. Serajuddin, C.I. Jarowski, J. Pharm. Sci. 74 (1985) 148-154.

[405] J.D. Gaynor, V. Van Volk, J. Agric. Food Chem. 29 (1981) 1143-1146.

[406] T.-C. Bai, G.-B. Yan, J. Hu, C.-G. Huang, Int. J. Pharm. 308 (2006) 100-106.

[407] M. Mirmehrabi, S. Rohani, L. Perry, J. Pharm. Sci. 95 (2006) 798-809.

[408] W.F. Ng, C.F. Poe, J. Amer. Pharm. Assoc. (Sci. Ed.) 45 (1956) 351-353.

[409] S. Horiba, Memoirs of the College of Engineering, Kyoto Imperial Univ. 2 (1917) 519-519.

[410] G.A. Lewis, Int. J. Pharm. 18 (1984) 207-212.

[411] H.B. Kostenbauder, F.B. Gable, A.N. Martin, J. Am. Pharm. Assoc. (Sci. Ed.) 42 (1953) 210-213.

[412] F.S. Hom, J. Autian, J. Am. Pharm. Assoc. 45 (1956) 608-611.

[413] H.A. Krebs, J.C. Speakman, J. Chem. Soc. (1945) 593-595.

[414] H.A. Krebs, J.C. Speakman, Br. Med. J. (1946) 47-50.

[415] J.B. Ziegler, R.E. Bagdon, A.C. Shabica, Amer. J. Digest. Disord. 21 (1954) 74-77.

[416] H. Stober, W. DeWitte, Anal. Profiles Drug Subst. 11 (1982) 523-546.

[417] R.D.G. Wolfenden, Anal. Profiles Drug Subst. 6 (1977) 515-578.

[418] N.M. El-Guindi, F.M. Abdel-Gawad, J. Drug Res. Egypt 16 (1985) 185-192.

[419] R.H. Kienle, J.M. Sayward, J. Amer. Chem. Soc. 64 (1942) 2464-2468.

[420] J.K. Guillory, H.O. Lin, Chem. Pharm. Bull. 24 (1976) 1675-1678.

[421] M.L. Crossley, E.H. Northey, M.E. Hultquist, J. Amer. Chem. Soc. 62 (1940) 372-374.

[422] T. Higuchi, M. Gupta, L.W. Busse, J. Amer. Pharm. Assoc. (Sci. Ed.) 42 (1953) 157-161.

[423] V.K. Kapoor, Anal. Profiles Drug Subst. Excip. 22 (1993) 389-430.

[424] M. Kanke, K. Sekiguchi, Chem. Pharm. Bull. 21 (1973) 878-884.

[425] F.M. Plakogiannis, J.A. McCauley, Anal. Profiles Drug Subst. 13 (1984) 573-596.

[426] A.C. Rouw, G. Somen, J. Chem. Thermodyn. 13 (1981) 67-76.

[427] C. Goosen, T.J. Laing, J. du Plessis, T.C. Goosen, G.L. Flynn, Pharm. Res. 19 (2002) 13-19.

[428] S.H. Yalkowsky, S. Banerjee, In: Aqueous Solubility, Methods for Estimation for Organic Compounds. Marcel Dekker: New York, 1992, pp128-148.

[429] U. Avico, C. Signoretti, R. Di Francesco, E. Cingolani, Bull. Chim. Framaceutico 115 (1976) 242-253.

[430] A. Häussler, P. Hajdu, Archiv. Pharm. 291 (1958) 531-536.

[431] A.A. Forist, T. Chulski, Metab. Clin. Exp. 5 (1956) 807-812.

[432] D. Taylor, G.C. Vincent, J. Chem. Soc. (London) (1952) 3218-3224.

[433] C.R. Behl, L.H. Block, M.L. Borke, J. Pharm. Sci. 65 (1976) 429-430. 
[434] L.W. Dittert, T. Higuchi, D.R. Reese, J. Pharm. Sci. 53 (1964) 1325-1328.

[435] A. Post, R.J. Warren, J.E. Zarembo, Anal. Profiles Drug Subst. 9 (1980) 543-582.

[436] A.A. El-Harakany, A.O. Barakat, J. Sol. Chem. 14 (1985) 263-269.

[437] H. Nogami, T. Nagai, H. Uchida, Chem. Pharm. Bull. 16 (1968) 2257-2262.

[438] C.-Y. Lee, J.-T. Chen, W.-T. Chang, I.-M. Shiah, Fluid Phase Equilib. 343 (2013) 30-35.

[439] D.I. Hitchcock, J. Gen. Physiol. 6 (1924) 747-757.

[440] A. Seidell, Solubilities of Organic Compounds. New York: D. Van Norstrand Co. Inc. (1941). 


\section{Appendix}

Table A1 lists the molecules studied, along with the $\mathrm{mp}$ and ABSOLV descriptors.

Table A1. Enthalpy of Solution, Melting Points, and Abraham Solvation Descriptors

\begin{tabular}{|c|c|c|c|c|c|c|c|c|c|c|}
\hline Compound ${ }^{a}$ & Type ${ }^{b}$ & $\mid \begin{array}{l}\text { obs } \boldsymbol{\Delta} \boldsymbol{H}_{\text {sol }} \\
\mathrm{kJ} \mathrm{mol}^{-1}\end{array}$ & $\begin{array}{c}\text { Calc } \\
\Delta H_{\text {sol }} \\
\mathrm{kJ} \mathrm{mol}^{-1}\end{array}$ & $\mathrm{mp}^{\mathrm{c}}$ & $\begin{array}{l}\Sigma \alpha_{2}{ }^{H} \\
(A)\end{array}$ & $\begin{array}{c}\Sigma \beta_{2}{ }^{\mathrm{H}} \\
(\mathrm{B})\end{array}$ & $\pi(S)$ & $R_{\mathbf{2}}$ (E) & $\begin{array}{c}\text { Mc } \\
\text { Gowan } \\
\text { Vol. }\left(\mathbf{V}_{\mathbf{x}}\right)\end{array}$ & References $^{d}$ \\
\hline (2-naphthoxy)acetic acid & A & 56 & 29 & 156 & 0.57 & 0.72 & 1.56 & 1.51 & 1.50 & [26] \\
\hline [4-(benzyloxy)phenyl]acetic acid & A & 46 & 28 & 123 & 0.57 & 0.75 & 1.64 & 1.38 & 1.88 & [27] \\
\hline 1,1-bis(acetyloxy)propyl acetate & $\mathrm{N}$ & -4 & -5 & $\underline{31}$ & 0.00 & 1.07 & 1.32 & 0.17 & 1.60 & [28] \\
\hline 1,1-bis(butanoyloxy)propyl butanoate & $\mathrm{N}$ & -6 & -12 & $\underline{27}$ & 0.00 & 1.09 & 1.35 & 0.16 & 2.45 & [28] \\
\hline 1,1-bis(propanoyloxy)propyl propanoate & $\mathrm{N}$ & -7 & -9 & $\underline{30}$ & 0.00 & 1.08 & 1.34 & 0.17 & 2.02 & [28] \\
\hline 1,4-butanediol & $\mathrm{N}$ & -11 & 6 & 20 & 0.63 & 0.59 & 0.73 & 0.41 & 0.79 & [29] \\
\hline 1,4-diaminobutane & B & -37 & 4 & 27 & 0.42 & 1.12 & 0.82 & 0.40 & 0.87 & [29] \\
\hline $17 \alpha$-methyltestosterone & $\mathrm{N}$ & -13 & 13 & 164 & 0.31 & 1.02 & 2.22 & 1.53 & 2.52 & {$[30-32]$} \\
\hline 1-butyltheobromine (excluded ${ }^{* *}$ ) & $\mathrm{N}$ & 0 & 12 & 108 & 0.00 & 1.28 & 1.92 & 1.47 & 1.79 & {$[32,33]$} \\
\hline 1-naphthylamine (excluded ${ }^{* *}$ ) & B & 2 & 24 & 49 & 0.23 & 0.49 & 1.40 & 1.58 & 1.19 & {$[34,35]$} \\
\hline 2-benzoyloxy ethanol (excluded ${ }^{* *}$ ) & $\mathrm{N}$ & 7 & 9 & 78 & 0.23 & 0.82 & 1.25 & 0.88 & 1.27 & {$[31,36]$} \\
\hline 2,3-dichloro-L-tyrosine & $A B$ & 22 & 17 & $\underline{175}$ & 1.30 & 1.13 & 1.60 & 1.35 & 1.62 & [37] \\
\hline 2,3-quinolino-phthalide (Form I) & $A B$ & 49 & 27 & $\underline{208}$ & 0.13 & 1.15 & 2.12 & 2.48 & 2.10 & [38] \\
\hline 2,3-quinolino-phthalide (Form II) & $A B$ & 11 & 29 & $\underline{215}$ & 0.13 & 1.15 & 2.17 & 2.45 & 1.96 & [38] \\
\hline 2,3-quinolino-phthalide (Form III) & $A B$ & 13 & 23 & $\underline{203}$ & 0.13 & 1.07 & 1.65 & 1.90 & 1.63 & [38] \\
\hline 2,3-quinolino-phthalide (Form IV) & $A B$ & 16 & 24 & $\underline{206}$ & 0.13 & 1.07 & 1.71 & 1.88 & 1.49 & [38] \\
\hline 2,3-quinolino-phthalide (Form V) & $A B$ & 25 & 23 & $\underline{200}$ & 0.13 & 1.07 & 1.65 & 1.90 & 1.63 & [38] \\
\hline 2,4,6-trichlorophenol (excluded) & A & -28 & 26 & 67 & 0.42 & 0.15 & 0.94 & 1.07 & 1.14 & {$[39,40]$} \\
\hline 2-acetamido-N-methylacetamide & $\mathrm{N}$ & 2 & 11 & $\underline{117}$ & 0.51 & 1.15 & 1.92 & 0.62 & 1.04 & [41] \\
\hline 2-aminopyridine & B & 4 & 18 & 58 & 0.23 & 0.62 & 1.14 & 0.92 & 0.78 & [42] \\
\hline 2-aminopyrimidine & B & 4 & 21 & 126 & 0.23 & 0.70 & 1.18 & 0.96 & 0.73 & [42] \\
\hline 2-furoic acid & A & 39 & 26 & 134 & 0.57 & 0.48 & 0.95 & 0.53 & 0.75 & [43] \\
\hline 2-furoic acid & A & 40 & 26 & 134 & 0.57 & 0.48 & 0.95 & 0.53 & 0.75 & [44] \\
\hline 2-methoxybenzoic acid & A & 29 & 25 & 99 & 0.57 & 0.66 & 1.17 & 0.81 & 1.13 & [45] \\
\hline 2-methylanthracene & $\mathrm{N}$ & 46 & 28 & 206 & 0.00 & 0.23 & 1.28 & 2.02 & 1.60 & [46-48] \\
\hline 2-methylanthracene & $\mathrm{N}$ & 47 & 28 & 206 & 0.00 & 0.23 & 1.28 & 2.02 & 1.60 & {$[49]$} \\
\hline 2-nitroaniline (excluded ${ }^{* *}$ ) & $\mathrm{N}$ & -5 & 18 & 72 & 0.18 & 0.48 & 1.44 & 1.16 & 0.99 & {$[34,50]$} \\
\hline
\end{tabular}




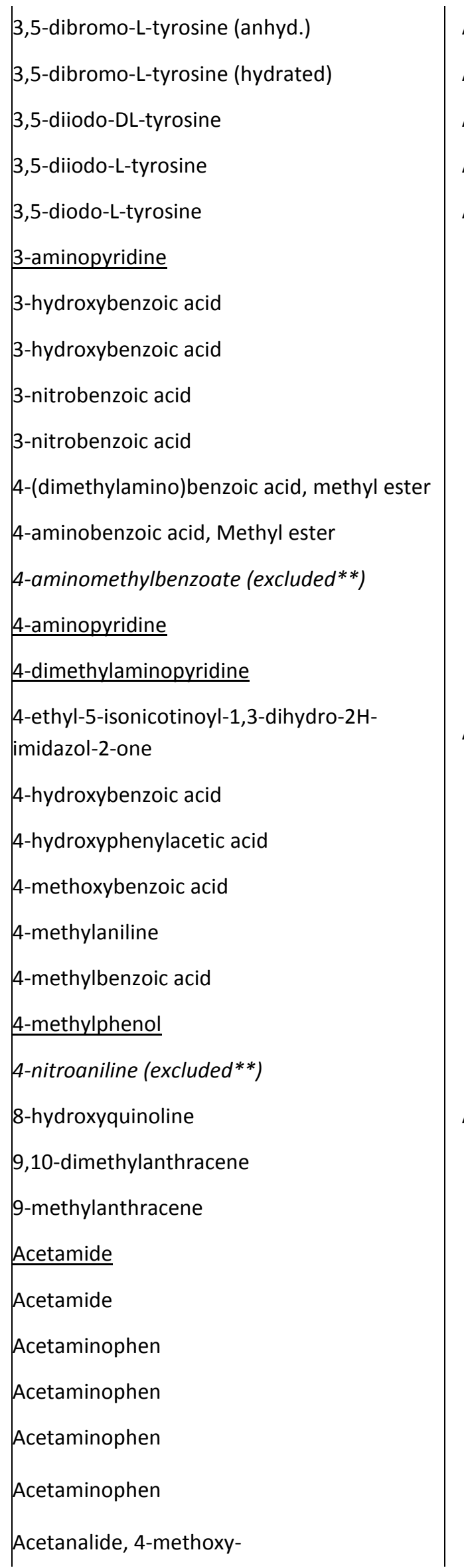

\begin{tabular}{|c|c|c|c|c|c|c|c|c|c|}
\hline$A B$ & 17 & 20 & $\underline{175}$ & 1.03 & 1.10 & 1.69 & 1.69 & 1.72 & [37] \\
\hline$A B$ & 28 & 20 & $\underline{175}$ & 1.03 & 1.10 & 1.69 & 1.69 & 1.72 & [37] \\
\hline$A B$ & 24 & 26 & 213 & 1.03 & 1.14 & 1.82 & 2.31 & 1.89 & [37] \\
\hline$A B$ & 33 & 26 & 200 & 1.03 & 1.14 & 1.82 & 2.31 & 1.89 & [37] \\
\hline$A B$ & 33 & 26 & 200 & 1.03 & 1.14 & 1.82 & 2.31 & 1.89 & [51] \\
\hline B & 3 & 18 & 61 & 0.23 & 0.71 & 1.21 & 0.90 & 0.78 & [42] \\
\hline A & 32 & 30 & 203 & 1.06 & 0.72 & 1.29 & 0.98 & 0.99 & [43] \\
\hline A & 33 & 30 & 203 & 1.06 & 0.72 & 1.29 & 0.98 & 0.99 & [52] \\
\hline A & 26 & 29 & 142 & 0.64 & 0.54 & 1.65 & 1.02 & 1.11 & {$[32,34,53,54]$} \\
\hline A & 28 & 29 & 142 & 0.64 & 0.54 & 1.65 & 1.02 & 1.11 & [43] \\
\hline B & 27 & 23 & 372 & 0.00 & 0.79 & 1.26 & 0.88 & 1.45 & [55] \\
\hline B & 28 & 18 & 112 & 0.23 & 0.76 & 1.42 & 0.95 & 1.17 & [56] \\
\hline B & 27 & 18 & 112 & 0.23 & 0.76 & 1.42 & 0.95 & 1.17 & {$[31,57]$} \\
\hline B & 4 & 21 & 159 & 0.23 & 0.71 & 1.21 & 0.90 & 0.78 & [42] \\
\hline B & 3 & 15 & 112 & 0.00 & 0.74 & 1.04 & 0.84 & 1.06 & [42] \\
\hline$A B$ & 16 & 22 & $\underline{189}$ & 0.36 & 1.48 & 2.27 & 1.57 & 1.60 & [58] \\
\hline A & 42 & 30 & 215 & 1.00 & 0.72 & 1.29 & 0.98 & 0.99 & {$[43,45]$} \\
\hline A & 40 & 28 & 150 & 1.07 & 0.72 & 1.29 & 0.98 & 1.13 & [43] \\
\hline A & 28 & 27 & 184 & 0.57 & 0.66 & 1.17 & 0.81 & 1.13 & [45] \\
\hline B & 1 & 17 & 44 & 0.23 & 0.43 & 1.02 & 0.88 & 0.96 & {$[32,34]$} \\
\hline A & 24 & 28 & 180 & 0.57 & 0.44 & 1.02 & 0.77 & 1.07 & [43] \\
\hline A & 3 & 24 & 34 & 0.50 & 0.39 & 0.85 & 0.81 & 0.92 & [59] \\
\hline B & 32 & 26 & 147 & 0.28 & 0.53 & 1.65 & 1.13 & 0.99 & {$[34,50]$} \\
\hline$A B$ & -4 & 20 & 76 & 0.07 & 0.73 & 1.36 & 1.55 & 1.10 & {$[32,60,61]$} \\
\hline $\mathrm{N}$ & 48 & 26 & 182 & 0.00 & 0.23 & 1.22 & 2.04 & 1.74 & [49] \\
\hline $\mathrm{N}$ & 42 & 23 & 75 & 0.00 & 0.23 & 1.28 & 2.02 & 1.60 & [49] \\
\hline $\mathrm{N}$ & 10 & 18 & 81 & 0.49 & 0.57 & 1.09 & 0.42 & 0.51 & [62] \\
\hline $\mathrm{N}$ & 6 & 13 & 222 & 0.49 & 0.57 & 1.09 & 0.42 & 0.51 & [63] \\
\hline A & 20 & 29 & 170 & 0.91 & 0.93 & 1.66 & 1.12 & 1.17 & [64] \\
\hline A & 23 & 29 & 170 & 0.91 & 0.93 & 1.66 & 1.12 & 1.17 & [65] \\
\hline A & 23 & 29 & 170 & 0.91 & 0.93 & 1.66 & 1.12 & 1.17 & [66] \\
\hline A & 29 & 29 & 170 & 0.91 & 0.93 & 1.66 & 1.12 & 1.17 & {$[32,67,68,83,84$} \\
\hline $\mathrm{N}$ & 26 & 24 & 400 & 0.41 & 0.87 & 1.55 & 0.96 & 1.31 & [55] \\
\hline
\end{tabular}




Acetanilide
Acetanilide
Acetanilide, 2-NH2-
Acetazolamide
Acetophenone, 4-HO-
Acetyl-r-mandelic acid
Acetylsalicylic acid
Acetylsalicylic acid
Acetylsalicylic acid (Form_I)
Acetylsalicylic acid (Form_II)
Acetylsalicylic acid (Form_IV)
Acyclovir
Aminopyine
Aminine (Form A)
Adipic acid
Adipic acid
Adipic acid
Adipic acid
Adipic acid, 3-Methyl-
Ajmaline
Alanine, D-
Alanine, DL-
Alaninine, DL-

\begin{tabular}{|c|c|c|c|c|c|c|c|c|c|}
\hline $\mathrm{N}$ & 18 & 15 & 114 & 0.41 & 0.67 & 1.42 & 0.89 & 1.11 & {$[31,32,70]$} \\
\hline $\mathrm{N}$ & 21 & 15 & 114 & 0.41 & 0.67 & 1.42 & 0.89 & 1.11 & [71] \\
\hline B & 32 & 22 & 135 & 0.65 & 0.94 & 1.76 & 1.22 & 1.21 & [72] \\
\hline A & 34 & 31 & 260 & 0.85 & 1.50 & 2.55 & 1.64 & 1.34 & {$[32,68,73-75]$} \\
\hline A & 39 & 27 & 110 & 0.66 & 0.70 & 1.34 & 1.02 & 1.07 & [76] \\
\hline A & 45 & 25 & 97 & 0.57 & 0.81 & 1.47 & 0.82 & 1.43 & [77] \\
\hline A & 23 & 26 & 142 & 0.57 & 0.77 & 1.42 & 0.84 & 1.29 & [43] \\
\hline A & 26 & 26 & 142 & 0.57 & 0.77 & 1.42 & 0.84 & 1.29 & {$[68,70,78-82]$} \\
\hline A & 38 & 26 & 135 & 0.57 & 0.77 & 1.42 & 0.84 & 1.29 & [85] \\
\hline A & 32 & 26 & 135 & 0.57 & 0.77 & 1.42 & 0.84 & 1.29 & [85] \\
\hline A & 28 & 26 & 135 & 0.57 & 0.77 & 1.42 & 0.84 & 1.29 & [85] \\
\hline$A B$ & 24 & 19 & 255 & 0.65 & 2.18 & 1.95 & 2.04 & 1.52 & {$[79,86-89]$} \\
\hline$A B$ & 24 & 36 & 363 & 0.60 & 0.98 & 1.79 & 1.74 & 0.92 & [90] \\
\hline A & 33 & 26 & 153 & 1.14 & 0.70 & 1.07 & 0.34 & 1.10 & [91] \\
\hline A & 34 & 26 & 153 & 1.14 & 0.70 & 1.07 & 0.34 & 1.10 & [92] \\
\hline A & 36 & 26 & 153 & 1.14 & 0.70 & 1.07 & 0.34 & 1.10 & [43] \\
\hline A & 38 & 26 & 153 & 1.14 & 0.70 & 1.07 & 0.34 & 1.10 & [65] \\
\hline A & 56 & 25 & 101 & 1.14 & 0.73 & 1.06 & 0.34 & 1.24 & [93] \\
\hline B & 8 & 20 & 206 & 0.63 & 1.68 & 1.87 & 2.49 & 2.46 & [8] \\
\hline AB & 8 & 16 & 291 & 0.78 & 0.93 & 0.92 & 0.38 & 0.71 & [51] \\
\hline AB & 9 & 14 & 264 & 0.78 & 0.93 & 0.92 & 0.38 & 0.71 & [51] \\
\hline$A B$ & 8 & 15 & 245 & 0.78 & 0.93 & 0.92 & 0.38 & 0.71 & [63] \\
\hline$A B$ & 11 & 9 & $\underline{112}$ & 0.78 & 0.93 & 0.92 & 0.38 & 0.71 & [94-97] \\
\hline$A B$ & 8 & 17 & 315 & 0.78 & 0.93 & 0.92 & 0.38 & 0.71 & [98] \\
\hline$A B$ & 8 & 16 & 291 & 0.78 & 0.93 & 0.92 & 0.38 & 0.71 & [51] \\
\hline$A B$ & 22 & 22 & $\underline{174}$ & 0.71 & 1.12 & 1.96 & 2.01 & 1.95 & [99-101] \\
\hline$A B$ & 5 & 4 & 151 & 1.19 & 1.82 & 1.26 & 1.43 & 1.98 & [8] \\
\hline A & 15 & 26 & 92 & 0.57 & 0.70 & 1.32 & 1.06 & 1.63 & [102] \\
\hline A & 26 & 26 & 171 & 0.52 & 1.32 & 1.47 & 1.22 & 1.57 & [103] \\
\hline A & 49 & 29 & 350 & 0.27 & 1.54 & 1.04 & 1.43 & 0.88 & [66] \\
\hline$A B$ & 22 & 13 & $\underline{198}$ & 0.71 & 2.34 & 2.58 & 2.18 & 2.37 & [104] \\
\hline$A B$ & 4 & 13 & 202 & 0.80 & 1.94 & 1.69 & 1.59 & 1.47 & [105-108] \\
\hline B & 7 & 12 & 135 & 0.00 & 1.79 & 1.88 & 1.56 & 1.87 & [119] \\
\hline B & 12 & 12 & 135 & 0.00 & 1.79 & 1.88 & 1.56 & 1.87 & {$[8,68,110]$} \\
\hline
\end{tabular}




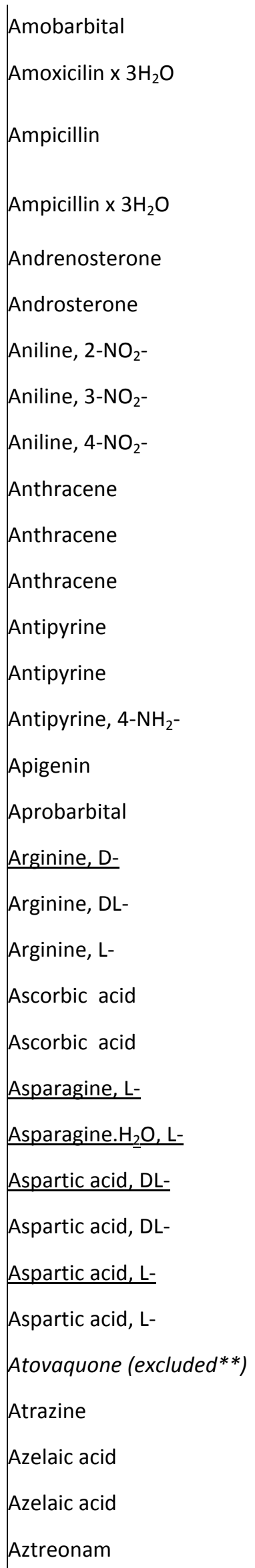

\begin{tabular}{|c|c|c|c|c|c|c|c|c|c|}
\hline A & 27 & 25 & 157 & 0.57 & 1.50 & 1.54 & 1.16 & 1.80 & [103] \\
\hline$A B$ & 19 & 15 & $\underline{196}$ & 1.55 & 2.90 & 3.22 & 2.70 & 2.54 & {$[86,105,111-113]$} \\
\hline$A B$ & 25 & 16 & 204 & 1.06 & 2.62 & 3.01 & 2.48 & 2.48 & $\begin{array}{c}{[8,105,106,111} \\
113,114]\end{array}$ \\
\hline$A B$ & 7 & 15 & 198 & 1.06 & 2.62 & 3.01 & 2.48 & 2.48 & {$[2,8,68,111,113]$} \\
\hline $\mathrm{N}$ & 25 & 21 & 220 & 0.00 & 1.31 & 2.99 & 1.77 & 2.36 & [116] \\
\hline $\mathrm{N}$ & 25 & 14 & 185 & 0.31 & 0.96 & 2.21 & 1.43 & 2.43 & [116] \\
\hline $\mathrm{N}$ & 36 & 18 & 72 & 0.18 & 0.48 & 1.44 & 1.16 & 0.99 & [117] \\
\hline B & 35 & 23 & 72 & 0.30 & 0.53 & 1.65 & 1.13 & 0.99 & [117] \\
\hline B & 37 & 23 & 72 & 0.28 & 0.53 & 1.65 & 1.13 & 0.99 & [117] \\
\hline $\mathrm{N}$ & 45 & 30 & 216 & 0.00 & 0.23 & 1.34 & 1.99 & 1.45 & [49] \\
\hline $\mathrm{N}$ & 47 & 30 & 216 & 0.00 & 0.23 & 1.34 & 1.99 & 1.45 & {$[46-48]$} \\
\hline $\mathrm{N}$ & 48 & 30 & 216 & 0.00 & 0.23 & 1.34 & 1.99 & 1.45 & [118] \\
\hline B & 20 & 17 & 114 & 0.00 & 1.28 & 1.75 & 1.42 & 1.49 & [119] \\
\hline B & 23 & 17 & 112 & 0.00 & 1.28 & 1.75 & 1.42 & 1.49 & {$[8,31,110]$} \\
\hline B & 25 & 15 & 109 & 0.21 & 1.72 & 1.91 & 1.65 & 1.58 & [119] \\
\hline A & 29 & 36 & 348 & 1.30 & 1.20 & 2.25 & 2.36 & 1.85 & [120] \\
\hline A & 21 & 25 & 141 & 0.52 & 1.29 & 1.40 & 1.11 & 1.61 & [103] \\
\hline$A B$ & 5 & 7 & 235 & 1.26 & 1.95 & 1.24 & 1.06 & 1.39 & [51] \\
\hline$A B$ & 18 & 7 & 230 & 1.26 & 1.95 & 1.24 & 1.06 & 1.39 & [121] \\
\hline$A B$ & 19 & 8 & 244 & 1.26 & 1.95 & 1.24 & 1.06 & 1.39 & [122] \\
\hline A & 22 & 25 & 187 & 1.12 & 1.84 & 1.93 & 1.42 & 1.11 & [43] \\
\hline A & 23 & 25 & 187 & 1.12 & 1.84 & 1.93 & 1.42 & 1.11 & [44] \\
\hline$A B$ & 24 & 17 & 235 & 1.27 & 1.48 & 1.84 & 0.80 & 0.96 & [51] \\
\hline$A B$ & 34 & 15 & 235 & 1.58 & 1.77 & 2.07 & 0.89 & 1.13 & [51] \\
\hline$A B$ & 29 & 15 & 270 & 1.18 & 1.26 & 1.37 & 0.55 & 0.92 & [51] \\
\hline$A B$ & 31 & 15 & 270 & 1.18 & 1.26 & 1.37 & 0.55 & 0.92 & [45] \\
\hline$A B$ & 24 & 14 & 230 & 1.18 & 1.26 & 1.37 & 0.55 & 0.92 & [51] \\
\hline$A B$ & 27 & 14 & 230 & 1.18 & 1.26 & 1.37 & 0.55 & 0.92 & [45] \\
\hline A & 8 & 34 & 224 & 0.31 & 1.21 & 2.54 & 2.49 & 2.69 & {$[123,124]$} \\
\hline B & 27 & 17 & 173 & 0.36 & 0.89 & 1.24 & 1.26 & 1.62 & {$[8,70,125]$} \\
\hline A & 38 & 25 & 104 & 1.14 & 0.71 & 1.09 & 0.33 & 1.53 & [126] \\
\hline A & 39 & 25 & 104 & 1.14 & 0.71 & 1.09 & 0.33 & 1.53 & [43] \\
\hline$A B$ & 33 & 18 & 227 & 0.84 & 2.66 & 3.62 & 2.46 & 2.76 & [127] \\
\hline
\end{tabular}




\begin{tabular}{|c|c|c|c|c|c|c|c|c|c|c|}
\hline Barbital (Form_l) & A & 19 & 26 & 190 & 0.52 & 1.21 & 1.35 & 0.98 & 1.37 & [128] \\
\hline Barbital (Form_II) & A & 17 & 26 & 190 & 0.52 & 1.21 & 1.35 & 0.98 & 1.37 & [129] \\
\hline Barbital (Form_III) & A & 30 & 26 & 190 & 0.52 & 1.21 & 1.35 & 0.98 & 1.37 & [128] \\
\hline Barbituric acid, 5,5-propyl-2- & A & 24 & 25 & 146 & 0.52 & 1.21 & 1.36 & 0.98 & 1.66 & [130] \\
\hline Barbituric acid, 5-allyl-5-buthyl- & A & 13 & 25 & $\underline{145}$ & 0.52 & 1.27 & 1.42 & 1.10 & 1.75 & [130] \\
\hline Barbituric acid, 5-allyl-5-phenyl- & A & 24 & 28 & 157 & 0.52 & 1.34 & 1.87 & 1.68 & 1.80 & [130] \\
\hline Barbituric acid, 5-Me-5-phenyl- & A & 25 & 29 & 226 & 0.52 & 1.29 & 1.80 & 1.56 & 1.56 & [130] \\
\hline Barbituric acid, i-Pr- & A & 24 & 27 & 215 & 0.52 & 1.23 & 1.38 & 1.02 & 1.23 & [130] \\
\hline Benzamide & $\mathrm{N}$ & 18 & 19 & 130 & 0.49 & 0.66 & 1.55 & 1.00 & 0.97 & [63] \\
\hline Benzenepentacarboxylic acid & A & 29 & 25 & $\underline{212}$ & 2.86 & 1.76 & 2.61 & 1.52 & 1.79 & [131] \\
\hline Benzenesulfonamide, 2- $\mathrm{NH}_{2}-$ & $A B$ & 34 & 21 & 155 & 0.67 & 1.18 & 1.94 & 1.44 & 1.20 & [132] \\
\hline Benzenesulfonamide, 3- $\mathrm{NH}_{2-}^{-}$ & $A B$ & 39 & 21 & 141 & 0.67 & 1.14 & 1.92 & 1.44 & 1.20 & [132] \\
\hline $\begin{array}{l}\text { Benzenesulfonamide, 4-amino-N-(2,3- } \\
\text { dichlorophenyl)- }\end{array}$ & $A B$ & 14 & 29 & $\underline{194}$ & 0.66 & 1.05 & 2.63 & 2.21 & 2.05 & [133] \\
\hline $\begin{array}{l}\text { Benzenesulfonamide, 4-amino-N-(2,5- } \\
\text { dichlorophenyl)- }\end{array}$ & $A B$ & 24 & 29 & $\underline{192}$ & 0.66 & 1.05 & 2.66 & 2.23 & 2.05 & [133] \\
\hline $\begin{array}{l}\text { Benzenesulfonamide, 4-amino-N-(3,4- } \\
\text { dichlorophenyl)- }\end{array}$ & $A B$ & 30 & 30 & $\underline{193}$ & 0.71 & 1.05 & 2.66 & 2.23 & 2.05 & [133] \\
\hline $\begin{array}{l}\text { Benzenesulfonamide, 4-amino-N-(4- } \\
\text { chlorophenyl)- }\end{array}$ & $A B$ & 38 & 28 & $\underline{185}$ & 0.64 & 1.12 & 2.59 & 2.12 & 1.93 & [133] \\
\hline Benzenesulfonamide, N-(2,3-dichlorophenyl)- & A & 28 & 31 & 114 & 0.43 & 0.74 & 2.21 & 1.90 & 1.95 & [134] \\
\hline Benzenesulfonamide, N-(2-chlorophenyl)- & A & 15 & 30 & 125 & 0.36 & 0.81 & 2.14 & 1.79 & 1.83 & [134] \\
\hline Benzenesulfonamide, N-(4-chlorophenyl)- & A & 38 & 30 & 122 & 0.42 & 0.81 & 2.17 & 1.82 & 1.83 & [134] \\
\hline Benzenesulfonic acid, 3- $\mathrm{NH}_{2}-$ (anhyd.) & $A B$ & 19 & 27 & 300 & 0.67 & 1.14 & 1.92 & 1.44 & 1.20 & {$[135]$} \\
\hline Benzenesulfonic acid, 4- $\mathrm{NH}_{2-}^{-}$ & $A B$ & 21 & 19 & 122 & 0.54 & 1.19 & 2.20 & 1.17 & 1.16 & {$[136]$} \\
\hline Benzocaine & B & 41 & 15 & 89 & 0.23 & 0.76 & 1.43 & 0.94 & 1.31 & {$[55]$} \\
\hline Benzocaine & B & 35 & 16 & 91 & 0.23 & 0.76 & 1.43 & 0.94 & 1.31 & {$[31,32,57,68,78]$} \\
\hline Benzoic acid & A & 26 & 26 & 122 & 0.57 & 0.44 & 1.08 & 0.75 & 0.93 & [43] \\
\hline Benzoic acid & A & 27 & 26 & 122 & 0.57 & 0.44 & 1.08 & 0.75 & 0.93 & {$[53]$} \\
\hline Benzoic acid & A & 28 & 26 & 122 & 0.57 & 0.44 & 1.08 & 0.75 & 0.93 & {$[131]$} \\
\hline Benzoic acid & A & 35 & 26 & 122 & 0.57 & 0.44 & 1.08 & 0.75 & 0.93 & $\begin{array}{c}{[32,53,68,137-} \\
140]\end{array}$ \\
\hline Benzoic acid, 2- $\mathrm{NO}_{2}-$ & A & 25 & 29 & 146 & 0.57 & 0.54 & 1.65 & 1.02 & 1.11 & [53] \\
\hline Benzoic acid, 3-Me-6- $\mathrm{NO}_{2}-$ & A & 39 & 28 & 135 & 0.57 & 0.54 & 1.59 & 1.04 & 1.25 & {$[141]$} \\
\hline Benzoic acid, 3- $\mathrm{NO}_{2-}^{-}$ & A & 31 & 29 & 141 & 0.64 & 0.54 & 1.65 & 1.02 & 1.11 & [53] \\
\hline
\end{tabular}




\begin{tabular}{|c|c|c|c|c|c|c|c|c|c|c|}
\hline Benzoic acid, 4-HO- & A & 33 & 30 & 216 & 1.00 & 0.72 & 1.29 & 0.98 & 0.99 & [53] \\
\hline Benzophenone, 2,2',4,4'-(HO) $4^{-}$ & A & 45 & 34 & 201 & 1.63 & 0.98 & 2.11 & 2.13 & 1.72 & [142] \\
\hline Benzophenone, 2,3,4-( $\mathrm{HO})_{3^{-}}$ & A & 45 & 32 & 141 & 1.02 & 0.76 & 1.97 & 1.84 & 1.66 & [142] \\
\hline Benzophenone, 2,4-(HO) $)_{2}^{-}$ & A & 28 & 31 & 145 & 0.80 & 0.68 & 1.81 & 1.73 & 1.60 & [142] \\
\hline Benzoquinone & $\mathrm{N}$ & 21 & 10 & 116 & 0.00 & 0.76 & 0.43 & 0.90 & 0.79 & [143] \\
\hline Benzoylmandeic acid, 4- & A & 23 & 29 & 177 & 0.74 & 1.28 & 1.95 & 1.71 & 1.90 & [77] \\
\hline Benzyl_benzoate & $\mathrm{N}$ & 1 & 11 & 19 & 0.00 & 0.54 & 1.50 & 1.22 & 1.68 & [144] \\
\hline BerberineChloride & B & 56 & 27 & $\underline{175}$ & 0.00 & 1.09 & 2.25 & 2.52 & 2.40 & [145] \\
\hline Betamethasone & $\mathrm{N}$ & -9 & 13 & 232 & 0.80 & $|1.97|$ & 2.95 & 2.07 & 2.91 & {$[99,146-148]$} \\
\hline Betamethasone-17-valerate (excluded ${ }^{* *}$ ) & $\mathrm{N}$ & -29 & 5 & 184 & 0.56 & 2.04 & 3.20 & 1.97 & 3.63 & {$[99,147]$} \\
\hline Bromhexine & B & 50 & 19 & $\underline{137}$ & 0.28 & 0.87 & 1.65 & 1.88 & 2.29 & [149] \\
\hline Budesonide & A & 16 & 26 & $\underline{227}$ & 0.27 & 1.54 & 1.04 & 1.43 & 0.88 & {$[66]$} \\
\hline Bupivacaine & B & 0 & 7 & 107 & 0.26 & 1.19 & 1.59 & 1.32 & 2.51 & {$[8,140,150,151$} \\
\hline Butabarbital & A & 24 & 24 & 127 & 0.52 & 1.21 & 1.36 & 0.98 & 1.66 & [103] \\
\hline Butamben & B & 27 & 12 & 56 & 0.23 & 0.77 & 1.44 & 0.94 & 1.60 & {$[31,57,70,152]$} \\
\hline Butamben & B & 51 & 12 & 58 & 0.23 & 0.77 & 1.44 & 0.94 & 1.60 & [56] \\
\hline Caffeic acid & A & 42 & 30 & 196 & 1.35 & 0.93 & 1.57 & 1.27 & 1.29 & [153] \\
\hline Caffeine & $\mathrm{N}$ & 36 & 20 & 238 & 0.00 & 1.27 & 1.90 & 1.48 & 1.36 & [154] \\
\hline Caffeine & $\mathrm{N}$ & 39 & 20 & 238 & 0.00 & 1.27 & 1.90 & 1.48 & 1.36 & [155] \\
\hline Camptothecin, 10-HO-(lactone) & $A B$ & 52 & 19 & 268 & 0.00 & 0.00 & 0.00 & 0.00 & 0.00 & [156] \\
\hline Camptothecin, 9- $\mathrm{NH}_{2}$-(lactone) & B & 36 & 33 & $\underline{212}$ & 0.40 & 2.27 & 3.74 & 3.12 & 2.53 & [157] \\
\hline Carbamazepine & $\mathrm{N}$ & 24 & 26 & 192 & 0.39 & 0.92 & 2.06 & 2.12 & 1.81 & {$[2,68,158-164]$} \\
\hline Carbenoxolone & A & 20 & 28 & 293 & 1.14 & 1.69 & 2.10 & 1.70 & 4.55 & [165] \\
\hline Carboline, $\beta$ - & B & 17 & 32 & 199 & 0.31 & 0.67 & 1.56 & 1.98 & 1.27 & [166] \\
\hline Carvedilol & B & -12 & 20 & 115 & 0.62 & 2.09 & 3.00 & 3.08 & 3.10 & {$[150,167-169]$} \\
\hline Catechol & A & 27 & 27 & 105 & 0.77 & 0.53 & 1.08 & 0.93 & 0.83 & [63] \\
\hline Catechol, 3- $\mathrm{NO}_{2}^{-}$ & A & 37 & 27 & 86 & 0.58 & 0.50 & 1.41 & 1.10 & 1.01 & [170] \\
\hline Catechol, 4- $\mathrm{NO}_{2}^{-}$ & A & 10 & 31 & 176 & 1.14 & 0.63 & 1.65 & 1.20 & 1.01 & [170] \\
\hline Cefadroxil x $\mathrm{H}_{2} \mathrm{O}$ & $A B$ & 8 & 19 & 197 & 1.55 & 2.82 & 3.48 & 2.76 & 2.49 & [107-172] \\
\hline Cefatrizine.propyleneglycolate (excluded ${ }^{* *}$ ) & $A B$ & 18 & 26 & 204 & 2.19 & 3.41 & 4.46 & 3.92 & 3.04 & {$[112,173]$} \\
\hline Cefpirone Sulfate & $A B$ & 30 & 25 & $\underline{203}$ & 0.50 & 2.77 & 3.99 & 3.71 & 3.46 & [174] \\
\hline Ceftazidime & $A B$ & 23 & 18 & 136 & 1.07 & 3.16 & 4.40 & 3.59 & 3.64 & [175] \\
\hline Celecoxib (excluded) & $A B$ & -66 & 24 & 158 & 0.44 & 1.22 & 2.43 & 2.51 & 2.47 & {$[8,176]$} \\
\hline
\end{tabular}




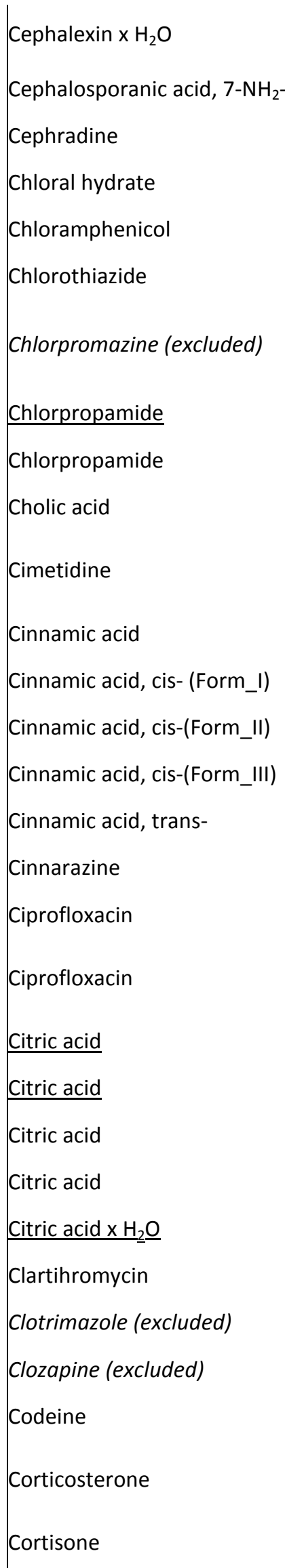

\begin{tabular}{|c|c|c|c|c|c|c|c|c|c|}
\hline$A B$ & 11 & 24 & 327 & 1.06 & 2.54 & 3.27 & 2.53 & 2.43 & {$[2,107,113,177]$} \\
\hline$A B$ & 12 & 17 & 300 & 0.80 & 2.20 & 2.35 & 1.70 & 1.79 & [178] \\
\hline$A B$ & 10 & 14 & 140 & 1.06 & 2.59 & 3.06 & 2.45 & 2.48 & {$[32,68,113]$} \\
\hline $\mathrm{N}$ & 30 & 12 & 57 & 0.00 & 0.30 & 0.99 & 0.54 & 0.77 & [179] \\
\hline $\mathrm{N}$ & 16 & 16 & 151 & 0.87 & 1.65 & 2.66 & 1.84 & 2.07 & {$[8,68,161,180]$} \\
\hline A & 44 & 34 & 350 & 0.64 & 1.66 & 2.74 & 1.98 & 1.69 & [181] \\
\hline B & 80 & 18 & 57 & 0.00 & 0.99 & 1.83 & 2.26 & 2.41 & $\begin{array}{c}{[2,8,25,86,139,16} \\
1,182,184-187]\end{array}$ \\
\hline A & 44 & 29 & 128 & 0.59 & 1.13 & 2.38 & 1.46 & 1.90 & [188] \\
\hline A & 16 & 29 & 128 & 0.59 & 1.13 & 2.38 & 1.46 & 1.90 & {$[8,73,186,189]$} \\
\hline A & 24 & 28 & 200 & 1.51 & 1.66 & 2.66 & 1.82 & 3.31 & [190] \\
\hline B & -13 & 12 & 142 & 0.74 & 1.86 & 1.87 & 1.66 & 1.96 & $\begin{array}{c}{[68,70,79,137,} \\
191]\end{array}$ \\
\hline A & 31 & 27 & 133 & 0.57 & 0.51 & 1.18 & 0.90 & 1.17 & [192] \\
\hline A & 22 & 25 & 42 & 0.57 & 0.51 & 1.18 & 0.90 & 1.17 & [193] \\
\hline A & 22 & 25 & 58 & 0.57 & 0.51 & 1.18 & 0.90 & 1.17 & [193] \\
\hline A & 21 & 25 & 68 & 0.57 & 0.51 & 1.18 & 0.90 & 1.17 & [193] \\
\hline A & 27 & 27 & 133 & 0.57 & 0.51 & 1.18 & 0.90 & 1.17 & [193] \\
\hline B & 48 & 14 & $\underline{112}$ & 0.00 & 1.37 & 2.12 & 2.43 & 3.11 & {$[2,162,194]$} \\
\hline$A B$ & 41 & 21 & 268 & 0.73 & 1.85 & 2.50 & 2.20 & 2.31 & [195] \\
\hline$A B$ & 51 & 21 & 266 & 0.73 & 1.85 & 2.50 & 2.20 & 2.31 & $\begin{array}{c}{[2,79,86,104,} \\
196]\end{array}$ \\
\hline A & 18 & 24 & 153 & 1.63 & 1.33 & 1.50 & 0.61 & 1.24 & [91] \\
\hline A & 23 & 24 & 153 & 1.63 & 1.33 & 1.50 & 0.61 & 1.24 & [197] \\
\hline A & 18 & 24 & 153 & 1.63 & 1.33 & 1.50 & 0.61 & 1.24 & [92] \\
\hline A & 27 & 24 & 153 & 1.63 & 1.33 & 1.50 & 0.61 & 1.24 & [43] \\
\hline A & 29 & 25 & 174 & 1.63 & 1.33 & 1.50 & 0.61 & 1.24 & [91] \\
\hline B & -17 & -31 & 218 & 0.80 & 4.49 & 2.97 & 2.32 & 5.91 & [198] \\
\hline B & -32 & 28 & 148 & 0.00 & 0.78 & 2.37 & 2.48 & 2.62 & [199-201] \\
\hline B & 27 & 18 & 184 & 0.20 & 1.65 & 1.66 & 2.46 & 2.43 & {$[148,169,189]$} \\
\hline B & 8 & 18 & 155 & 0.23 & 1.58 & 1.92 & 2.16 & 2.21 & [202] \\
\hline $\mathrm{N}$ & 10 & 13 & 182 & 0.48 & 1.62 & 2.80 & 1.90 & 2.74 & $\begin{array}{c}{[8,68,147,203-} \\
205]\end{array}$ \\
\hline $\mathrm{N}$ & -8 & 16 & 222 & 0.41 & 1.90 & 3.15 & 2.04 & 2.76 & {$[8,68,147,204]$} \\
\hline
\end{tabular}




\begin{tabular}{|c|c|c|c|c|c|c|c|c|c|c|}
\hline Coumarin & $\mathrm{N}$ & 33 & 14 & 70 & 0.00 & 0.49 & 1.16 & 1.03 & 1.06 & [206] \\
\hline Crotonic acid, trans- (excluded) & A & 79 & 23 & 72 & 0.57 & 0.43 & 0.72 & 0.33 & 0.70 & [207] \\
\hline Cyclacillin (anhyd.) & $A B$ & -18 & 9 & 183 & 1.06 & 2.53 & 2.61 & 2.08 & 2.47 & [208] \\
\hline Cyclacillin (excluded ${ }^{* *}$ ) & $A B$ & 0 & 9 & 183 & 1.06 & 2.53 & 2.61 & 2.08 & 2.47 & {$[111,114]$} \\
\hline Cyclacillin x $2 \mathrm{H}_{2} \mathrm{O}$ & $A B$ & 0 & 10 & 203 & 1.06 & 2.53 & 2.61 & 2.08 & 2.47 & [208] \\
\hline Cyclobarbital & A & 16 & 26 & 173 & 0.52 & 1.28 & 1.50 & 1.32 & 1.79 & [207] \\
\hline Cyclobarbital & A & 27 & 27 & 193 & 0.52 & 1.28 & 1.50 & 1.32 & 1.79 & {$[34,110,209]$} \\
\hline Cyclohexanol & $\mathrm{N}$ & -9 & 6 & 25 & 0.31 & 0.32 & 0.53 & 0.42 & 0.90 & [210] \\
\hline Cyclosporine A & $\mathrm{N}$ & -45 & -40 & 151 & 1.25 & 7.61 & \#\#\#\# & 4.23 & 10.02 & {$[67,211]$} \\
\hline Cyproheptadine (excluded) & B & 30 & 17 & 113 & 0.00 & 0.83 & 1.45 & 2.05 & 2.39 & {$[2,149,150]$} \\
\hline Cystine, L- & $A B$ & 23 & 13 & 260 & 1.56 & 2.09 & 1.98 & 1.44 & 1.63 & [51] \\
\hline Daidzein & A & 66 & 35 & 330 & 1.16 & 1.27 & 2.23 & 2.21 & 1.79 & [212] \\
\hline Danazol & $\mathrm{N}$ & -25 & 21 & 226 & 0.40 & 1.03 & 2.38 & 2.14 & 2.67 & $\begin{array}{c}{[67,99,146,162} \\
213,214]\end{array}$ \\
\hline Dapsone & B & 35 & 28 & 176 & 0.45 & 1.35 & 2.84 & 1.87 & 1.81 & {$[32,68]$} \\
\hline Deferiprone & A & 24 & 20 & $\underline{124}$ & 0.00 & 0.00 & 0.00 & 0.00 & 0.00 & [215] \\
\hline Dehydroisoandrosterone, 5, 6- & $\mathrm{N}$ & 24 & 14 & 141 & 0.31 & 1.01 & 2.27 & 1.55 & 2.38 & [116] \\
\hline Delphinidin_chloride & $A B$ & 20 & 33 & $\underline{224}$ & 2.66 & 1.57 & 2.53 & 3.02 & 2.19 & [216] \\
\hline Deoxyadenosine, 2'- & B & 49 & 27 & 189 & 0.72 & 1.94 & 2.38 & 2.49 & 1.70 & [217] \\
\hline Deoxycorticosterone & $\mathrm{N}$ & -28 & 11 & 142 & 0.17 & 1.32 & 2.55 & 1.69 & 2.68 & {$[32,68,147]$} \\
\hline Deoxyguanosine & $A B$ & 45 & 19 & $\underline{220}$ & 1.09 & 2.58 & 2.56 & 2.36 & 1.75 & [217] \\
\hline Dexamethasone & $\mathrm{N}$ & -11 & 14 & 262 & 0.80 & 1.97 & 2.95 & 2.07 & 2.91 & $\begin{array}{c}{[32,68,78,99,146,} \\
147,159]\end{array}$ \\
\hline Dexamethasone & $\mathrm{N}$ & 15 & 14 & 263 & 0.80 & 1.97 & 2.95 & 2.07 & 2.91 & [218] \\
\hline Dexamethasone & $\mathrm{N}$ & 29 & 14 & 263 & 0.80 & 1.97 & 2.95 & 2.07 & 2.91 & [219] \\
\hline Diatrizoic acid & A & 11 & 37 & 300 & 1.28 & 1.18 & 1.97 & 3.36 & 2.50 & [220] \\
\hline Diazepam & B & 12 & 21 & 132 & 0.00 & 1.04 & 1.72 & 2.11 & 2.07 & $\begin{array}{c}{[8,67,78,140,161,} \\
162,189,221]\end{array}$ \\
\hline Diazinon & B & -17 & 4 & 120 & 0.00 & 1.38 & 1.10 & 1.31 & 2.31 & [222] \\
\hline Diclofenac & $A$ & 20 & 32 & 168 & 0.70 & 0.67 & 1.95 & 1.81 & 2.03 & $\begin{array}{c}{[2,82,102,148} \\
161,223-234]\end{array}$ \\
\hline Diflorason diacetate & $\mathrm{N}$ & 22 & 5 & $\underline{145}$ & 0.38 & 2.08 & 3.52 & 1.82 & 3.53 & {$[235]$} \\
\hline Difloxacin & $A B$ & 0 & 21 & 211 & 0.57 & 1.91 & 2.91 & 2.55 & 2.76 & [104] \\
\hline Diflunisal & A & 22 & 32 & 214 & 0.70 & 0.44 & 1.50 & 1.55 & 1.63 & [236] \\
\hline
\end{tabular}




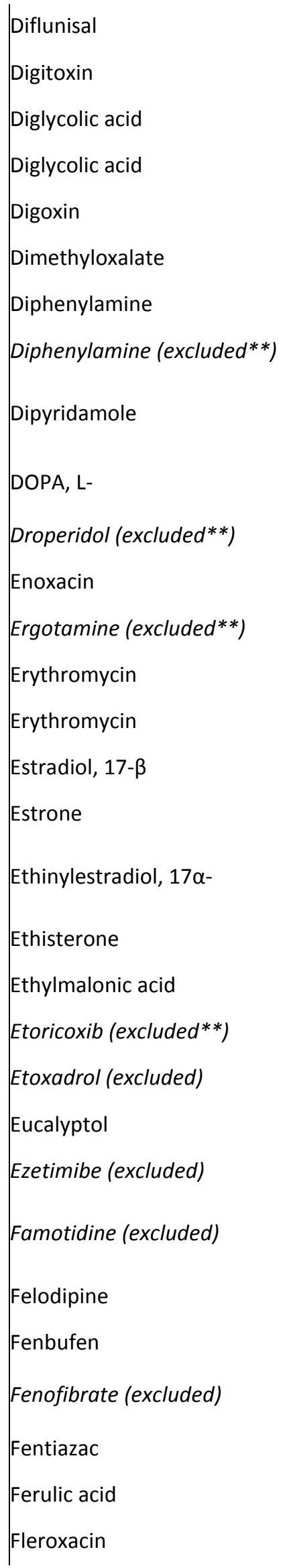

\begin{tabular}{|c|c|c|c|c|c|c|c|c|c|}
\hline A & 37 & 32 & 214 & 0.70 & 0.44 & 1.50 & 1.55 & 1.63 & {$[68,237,238]$} \\
\hline $\mathrm{N}$ & -24 & -9 & 256 & 1.27 & 4.02 & 4.20 & 3.46 & 5.69 & {$[161,239,240]$} \\
\hline A & 23 & 25 & 143 & 1.14 & 0.90 & 1.22 & 0.38 & 0.88 & [43] \\
\hline A & 31 & 25 & 143 & 1.14 & 0.90 & 1.22 & 0.38 & 0.88 & [126] \\
\hline $\mathrm{N}$ & -34 & -9 & 249 & 1.58 & 4.32 & 4.46 & 3.67 & 5.75 & {$[8,78]$} \\
\hline $\mathrm{N}$ & 33 & 4 & 54 & 0.00 & 0.69 & 0.97 & 0.13 & 0.82 & [241] \\
\hline $\mathrm{N}$ & 1 & 16 & 54 & 0.13 & 0.42 & 1.41 & 1.37 & 1.42 & [242] \\
\hline $\mathrm{N}$ & 0 & 16 & 53 & 0.13 & 0.42 & 1.41 & 1.37 & 1.42 & {$[32,243]$} \\
\hline B & 10 & 12 & 165 & 0.95 & 3.03 & 2.90 & 3.74 & 3.87 & $\begin{array}{c}{[99,150,169,199} \\
244-246]\end{array}$ \\
\hline$A B$ & 8 & 20 & 277 & 1.56 & 1.44 & 1.77 & 1.33 & 1.43 & {$[8,110,247,248]$} \\
\hline B & -44 & 22 & 146 & 0.50 & $\mid 1.79$ & 2.77 & 2.78 & 2.82 & {$[169,249]$} \\
\hline$A B$ & 24 & 17 & 222 & 0.73 & $\mid 1.96$ & 2.45 & 2.06 & 2.23 & [104] \\
\hline B & 18 & 24 & 213 & 0.79 & 3.69 & 4.60 & 4.56 & 4.21 & {$[140,188,250]$} \\
\hline B & -23 & -31 & 191 & 1.05 & 4.63 & 3.04 & 2.51 & 5.77 & {$[86,198,251]$} \\
\hline B & -18 & -31 & 191 & 1.05 & 4.63 & 3.04 & 2.51 & 5.77 & [198] \\
\hline A & 52 & 32 & 176 & 0.81 & 0.95 & 2.30 & 1.85 & 2.20 & [252] \\
\hline A & 37 & 34 & 255 & 0.50 & 0.95 & 2.53 & 1.85 & 2.16 & {$[8,68,161,253]$} \\
\hline A & 37 & 32 & 144 & 0.90 & 1.02 & 2.43 & 2.07 & 2.40 & $\begin{array}{c}{[2,32,68,253} \\
254]\end{array}$ \\
\hline $\mathrm{N}$ & 28 & 19 & 269 & 0.40 & 1.08 & 2.40 & 1.78 & 2.58 & [252] \\
\hline A & 8 & 25 & 114 & 1.14 & 0.72 & 1.05 & 0.35 & 0.96 & [255] \\
\hline B & 13 & 26 & 128 & 0.00 & 1.41 & 2.77 & 2.60 & 2.54 & {$[256,257]$} \\
\hline B & 113 & 8 & 124 & 0.15 & 1.05 & 1.24 & 1.20 & 2.13 & [258] \\
\hline $\mathrm{N}$ & -23 & 1 & 37 & 0.00 & 0.32 & 0.39 & 0.43 & 1.36 & 〔259] \\
\hline A & -97 & 30 & 165 & 0.81 & $\mid 1.77$ & 2.61 & 2.65 & 2.94 & {$[260,261]$} \\
\hline B & -60 & 14 & 164 & 1.21 & 2.78 & 2.24 & 2.69 & 2.26 & $\begin{array}{c}{[223,248,262} \\
263]\end{array}$ \\
\hline B & 23 & 8 & 145 & 0.13 & 1.42 & 1.85 & 1.56 & 2.71 & {$[99,264,265]$} \\
\hline A & 41 & 31 & 186 & 0.57 & 0.85 & 2.05 & 1.79 & 1.98 & [266] \\
\hline $\mathrm{N}$ & -49 & 7 & 81 & 0.00 & 1.13 & 2.11 & 1.62 & 2.72 & {$[60,160,267,268]$} \\
\hline$A B$ & 9 & 30 & 161 & 0.57 & 0.81 & 2.29 & 2.62 & 2.29 & [102] \\
\hline A & 36 & 28 & 169 & 0.85 & 0.87 & 1.46 & 1.11 & 1.43 & [153] \\
\hline$A B$ & 12 & 12 & $\underline{184}$ & 0.57 & 1.81 & 2.37 & 1.81 & 2.45 & [104] \\
\hline
\end{tabular}




\begin{tabular}{|c|c|c|c|c|c|c|c|c|c|c|}
\hline Fluconazole & B & 27 & 24 & 139 & 0.31 & 1.42 & 2.45 & 2.16 & 2.01 & [269-272] \\
\hline Flufenamic acid & A & 29 & 28 & 125 & 0.72 & 0.59 & 1.36 & 1.26 & 1.83 & $\begin{array}{c}{[8,68,139,186} \\
226,227,273-275]\end{array}$ \\
\hline Fluorene (excluded ${ }^{* *}$ ) & $\mathrm{N}$ & 4 & 22 & 117 & 0.00 & 0.19 & 1.13 & 1.65 & 1.36 & {$[31,161]$} \\
\hline Fluotrimazole (excluded ${ }^{* *}$ ) & B & 1 & 23 & 132 & 0.00 & 0.66 & 2.14 & 2.15 & 2.65 & {$[32,34]$} \\
\hline Fluphenazine & B & 16 & 11 & $\underline{142}$ & 0.23 & 1.80 & 2.00 & 2.40 & 3.09 & {$[8,25]$} \\
\hline Flurbiprofen & A & 5 & 28 & 111 & 0.57 & 0.58 & 1.51 & 1.50 & 1.84 & $\begin{array}{l}{[2,60,68,102,110} \\
139,148,161,223 \\
236,273,276-278]\end{array}$ \\
\hline Flurbiprofen & A & 43 & 28 & 111 & 0.57 & 0.58 & 1.51 & 1.50 & 1.84 & [236] \\
\hline Folic acid (excluded) & $A B$ & 76 & 19 & 250 & 1.95 & 3.14 & 3.74 & 3.24 & 3.04 & [279] \\
\hline Fumaric acid & A & 32 & 30 & 287 & 1.14 & 0.75 & 1.16 & 0.50 & 0.78 & [63] \\
\hline Furo[3,4-b]quinolin-3(1H)-one, 9- $\mathrm{HO}-1,7-\mathrm{Me}_{2}$ & $A B$ & 24 & 23 & $\underline{200}$ & 0.13 & 1.07 & 1.65 & 1.90 & 1.63 & [38] \\
\hline Furosemide & A & 23 & 31 & 238 & 1.25 & 1.50 & 2.37 & 2.07 & 2.10 & [66] \\
\hline Furosemide & A & 37 & 31 & 238 & 1.25 & 1.50 & 2.37 & 2.07 & 2.10 & $\begin{array}{c}{[2,8,73,148,185,} \\
223,280-284]\end{array}$ \\
\hline Gallic acid & A & 43 & 32 & 251 & 1.66 & 1.01 & 1.64 & 1.29 & 1.11 & [286] \\
\hline Gallic acid & A & 45 & 32 & 251 & 1.66 & 1.01 & 1.64 & 1.29 & 1.11 & [287] \\
\hline Genistein & A & 35 & 35 & 302 & 1.30 & 1.20 & 2.25 & 2.36 & 1.85 & [212] \\
\hline Glibenclamide (excluded) & A & -40 & 32 & 170 & 0.85 & 2.01 & 3.84 & 2.64 & 3.56 & $\begin{array}{l}{[99,158,161,162} \\
189,199,288-290]\end{array}$ \\
\hline Gliclazide & $A B$ & 4 & 17 & 181 & 0.59 & 1.66 & 2.54 & 1.93 & 2.36 & {$[189,289,291]$} \\
\hline Glimepiride (excluded ${ }^{* *}$ ) & A & 16 & 31 & 207 & 0.75 & 2.15 & 3.50 & 2.41 & 3.72 & {$[260,292]$} \\
\hline Glipizide & $A B$ & -14 & 18 & 209 & 0.85 & 2.19 & 3.71 & 2.52 & 3.30 & $\begin{array}{c}{[160,162,227} \\
268]\end{array}$ \\
\hline Glucoside, $\alpha$-D-Me- & $\mathrm{N}$ & 4 & 8 & 168 & 1.00 & 1.83 & 1.50 & 1.19 & 1.34 & [293] \\
\hline Glutamic acid, D- & $A B$ & 26 & 11 & 199 & 1.35 & 1.26 & 1.37 & 0.55 & 1.06 & [51] \\
\hline Glutamic acid, DL- & $A B$ & 26 & 11 & 201 & 1.35 & 1.26 & 1.37 & 0.55 & 1.06 & [51] \\
\hline Glutamic acid, DL- & $A B$ & 27 & 11 & 201 & 1.35 & 1.26 & 1.37 & 0.55 & 1.06 & [45] \\
\hline Glutamic acid, L- & $A B$ & 27 & 11 & 201 & 1.35 & $\mid 1.26$ & 1.37 & 0.55 & 1.06 & [51] \\
\hline Glutamic acid, L- & $A B$ & 30 & 11 & 201 & 1.35 & 1.26 & 1.37 & 0.55 & 1.06 & [54] \\
\hline Glutaric acid & A & 25 & 25 & 97 & 1.14 & 0.69 & 1.07 & 0.34 & 0.96 & [29] \\
\hline Glutaric acid & A & 26 & 25 & 97 & 1.14 & 0.69 & 1.07 & 0.34 & 0.96 & [43] \\
\hline Glutaric acid & A & 31 & 25 & 97 & 1.14 & 0.69 & 1.07 & 0.34 & 0.96 & [44] \\
\hline
\end{tabular}




\begin{tabular}{|c|c|c|c|c|c|c|c|c|c|c|}
\hline Glutethimide & A & 41 & 25 & 84 & 0.34 & 1.02 & 1.40 & 1.31 & 1.73 & [294] \\
\hline Glutethimide & A & 21 & 25 & 84 & 0.34 & 1.02 & 1.40 & 1.31 & 1.73 & [295] \\
\hline Glutethimide $\mathrm{x}_{2} \underline{O}$ & A & 49 & 25 & 84 & 0.34 & 1.02 & 1.40 & 1.31 & 1.73 & [294] \\
\hline Glycine & $A B$ & 14 & 16 & 240 & 0.78 & 0.90 & 0.93 & 0.37 & 0.57 & [51] \\
\hline Glycine & $A B$ & 12 & 16 & 245 & 0.78 & 0.90 & 0.93 & 0.37 & 0.57 & [63] \\
\hline Glycine & $A B$ & 18 & 17 & 262 & 0.78 & 0.90 & 0.93 & 0.37 & 0.57 & {$[94,97,296-299]$} \\
\hline Glycolic acid & A & 2 & 23 & 77 & 0.74 & 0.63 & 0.67 & 0.30 & 0.52 & [126] \\
\hline Glycolic acid & A & 11 & 23 & 77 & 0.74 & 0.63 & 0.67 & 0.30 & 0.52 & [126] \\
\hline Griseofulvin & $\mathrm{N}$ & 4 & 13 & 220 & 0.00 & 1.53 & 2.26 & 1.74 & 2.44 & [188] \\
\hline Griseofulvin & $\mathrm{N}$ & 28 & 13 & 220 & 0.00 & 1.53 & 2.26 & 1.74 & 2.44 & $\begin{array}{c}{[32,67,68,79,99} \\
199,300]\end{array}$ \\
\hline Griseofulvin & $\mathrm{N}$ & 35 & 14 & 220 & 0.00 & 1.58 & 2.32 & 1.86 & 2.40 & [295] \\
\hline Haloperidol & B & 42 & 13 & 149 & 0.31 & 1.45 & 2.08 & 2.00 & 2.80 & $\begin{array}{c}{[68,139,150,194} \\
301]\end{array}$ \\
\hline Hemimellitic acid & A & 44 & 30 & 200 & 1.71 & 1.10 & 1.84 & 1.14 & 1.36 & [131] \\
\hline Heptabarbital & A & 23 & 26 & 174 & 0.52 & 1.28 & 1.50 & 1.32 & 1.93 & [103] \\
\hline Hexaglycine (excluded ${ }^{* *}$ ) & $A B$ & 16 & 10 & 141 & 2.06 & 3.71 & 5.32 & 1.91 & 2.55 & {$[247,299]$} \\
\hline Hexobarbital & A & 30 & 26 & 147 & 0.24 & 1.33 & 1.50 & 1.34 & 1.79 & {$[8,186]$} \\
\hline Hexobarbital & A & 35 & 26 & 146 & 0.24 & 1.33 & 1.50 & 1.34 & 1.79 & [130] \\
\hline Hippuric acid & A & 30 & 29 & 187 & 0.83 & 1.01 & 1.95 & 1.06 & 1.33 & [302] \\
\hline Histidine, L- & $A B$ & 13 & 24 & 282 & 0.35 & 0.82 & 1.56 & 1.07 & 1.13 & {$[51]$} \\
\hline Hydrastine (excluded ${ }^{* *}$ ) & B & 2 & 16 & 132 & 0.00 & 1.89 & 2.60 & 2.33 & 2.68 & {$[32,34]$} \\
\hline Hydrobenzoin (excluded ${ }^{* *}$ ) & $\mathrm{N}$ & 0 & 14 & 138 & 0.54 & 1.09 & 1.47 & 1.61 & 1.72 & {$[32,70]$} \\
\hline Hydrochlorothiazide & A & 12 & 32 & 274 & 1.01 & 1.76 & 2.77 & 2.15 & 1.73 & {$\left[\begin{array}{c}{[32,68,79,160,162} \\
163,223,280]\end{array}\right.$} \\
\hline Hydrochlorothiazide & A & 33 & 32 & 274 & 1.01 & 1.76 & 2.77 & 2.15 & 1.73 & [181] \\
\hline Hydrochlorothiazide & A & 40 & 32 & 274 & 1.01 & 1.76 & 2.77 & 2.15 & 1.73 & [303] \\
\hline Hydrocortisone & $\mathrm{N}$ & -6 & 14 & 219 & 0.73 & 1.90 & 2.92 & 2.04 & 2.80 & $\begin{array}{c}{[32,68,146,147} \\
148,159,163]\end{array}$ \\
\hline Hydrocortisone & $\mathrm{N}$ & 20 & 14 & 219 & 0.73 & 1.90 & 2.92 & 2.04 & 2.80 & {$[219]$} \\
\hline Hydroquinone & A & 20 & 29 & 174 & 1.00 & 0.65 & 1.14 & 1.02 & 0.83 & [63] \\
\hline Hydroxyprogesterone, $17-\alpha$ & $\mathrm{N}$ & 30 & 14 & 222 & 0.17 & 1.32 & 2.50 & 1.66 & 2.68 & [252] \\
\hline Hydroxyproline, L- & $A B$ & 6 & 16 & 272 & 0.95 & 1.20 & 1.08 & 0.77 & 0.94 & [51] \\
\hline
\end{tabular}




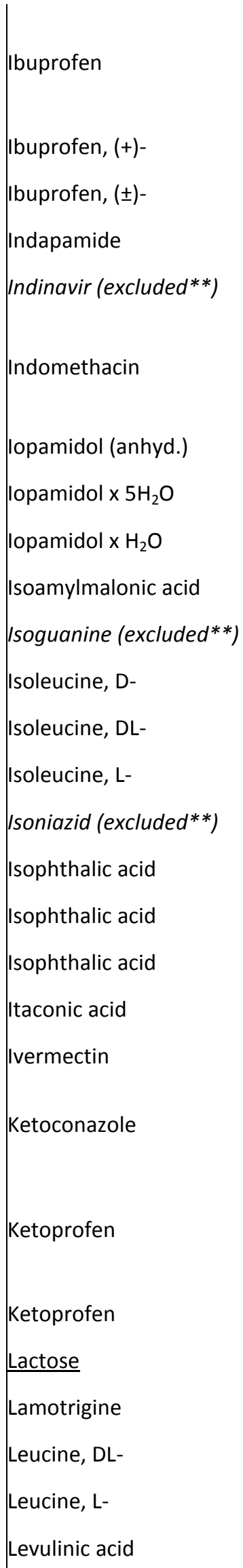

\begin{tabular}{|c|c|c|c|c|c|c|c|c|c|}
\hline A & 14 & 24 & 76 & 0.57 & 0.51 & 1.01 & 0.78 & 1.78 & $\begin{array}{c}189,223,225,273, \\
304-307]\end{array}$ \\
\hline$A$ & 24 & 24 & 76 & 0.57 & 0.51 & 1.01 & 0.78 & 1.78 & [236] \\
\hline$A$ & 29 & 24 & 76 & 0.57 & 0.51 & 1.01 & 0.78 & 1.78 & {$[236]$} \\
\hline$A$ & 14 & 32 & 161 & 0.70 & 1.86 & 3.20 & 2.64 & 2.50 & {$[68,239,308]$} \\
\hline B & -23 & 6 & 168 & 0.98 & 3.59 & 4.27 & 3.63 & 4.90 & {$[309,310]$} \\
\hline$A$ & 23 & 32 & 159 & 0.57 & 1.24 & 2.49 & 2.44 & 2.53 & $\begin{array}{c}{[86,138,161,162,} \\
164,225,273,311- \\
316]\end{array}$ \\
\hline $\mathrm{N}$ & -5 & 24 & $\underline{189}$ & 2.20 & 3.48 & 4.72 & 4.41 & 3.68 & [317] \\
\hline $\mathrm{N}$ & 56 & 24 & $\underline{189}$ & 2.20 & 3.48 & 4.72 & 4.41 & 3.68 & [317] \\
\hline $\mathrm{N}$ & 9 & 24 & $\underline{189}$ & 2.20 & 3.48 & 4.72 & 4.41 & 3.68 & [317] \\
\hline A & 11 & 25 & $\underline{111}$ & 1.14 & 0.76 & 1.05 & 0.35 & 1.39 & [255] \\
\hline$A B$ & -6 & 24 & 360 & 0.60 & 1.80 & 1.40 & 1.62 & 0.98 & {$[33,247]$} \\
\hline$A B$ & 3 & 11 & 265 & 0.78 & 0.97 & 0.92 & 0.39 & 1.13 & [51] \\
\hline$A B$ & 8 & 12 & 280 & 0.78 & 0.97 & 0.92 & 0.39 & 1.13 & [51] \\
\hline$A B$ & 4 & 12 & 280 & 0.78 & 0.97 & 0.92 & 0.39 & 1.13 & [51] \\
\hline B & 0 & 21 & 171 & 0.47 & 1.39 & 1.85 & 1.19 & 1.03 & {$[248,318]$} \\
\hline$A$ & 34 & 33 & 347 & 1.14 & 0.77 & 1.46 & 0.94 & 1.15 & [43] \\
\hline$A$ & 48 & 33 & 345 & 1.14 & 0.77 & 1.46 & 0.94 & 1.15 & [319] \\
\hline$A$ & 66 & 33 & 347 & 1.14 & 0.77 & 1.46 & 0.94 & 1.15 & [131] \\
\hline$A$ & 34 & 27 & 175 & 1.14 & 0.76 & 1.07 & 0.44 & 0.92 & [45] \\
\hline $\mathrm{N}$ & -25 & -32 & 140 & 0.68 & 4.23 & 3.21 & 3.24 & 6.72 & {$[101,300,320]$} \\
\hline B & 10 & 21 & 146 & 0.00 & 2.22 & 3.76 & 3.14 & 3.72 & $\begin{array}{c}{[146,161,162,169,} \\
205,321-323]\end{array}$ \\
\hline$A$ & 21 & 28 & 94 & 0.57 & 0.87 & 1.97 & 1.56 & 1.98 & $\begin{array}{c}{[2,32,68,79,82,} \\
110,161,189,223, \\
225,273,324]\end{array}$ \\
\hline$A$ & 34 & 28 & 94 & 0.57 & 0.87 & 1.97 & 1.56 & 1.98 & [236] \\
\hline $\mathrm{N}$ & 16 & 4 & 202 & 2.01 & 3.40 & 2.69 & 2.33 & 2.23 & [197] \\
\hline B & 66 & 35 & 217 & 0.45 & 0.93 & 2.13 & 2.40 & 1.65 & [325-328] \\
\hline$A B$ & 9 & 12 & 293 & 0.78 & 0.97 & 0.92 & 0.39 & 1.13 & [51] \\
\hline$A B$ & 3 & 12 & 293 & 0.78 & 0.97 & 0.92 & 0.39 & 1.13 & [51] \\
\hline$A$ & 27 & 22 & 31 & 0.57 & 0.67 & 1.12 & 0.38 & 0.90 & [43] \\
\hline
\end{tabular}




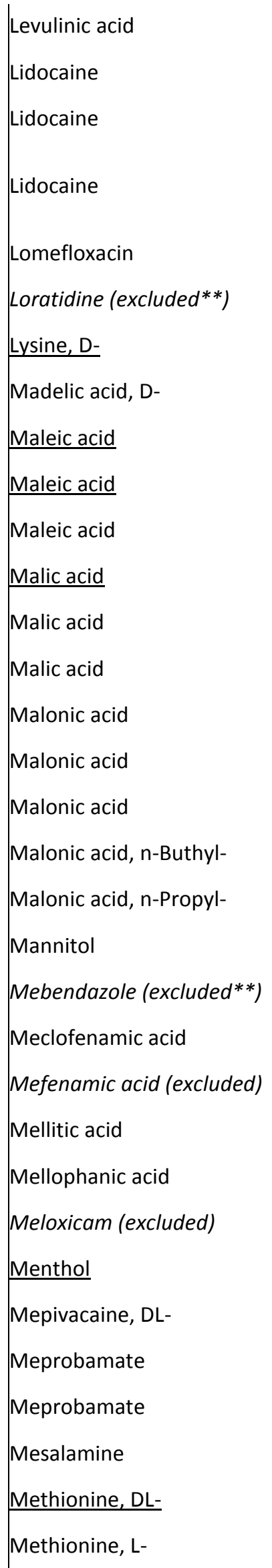

\begin{tabular}{|c|c|c|c|c|c|c|c|c|c|}
\hline A & 43 & 22 & 31 & 0.57 & 0.67 & 1.12 & 0.38 & 0.90 & [126] \\
\hline B & -9 & 6 & 69 & 0.26 & 1.17 & 1.50 & 1.10 & 2.06 & [329] \\
\hline B & -9 & 6 & 69 & 0.26 & 1.17 & $\mid 1.50$ & 1.10 & 2.06 & [330] \\
\hline B & -4 & 6 & 69 & 0.26 & 1.17 & 1.50 & 1.10 & 2.06 & $\begin{array}{c}{[32,82,110,150,} \\
203,305,331]\end{array}$ \\
\hline$A B$ & 10 & 15 & 240 & 0.73 & 1.81 & 2.37 & 1.87 & 2.43 & [104] \\
\hline B & -29 & 17 & 135 & 0.00 & 1.14 & 2.09 & 2.19 & 2.87 & {$[169,322]$} \\
\hline$A B$ & -15 & 8 & 217 & 0.99 & 1.48 & 1.26 & 0.58 & 1.23 & [51] \\
\hline A & 60 & 25 & 132 & 0.74 & 0.89 & 1.05 & 0.90 & 1.13 & [77] \\
\hline A & 19 & 26 & 134 & 1.14 & 0.75 & $\mid 1.16$ & 0.50 & 0.78 & [197] \\
\hline A & 21 & 26 & 134 & 1.14 & 0.75 & 1.16 & 0.50 & 0.78 & [91] \\
\hline A & 24 & 26 & 134 & 1.14 & 0.75 & $\mid 1.16$ & 0.50 & 0.78 & [92] \\
\hline A & 22 & 24 & 131 & 1.14 & 0.99 & 1.10 & 0.47 & 0.88 & [91] \\
\hline A & 12 & 24 & 131 & 1.14 & 0.99 & 1.10 & 0.47 & 0.88 & [43] \\
\hline A & 13 & 24 & 131 & 1.14 & 0.99 & 1.10 & 0.47 & 0.88 & [92] \\
\hline A & 4 & 26 & 135 & 1.14 & 0.69 & $\mid 1.06$ & 0.34 & 0.68 & [333] \\
\hline A & 9 & 26 & 136 & 1.14 & 0.69 & $\mid 1.06$ & 0.34 & 0.68 & [43] \\
\hline A & 12 & 26 & 136 & 1.14 & 0.69 & $\mid 1.06$ & 0.34 & 0.68 & [92] \\
\hline A & 21 & 25 & 102 & 1.14 & 0.73 & $\mid 1.06$ & 0.34 & 1.24 & [255] \\
\hline A & 10 & 25 & $\underline{114}$ & 1.14 & 0.72 & $\mid 1.06$ & 0.35 & 1.10 & [255] \\
\hline $\mathrm{N}$ & 19 & 10 & 167 & 1.62 & 1.81 & 1.75 & 1.23 & 1.31 & [63] \\
\hline$A B$ & 37 & 32 & 289 & 0.71 & 1.38 & 2.76 & 2.45 & 2.13 & {$[334,335]$} \\
\hline A & 13 & 33 & 257 & 0.65 & 0.62 & 1.68 & 1.87 & 2.03 & {$[139,226,246]$} \\
\hline A & 48 & 31 & 231 & 0.65 & 0.70 & 1.47 & 1.65 & 1.92 & [205] \\
\hline A & 4 & 22 & 287 & 3.43 & 2.08 & 2.99 & 1.71 & 2.01 & [131] \\
\hline A & 33 & 28 & $\underline{212}$ & 2.29 & 1.43 & 2.22 & 1.33 & 1.58 & [131] \\
\hline$A B$ & -43 & 27 & 254 & 0.72 & 2.02 & 3.12 & 2.60 & 2.32 & [336] \\
\hline $\mathrm{N}$ & 0 & 1 & 43 & 0.31 & 0.42 & 0.50 & 0.44 & 1.47 & [197] \\
\hline B & -12 & 12 & 150 & 0.26 & 1.18 & 1.58 & 1.32 & 2.09 & [330] \\
\hline $\mathrm{N}$ & 41 & 5 & 104 & 0.89 & 1.12 & $\mid 1.62$ & 0.71 & 1.73 & [337] \\
\hline $\mathrm{N}$ & 42 & 5 & 104 & 0.89 & 1.12 & $\mid 1.62$ & 0.71 & 1.73 & [337] \\
\hline$A B$ & 18 & 27 & 280 & 0.93 & 0.70 & 1.52 & 1.22 & 1.09 & [338] \\
\hline$A B$ & 18 & 15 & 281 & 0.78 & 1.06 & 1.08 & 0.72 & 1.15 & [51] \\
\hline$A B$ & 12 & 15 & 278 & 0.78 & 1.06 & $\mid 1.08$ & 0.72 & 1.15 & [51] \\
\hline
\end{tabular}




\begin{tabular}{|c|c|c|c|c|c|c|c|c|c|c|}
\hline Methylmalonic acid & A & 10 & 26 & 130 & 1.14 & 0.72 & 1.05 & 0.35 & 0.82 & [255] \\
\hline Methylprednisolone (Form II) & $\mathrm{N}$ & 21 & 15 & 233 & 0.73 & 2.00 & 3.00 & 2.20 & 2.90 & [339] \\
\hline Metiazinic acid (excluded) & A & 95 & 31 & 144 & 0.57 & 0.89 & 1.93 & 2.16 & 1.98 & [8] \\
\hline Metolazone & A & 30 & 34 & 256 & 0.59 & 1.74 & 2.86 & 2.69 & 2.50 & [340] \\
\hline Metronidazole & B & 6 & 22 & 159 & 0.31 & 0.86 & 1.75 & 1.12 & 1.19 & $\begin{array}{c}{[32,34,68,183} \\
341]\end{array}$ \\
\hline Morphine & $A B$ & -1 & 21 & 254 & 0.50 & 1.47 & 1.59 & 2.23 & 2.07 & {$[305,342,343]$} \\
\hline m-toluic acid (excluded) & A & 71 & 26 & 110 & 0.57 & 0.44 & 1.02 & 0.77 & 1.07 & [53] \\
\hline $\mathrm{N}$-(2,3-dichlorophenyl)benzenesulfonamide & A & 28 & 31 & 114 & 0.43 & 0.74 & 2.21 & 1.90 & 1.95 & [134] \\
\hline $\mathrm{N}$-(2-chlorophenyl)benzenesulfonamide & A & 13 & 30 & 125 & 0.36 & 0.81 & 2.14 & 1.79 & 1.83 & [134] \\
\hline N-(4-chlorophenyl)benzenesulfonamide & A & 39 & 30 & 122 & 0.42 & 0.81 & 2.17 & 1.82 & 1.83 & [134] \\
\hline N,2-dimethylpropanamide & $\mathrm{N}$ & -16 & 6 & $\underline{62}$ & 0.26 & 0.62 & 1.04 & 0.32 & 0.93 & [41] \\
\hline $\mathrm{N}$-acetyl-L-phenylalanine, Ethyl ester & $\mathrm{N}$ & 20 & 7 & $\underline{85}$ & 0.26 & 1.05 & 1.91 & 0.95 & 1.89 & {$[344]$} \\
\hline Nalidixic acid & $A B$ & -19 & 20 & 230 & 0.57 & 1.34 & 1.94 & 1.63 & 1.70 & $\begin{array}{c}{[68,104,161,248} \\
365]\end{array}$ \\
\hline Naphthoic acid, 2- & A & 54 & 30 & 185 & 0.57 & 0.50 & 1.40 & 1.47 & 1.30 & [345] \\
\hline Naphthol, 1- & A & 25 & 28 & 96 & 0.50 & 0.45 & 1.23 & 1.50 & 1.14 & [346] \\
\hline Naphthol, 2- & A & 26 & 28 & 121 & 0.50 & 0.45 & 1.23 & 1.50 & 1.14 & [347] \\
\hline Naphthylamine-1-sulfonic acid, 2- & $A B$ & 22 & 27 & 180 & 0.54 & 1.25 & 2.49 & 1.87 & 1.53 & [136] \\
\hline Naphthylamine-5-sulfonic acid, 1- & $A B$ & 25 & 32 & 300 & 0.54 & 1.25 & 2.52 & 1.89 & 1.53 & {$[136]$} \\
\hline Naproxen & A & 28 & 29 & 153 & 0.57 & 0.75 & 1.49 & 1.54 & 1.78 & [236] \\
\hline Naringin & A & 26 & 11 & 83 & 2.10 & 3.86 & 4.09 & 3.97 & 3.89 & {$[248,348]$} \\
\hline $\mathrm{N}$-butylacetamide & $\mathrm{N}$ & -15 & 5 & $\underline{55}$ & 0.26 & 0.60 & 1.06 & 0.31 & 1.07 & [41] \\
\hline Nevirapine & B & -6 & 31 & 248 & 0.42 & 1.37 & 2.29 & 2.36 & 1.95 & [349] \\
\hline Nicotinic acid & $A B$ & 14 & 20 & 237 & 0.57 & 0.73 & 1.21 & 0.79 & 0.89 & [43] \\
\hline Nicotinic acid & $A B$ & 20 & 20 & 237 & 0.57 & 0.73 & 1.21 & 0.79 & 0.89 & {$[8,350]$} \\
\hline Nifedipine & B & 42 & 13 & 173 & 0.13 & 1.53 & 2.25 & 1.56 & 2.50 & $\begin{array}{c}{[162,239,265,351,} \\
352]\end{array}$ \\
\hline Niflumic acid & $A B$ & 29 & 18 & 204 & 0.72 & 0.77 & 1.42 & 1.33 & 1.79 & {$\left[\begin{array}{c}{[185,226,273,283} \\
353]\end{array}\right.$} \\
\hline $\mathrm{N}$-isopropylacetamide & $\mathrm{N}$ & -17 & 6 & $\underline{66}$ & 0.26 & 0.62 & 1.04 & 0.32 & 0.93 & {$[41]$} \\
\hline Nitrendipine & B & 45 & 11 & 158 & 0.13 & 1.54 & 2.26 & 1.56 & 2.64 & {$[205,265,300]$} \\
\hline Nitroaminoguanidine (excluded) & B & 112 & 15 & 185 & 0.69 & 1.69 & 1.18 & 1.06 & 0.78 & [354] \\
\hline Nitrofurantoin & A & 32 & 31 & 268 & 0.24 & 1.34 & 2.03 & 1.65 & 1.45 & $\begin{array}{c}{[68,78,139,185,18} \\
6,280,355]\end{array}$ \\
\hline
\end{tabular}




\begin{tabular}{|c|c|c|c|c|c|c|c|c|c|c|}
\hline Nitrofurantoin & A & 44 & 31 & 268 & 0.24 & 1.34 & 2.03 & 1.65 & 1.45 & [356] \\
\hline Nitroglycerin & $\mathrm{N}$ & 7 & 11 & 14 & 0.00 & 0.45 & 1.87 & 0.58 & 1.23 & [357] \\
\hline N-methylacetamide & $\mathrm{N}$ & -13 & 8 & 28 & 0.26 & 0.59 & 1.05 & 0.32 & 0.65 & [62] \\
\hline N-methylacetamide & $\mathrm{N}$ & -4 & 8 & 28 & 0.26 & 0.59 & 1.05 & 0.32 & 0.65 & [41] \\
\hline N-methylpivalamide & $\mathrm{N}$ & -6 & 5 & 91 & 0.26 & 0.63 & 0.99 & 0.29 & 1.07 & [41] \\
\hline N-octylacetamide & $\mathrm{N}$ & -5 & 0 & $\underline{60}$ & 0.26 & 0.61 & 1.08 & 0.30 & 1.63 & [41] \\
\hline Norethindone & $\mathrm{N}$ & 22 & 19 & 203 & 0.40 & 1.07 & 2.44 & 1.81 & 2.44 & [358] \\
\hline Norethindone acetate & $\mathrm{N}$ & 30 & 13 & 161 & 0.09 & 1.13 & 2.58 & 1.66 & 2.74 & [358] \\
\hline Norfloxacin & $A B$ & 19 & 17 & 221 & 0.73 & 1.84 & 2.43 & 1.98 & 2.27 & {$[2,68,104,148]$} \\
\hline Norfloxacin & $A B$ & 26 & 17 & 215 & 0.73 & 1.84 & 2.43 & 1.98 & 2.27 & [195] \\
\hline Norleucine, DL- & $A B$ & 11 & 13 & 299 & 0.78 & 0.94 & 0.94 & 0.38 & 1.13 & [51] \\
\hline Noscapine & B & 44 & 18 & 176 & 0.00 & 2.09 & 3.09 & 2.39 & 2.88 & [8] \\
\hline N-propylacetamide & $\mathrm{N}$ & -16 & 6 & $\underline{57}$ & 0.26 & 0.60 & 1.06 & 0.31 & 0.93 & [41] \\
\hline $\mathrm{N}$-tert-butylacetamide & $\mathrm{N}$ & -3 & 6 & 97 & 0.26 & 0.63 & 0.99 & 0.29 & 1.07 & [41] \\
\hline Ofloxacin & $A B$ & 4 & 18 & 254 & 0.57 & 2.05 & 2.58 & 2.26 & 2.50 & [104] \\
\hline Olmesartan Medoxomil (excluded ${ }^{* *}$ ) & $A B$ & -60 & 19 & 181 & 0.95 & 2.61 & 3.72 & 3.77 & 4.04 & {$[359,360]$} \\
\hline Oxalic acid & A & 9 & 27 & 190 & 1.14 & 0.68 & 1.06 & 0.34 & 0.54 & [91] \\
\hline Oxalic acid & A & 26 & 27 & 190 & 1.14 & 0.68 & 1.06 & 0.34 & 0.54 & [43] \\
\hline Oxalic acid & A & 34 & 27 & 190 & 1.14 & 0.68 & 1.06 & 0.34 & 0.54 & [92] \\
\hline Oxalic acid $\times 2 \mathrm{H}_{2} \underline{\mathrm{O}}$ & A & 35 & 25 & 102 & 1.14 & 0.68 & 1.06 & 0.34 & 0.54 & [91] \\
\hline Oxazepam & B & 6 & 25 & 206 & 0.64 & 1.29 & 1.75 & 2.23 & 1.99 & {$[67,203,361]$} \\
\hline Oxyphenbutazone & A & 52 & 28 & 96 & 0.50 & 1.89 & 2.69 & 2.38 & 2.49 & {$[32,82,273]$} \\
\hline Papaverine & B & -8 & 21 & 147 & 0.00 & 1.47 & 2.76 & 2.19 & 2.59 & {$\left[\begin{array}{l}{[139,169,186,283} \\
362,363,365,366]\end{array}\right.$} \\
\hline Paraben, Buthyl- & A & 30 & 25 & 69 & 0.66 & 0.73 & 1.26 & 0.87 & 1.55 & [65] \\
\hline Paraben, Buthyl- & A & 41 & 25 & 69 & 0.66 & 0.73 & 1.26 & 0.87 & 1.55 & $\begin{array}{c}{[8,31,57,68,331} \\
367]\end{array}$ \\
\hline Paraben, Ethyl- & A & 29 & 27 & 117 & 0.66 & 0.69 & 1.33 & 1.15 & 1.25 & {$[65]$} \\
\hline Paraben, Methyl- & A & 21 & 26 & 131 & 0.66 & 0.72 & 1.24 & 0.87 & 1.13 & $\begin{array}{c}{[31,32,57,68,70} \\
331,367]\end{array}$ \\
\hline Paraben, Methyl- & A & 28 & 27 & 131 & 0.66 & 0.68 & 1.32 & 1.15 & 1.11 & [65] \\
\hline Paraben, Propyl- & A & 34 & 27 & 97 & 0.66 & 0.69 & 1.33 & 1.15 & 1.39 & [65] \\
\hline Paraben, Propyl- & A & 44 & 25 & 97 & 0.66 & 0.73 & 1.25 & 0.87 & 1.41 & $\begin{array}{c}{[8,31,57,68,70,17} \\
1,331,367]\end{array}$ \\
\hline
\end{tabular}




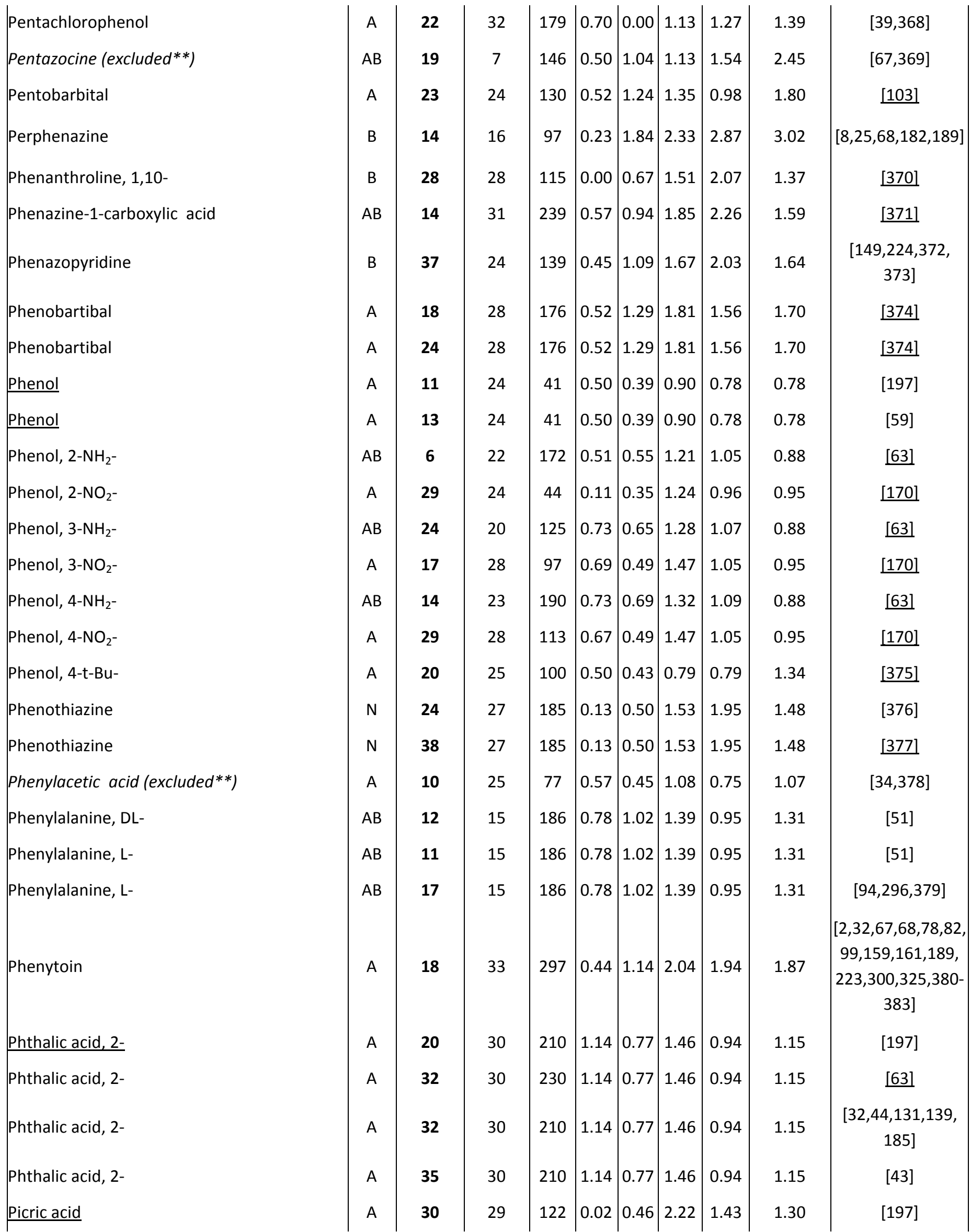




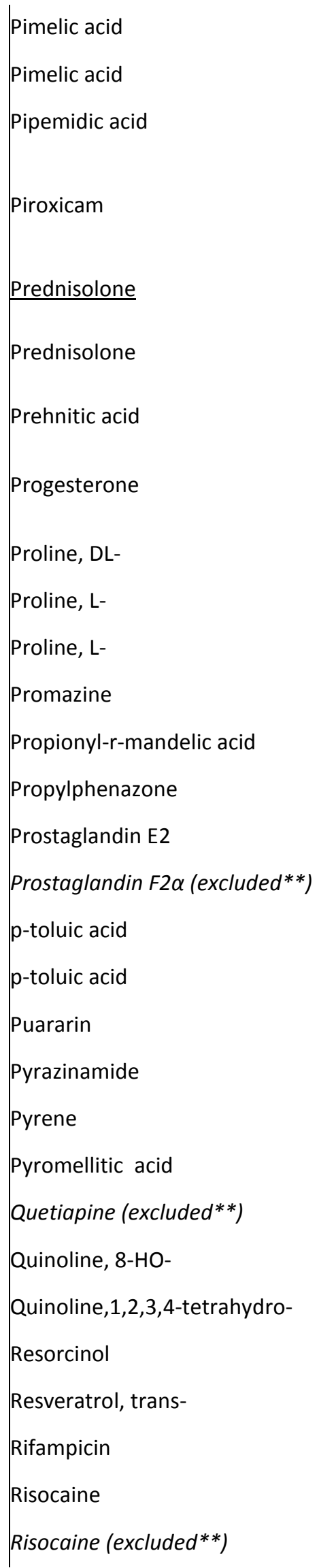

\begin{tabular}{|c|c|c|c|c|c|c|c|c|c|}
\hline A & 36 & 25 & 105 & 1.14 & 0.70 & 1.08 & 0.33 & 1.24 & [44] \\
\hline A & 42 & 25 & 105 & 1.14 & $0.70 \mid$ & 1.08 & 0.33 & 1.24 & [43] \\
\hline$A B$ & 5 & 19 & 254 & 0.73 & 2.07 & 2.48 & 2.20 & 2.17 & [8] \\
\hline$A B$ & 23 & 24 & 199 & 0.44 & 2.17 & 3.09 & 2.66 & 2.25 & $\begin{array}{c}{[2,32,148,161} \\
185,205,273,280 \\
384,385]\end{array}$ \\
\hline $\mathrm{N}$ & 17 & 17 & 239 & 0.73 & $|1.97|$ & 3.02 & 2.19 & 2.76 & [188] \\
\hline $\mathrm{N}$ & 17 & 17 & 239 & 0.73 & $|1.97|$ & 3.02 & 2.19 & 2.76 & $\begin{array}{c}{[31,32,68,147} \\
162,386]\end{array}$ \\
\hline A & 17 & 28 & $\underline{211}$ & 2.29 & 1.43 & 2.22 & 1.33 & 1.58 & [131] \\
\hline $\mathrm{N}$ & 2 & 12 & 126 & 0.00 & 1.04 & 2.49 & 1.56 & 2.62 & $\begin{array}{c}{[8,68,147,148,} \\
159,161,199,204]\end{array}$ \\
\hline$A B$ & 7 & 14 & 208 & 0.71 & 0.81 & 0.87 & 0.54 & 0.88 & [121] \\
\hline$A B$ & 3 & 15 & 228 & 0.71 & 0.81 & 0.87 & 0.54 & 0.88 & [387] \\
\hline$A B$ & 5 & 14 & 221 & 0.71 & 0.81 & 0.87 & 0.54 & 0.88 & [51] \\
\hline B & 23 & 16 & 33 & 0.00 & $\mid 1.06$ & $\mid 1.74$ & 2.13 & 2.28 & {$[8,25,182]$} \\
\hline A & 33 & 26 & 126 & 0.57 & 0.81 & 1.47 & 0.82 & 1.57 & [77] \\
\hline B & 0 & 12 & 103 & 0.00 & 1.33 & $\mid 1.69$ & 1.40 & 1.91 & [119] \\
\hline A & 13 & 23 & 67 & 1.20 & 1.48 & 1.95 & 1.32 & 2.94 & [388] \\
\hline A & 4 & 22 & 30 & 1.51 & $\mid 1.48$ & 1.72 & 1.32 & 2.98 & {$[31,389]$} \\
\hline A & 24 & 28 & 184 & 0.57 & 0.44 & $\mid 1.02$ & 0.77 & 1.07 & [54] \\
\hline A & 43 & 28 & 184 & 0.57 & 0.44 & 1.02 & 0.77 & 1.07 & [53] \\
\hline A & 22 & 22 & 189 & 0.00 & $0.00 \mid$ & 0.00 & 0.00 & 0.00 & [390] \\
\hline B & -11 & 24 & 190 & 0.49 & 1.04 & 1.68 & 1.07 & 0.89 & {$[68,70,391]$} \\
\hline$N$ & 42 & 33 & 152 & 0.00 & 0.25 & 1.52 & 2.60 & 1.59 & [118] \\
\hline A & 50 & 30 & 278 & 2.29 & 1.43 & 2.22 & 1.33 & 1.58 & [131] \\
\hline B & -31 & 14 & 163 & 0.23 & 2.01 & 1.93 & 2.72 & 2.91 & {$[171,363]$} \\
\hline$A B$ & 27 & 20 & 76 & 0.07 & 0.73 & $\mid 1.36$ & 1.55 & 1.10 & [392] \\
\hline B & 11 & 15 & 16 & 0.13 & 0.39 & 0.94 & 1.01 & 1.13 & [393] \\
\hline A & 10 & 27 & 110 & 1.00 & 0.61 & $\mid 1.10$ & 0.99 & 0.83 & [63] \\
\hline A & 30 & 33 & 254 & $\mid 1.50$ & 1.04 & 1.82 & 1.97 & 1.74 & [394] \\
\hline$A B$ & -9 & -8 & $\underline{164}$ & 2.55 & 4.66 & 4.67 & 4.73 & 6.21 & [395-398] \\
\hline B & 31 & 14 & 76 & 0.23 & 0.77 & 1.43 & 0.94 & 1.45 & [55] \\
\hline B & 18 & 14 & 74 & 0.23 & 0.77 & $\mid 1.43$ & 0.94 & 1.45 & [55] \\
\hline
\end{tabular}




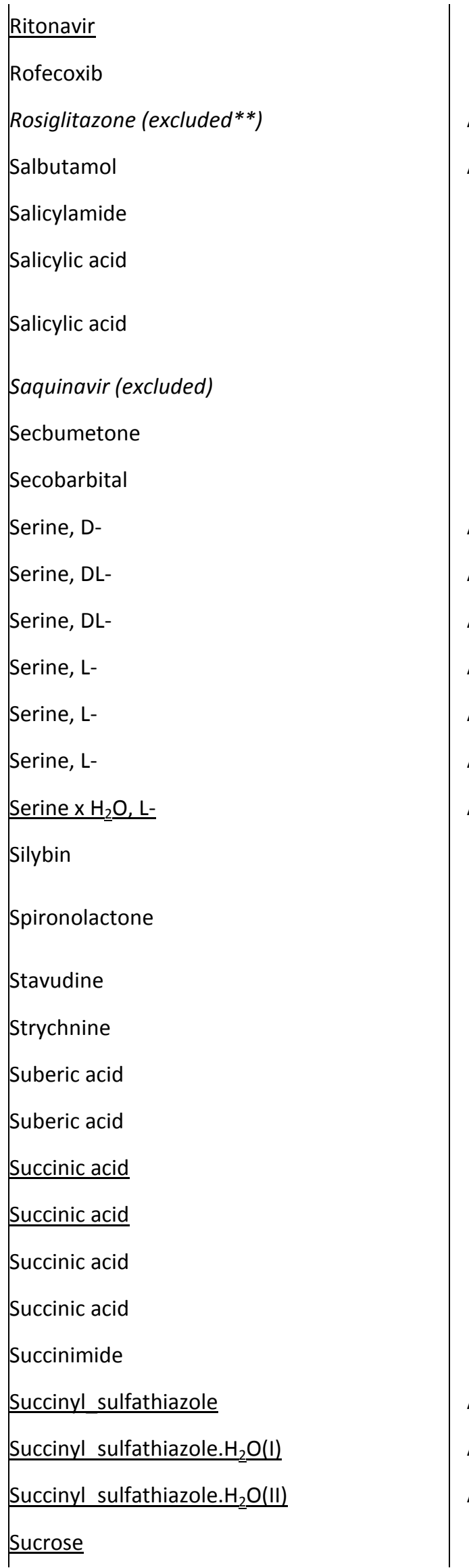

\begin{tabular}{|c|c|c|c|c|c|c|c|c|c|}
\hline B & 21 & 10 & 168 & 0.88 & 3.14 & 5.05 & 3.69 & 5.55 & [188] \\
\hline $\mathrm{N}$ & -15 & 18 & 207 & 0.00 & 1.15 & 2.43 & 1.66 & 2.23 & {$[8,176,399]$} \\
\hline$A B$ & 80 & 17 & 123 & 0.34 & 1.88 & 2.64 & 2.55 & 2.61 & {$[400,401]$} \\
\hline$A B$ & 0 & 4 & 151 & 1.19 & 1.82 & 1.26 & 1.43 & 1.98 & [402] \\
\hline A & 37 & 29 & 140 & 0.62 & 0.61 & 1.57 & 1.16 & 1.03 & [403] \\
\hline A & 24 & 28 & 158 & 0.70 & 0.40 & 1.10 & 0.91 & 0.99 & [43] \\
\hline A & 25 & 28 & 158 & 0.70 & 0.40 & 1.10 & 0.91 & 0.99 & $\begin{array}{c}{[32,34,44,68,71,} \\
82,140,404]\end{array}$ \\
\hline B & -22 & 19 & 350 & 1.46 & 3.89 & 5.55 & 4.09 & 5.30 & {$[163,310]$} \\
\hline B & 0 & 9 & 86 & 0.26 & 1.16 & 1.28 & 1.19 & 1.84 & [405] \\
\hline A & 30 & 24 & 98 & 0.52 & 1.30 & 1.41 & 1.11 & 1.90 & [103] \\
\hline$A B$ & 15 & 13 & 220 & 1.03 & 1.30 & 1.15 & 0.60 & 0.76 & [121] \\
\hline$A B$ & 21 & 14 & 240 & 1.03 & 1.30 & 1.15 & 0.60 & 0.76 & [51] \\
\hline$A B$ & 23 & 14 & 240 & 1.03 & 1.30 & 1.15 & 0.60 & 0.76 & [121] \\
\hline$A B$ & 12 & 13 & 222 & 1.03 & 1.30 & 1.15 & 0.60 & 0.76 & [51] \\
\hline$A B$ & 16 & 14 & 246 & 1.03 & 1.30 & 1.15 & 0.60 & 0.76 & {$[96,97]$} \\
\hline$A B$ & 38 & 13 & 220 & 1.03 & 1.30 & 1.15 & 0.60 & 0.76 & [98] \\
\hline$A B$ & 16 & 12 & 222 & 1.34 & 1.58 & 1.38 & 0.70 & 0.93 & [51] \\
\hline A & 33 & 28 & 174 & 1.39 & 2.58 & 3.57 & 3.57 & 3.25 & [406] \\
\hline $\mathrm{N}$ & 3 & 18 & 135 & 0.00 & 1.63 & 3.81 & 2.24 & 3.17 & $\begin{array}{c}{[32,68,148,161,} \\
300]\end{array}$ \\
\hline A & 19 & 27 & 160 & 0.47 & 1.65 & 1.96 & 1.55 & 1.56 & [407] \\
\hline B & 29 & 25 & 275 & 0.00 & 1.68 & 1.99 & 2.62 & 2.41 & [408] \\
\hline A & 27 & 26 & 142 & 1.14 & 0.70 & 1.08 & 0.33 & 1.39 & [126] \\
\hline A & 35 & 26 & 142 & 1.14 & 0.70 & 1.08 & 0.33 & 1.39 & [43] \\
\hline A & 29 & 27 & 187 & 0.97 & 0.69 & 1.06 & 0.34 & 0.82 & [91] \\
\hline A & 29 & 27 & 187 & 0.97 & 0.69 & 1.06 & 0.34 & 0.82 & [29] \\
\hline A & 29 & 27 & 187 & 0.97 & 0.69 & 1.06 & 0.34 & 0.82 & [92] \\
\hline A & 29 & 27 & 187 & 0.97 & 0.69 & 1.06 & 0.34 & 0.82 & [43] \\
\hline A & 30 & 24 & 126 & 0.34 & 0.89 & 0.99 & 0.76 & 0.70 & [63] \\
\hline$A B$ & 14 & 25 & 194 & 1.34 & 1.78 & 3.39 & 2.25 & 2.34 & [294] \\
\hline$A B$ & 50 & 25 & 194 & 1.34 & 1.78 & 3.39 & 2.25 & 2.34 & [294] \\
\hline$A B$ & 46 & 25 & 194 & 1.34 & 1.78 & 3.39 & 2.25 & 2.34 & [294] \\
\hline $\mathrm{N}$ & 6 & 3 & 188 & 2.01 & 3.43 & 2.63 & 2.32 & 2.23 & [197] \\
\hline
\end{tabular}




\begin{tabular}{|c|c|c|c|c|c|c|c|c|c|c|}
\hline Sucrose & $\mathrm{N}$ & 1 & 2 & 191 & 2.01 & 3.43 & 2.63 & 2.32 & 2.23 & [409] \\
\hline Sulfadiazine & $A B$ & 38 & 29 & 253 & 0.59 & 1.40 & 2.58 & 2.08 & 1.72 & {$[61,68,410-416]$} \\
\hline Sulfadimethoxine (excluded) & $A B$ & -56 & 22 & 204 & 0.59 & 1.78 & 2.77 & 2.17 & 2.12 & {$[8,171]$} \\
\hline Sulfamerazine & $A B$ & 41 & 27 & 237 & 0.59 & 1.41 & 2.52 & 2.10 & 1.86 & $\begin{array}{c}{[68,139,246,411} \\
415,417]\end{array}$ \\
\hline Sulfamethazine & $A B$ & 48 & 23 & 176 & 0.59 & 1.41 & 2.46 & 2.13 & 2.00 & {$[68,70,415,418]$} \\
\hline Sulfamethizole & $A B$ & 27 & 30 & 208 & 0.59 & 1.26 & 2.71 & 2.17 & 1.79 & {$[8,246]$} \\
\hline Sulfamethoxazole & $A B$ & 27 & 26 & 167 & 0.59 & 1.21 & 2.43 & 1.99 & 1.72 & {$[8,68,73,140]$} \\
\hline Sulfanilacetamide & $A B$ & 12 & 24 & 183 & 0.71 & 1.31 & 2.63 & 1.51 & 1.49 & [8] \\
\hline Sulfanilamide & $A B$ & 44 & 22 & 165 & 0.67 & 1.18 & 1.96 & 1.46 & 1.20 & [419] \\
\hline Sulfanilamide $\mathrm{x}_{2} \mathrm{O}$ & $A B$ & 45 & 23 & 185 & 0.67 & 1.18 & 1.96 & 1.46 & 1.20 & [420] \\
\hline Sulfapyridine (excluded) & $A B$ & 38 & 27 & 192 & 0.59 & 1.32 & 2.54 & 2.04 & 1.76 & [421] \\
\hline Sulfasalazine & $A B$ & 10 & 26 & 220 & 1.06 & 2.21 & 3.42 & 3.00 & 2.70 & {$[139,162,186,205]$} \\
\hline Sulfathiazole & $A B$ & 18 & 29 & 202 & 0.59 & 1.21 & 2.60 & 2.06 & 1.69 & {$\left[\begin{array}{c}{[8,68,73,139,186} \\
411,422,423]\end{array}\right.$} \\
\hline Sulfathiazole (Form $\alpha$ ) & $A B$ & 42 & 29 & 202 & 0.59 & 1.21 & 2.60 & 2.06 & 1.69 & [424] \\
\hline Sulfathiazole (Form $\beta$ ) & $A B$ & 36 & 29 & 202 & 0.59 & 1.21 & 2.60 & 2.06 & 1.69 & [424] \\
\hline Sulfisomidine & $A B$ & 13 & 26 & 243 & 0.59 & 1.44 & 2.49 & 2.12 & 2.00 & {$[32,415,418]$} \\
\hline Sulindac & A & 39 & 32 & 184 & 0.57 & 1.39 & 2.72 & 2.26 & 2.57 & [425] \\
\hline Tartaric acid & A & 16 & 24 & 175 & 1.23 & 1.30 & 1.13 & 0.61 & 0.94 & [91] \\
\hline Tartaric acid & A & 7 & 24 & 175 & 1.23 & 1.30 & 1.13 & 0.61 & 0.94 & [43] \\
\hline Tartaric acid & A & 10 & 24 & 175 & 1.23 & 1.30 & 1.13 & 0.61 & 0.94 & [92] \\
\hline Taurine & $A B$ & 25 & 18 & 300 & 0.52 & 1.34 & 1.64 & 0.49 & 0.83 & [51] \\
\hline Taurine & $A B$ & 22 & 17 & 328 & 0.52 & 1.34 & 1.64 & 0.49 & 0.83 & [63] \\
\hline t-butanol & $\mathrm{N}$ & 4 & 4 & 26 & 0.31 & 0.35 & 0.39 & 0.19 & 0.73 & [426] \\
\hline Temafloxacin & $A B$ & 58 & 18 & $\underline{175}$ & 0.73 & 1.81 & 2.87 & 2.39 & 2.77 & [104] \\
\hline Terephthalic acid & A & -7 & 32 & 300 & 1.14 & 0.77 & 1.46 & 0.94 & 1.15 & [43] \\
\hline Terephthalic acid & A & 1 & 32 & 300 & 1.14 & 0.77 & 1.46 & 0.94 & 1.15 & [131] \\
\hline Testosterone & $\mathrm{N}$ & 8 & 14 & 154 & 0.31 & 1.01 & 2.27 & 1.55 & 2.38 & \begin{tabular}{|c}
{$[32,79,99,147$} \\
$148,159,161,205$ \\
$305]$
\end{tabular} \\
\hline Testosterone & $\mathrm{N}$ & 23 & 14 & 155 & 0.31 & 1.01 & 2.27 & 1.55 & 2.38 & [219] \\
\hline Testosterone Propionate (excluded ${ }^{* *}$ ) & $\mathrm{N}$ & 15 & 8 & 120 & 0.00 & 1.07 & 2.40 & 1.41 & 2.82 & {$[32,99]$} \\
\hline Thalidomide & A & 18 & 32 & 275 & 0.34 & 1.72 & 2.53 & 2.01 & 1.75 & [427] \\
\hline
\end{tabular}




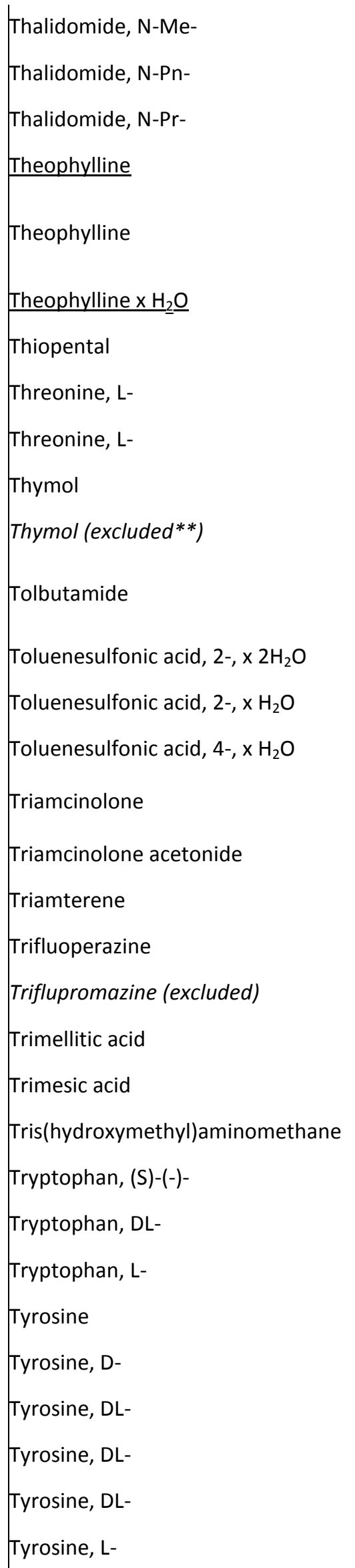

\begin{tabular}{|c|c|c|c|c|c|c|c|c|c|}
\hline $\mathrm{N}$ & 32 & 18 & 159 & 0.00 & 1.77 & 2.55 & 2.03 & 1.89 & [427] \\
\hline $\mathrm{N}$ & 35 & 11 & 105 & 0.00 & 1.79 & 2.56 & 2.02 & 2.45 & [427] \\
\hline $\mathrm{N}$ & 4 & 15 & 136 & 0.00 & 1.78 & 2.55 & 2.03 & 2.17 & [427] \\
\hline A & 31 & 31 & 272 & 0.35 & 1.29 & 1.99 & 1.46 & 1.22 & [294] \\
\hline A & 13 & 31 & 273 & 0.35 & 1.29 & 1.99 & 1.46 & 1.22 & $\begin{array}{c}{[8,33,68,70,86,} \\
224,305,404,428]\end{array}$ \\
\hline$A$ & 45 & 30 & 272 & 0.66 & 1.58 & 2.21 & 1.56 & 1.39 & [294] \\
\hline$A$ & 36 & 28 & 158 & 0.51 & 1.34 & 2.00 & 1.49 & 1.90 & [429] \\
\hline$A B$ & 10 & 14 & 270 & 1.03 & 1.33 & 1.14 & 0.61 & 0.91 & [98] \\
\hline$A B$ & 12 & 13 & 256 & 1.03 & 1.33 & 1.14 & 0.61 & 0.91 & {$[32,97]$} \\
\hline$A$ & 16 & 24 & 50 & 0.50 & 0.42 & 0.78 & 0.84 & 1.34 & [207] \\
\hline A & 10 & 24 & 52 & 0.50 & 0.42 & 0.78 & 0.84 & 1.34 & {$[32,139]$} \\
\hline A & 17 & 28 & 129 & 0.59 & 1.15 & 2.21 & 1.33 & 2.06 & $\begin{array}{c}{[8,246,401,430,} \\
431]\end{array}$ \\
\hline A & 5 & 28 & $\underline{199}$ & 0.31 & 0.88 & 1.72 & 0.89 & 1.20 & [432] \\
\hline A & -1 & 27 & $\underline{179}$ & 0.31 & 0.88 & 1.72 & 0.89 & 1.20 & [432] \\
\hline A & 5 & 26 & 125 & 0.31 & 0.88 & 1.72 & 0.89 & 1.20 & [432] \\
\hline $\mathrm{N}$ & 20 & 16 & 270 & 1.03 & 2.25 & 3.21 & 2.27 & 2.83 & {$[68,147,148,213]$} \\
\hline $\mathrm{N}$ & 15 & 14 & 293 & 0.56 & 2.14 & 3.13 & 2.18 & 3.15 & [433] \\
\hline B & 16 & 44 & 313 & 0.68 & 1.45 & 2.64 & 3.19 & 1.83 & {$[68,434]$} \\
\hline B & 22 & 8 & 25 & 0.00 & 1.42 & 1.79 & 2.17 & 2.89 & {$[25,182,435]$} \\
\hline B & 120 & 10 & 25 & 0.00 & 0.94 & 1.51 & 1.79 & 2.48 & {$[25,182,187]$} \\
\hline A & 34 & 30 & 219 & 1.71 & 1.10 & 1.84 & 1.14 & 1.36 & [131] \\
\hline A & 46 & 34 & 375 & 1.71 & 1.10 & 1.84 & 1.14 & 1.36 & [131] \\
\hline B & 17 & 11 & 172 & 1.01 & 1.62 & 1.16 & 0.82 & 0.95 & [436] \\
\hline$A B$ & 12 & 24 & 283 & 1.09 & 1.23 & 1.80 & 1.62 & 1.54 & [98] \\
\hline$A B$ & 18 & 24 & 289 & 1.09 & 1.23 & 1.80 & 1.62 & 1.54 & [437] \\
\hline$A B$ & 11 & 24 & 283 & 1.09 & 1.23 & 1.80 & 1.62 & 1.54 & {$[51]$} \\
\hline$A B$ & 19 & 22 & 343 & 1.28 & 1.29 & 1.60 & 1.18 & 1.37 & {$[32,37,438-440]$} \\
\hline$A B$ & 27 & 20 & 312 & 1.28 & 1.29 & 1.60 & 1.18 & 1.37 & [387] \\
\hline$A B$ & 25 & 26 & 325 & 0.50 & 0.70 & 1.42 & 1.24 & 1.37 & [51] \\
\hline$A B$ & 26 & 26 & 325 & 0.50 & 0.70 & 1.42 & 1.24 & 1.37 & [37] \\
\hline$A B$ & 28 & 21 & 325 & 0.50 & 0.70 & 1.42 & 1.24 & 1.37 & [387] \\
\hline$A B$ & 23 & 22 & 319 & 1.28 & 1.29 & 1.60 & 1.18 & 1.37 & [80] \\
\hline
\end{tabular}




\begin{tabular}{|l|c|c|c|c|c|c|c|c|c|c|} 
Tyrosine, L- & $\mathrm{AB}$ & $\mathbf{2 5}$ & 20 & 319 & 1.28 & 1.29 & 1.60 & 1.18 & 1.37 & {$[51]$} \\
\hline Urea & $\mathrm{N}$ & $\mathbf{1 2}$ & 17 & 133 & 0.72 & 0.69 & 1.17 & 0.63 & 0.47 & {$[115]$} \\
\hline Urea & $\mathrm{N}$ & $\mathbf{1 5}$ & 17 & 133 & 0.72 & 0.69 & 1.17 & 0.63 & 0.47 & {$[197]$} \\
\hline Uric acid & $\mathrm{A}$ & $\mathbf{2 7}$ & 31 & 300 & 0.80 & 1.58 & 2.36 & 1.48 & 1.00 & $\underline{[285]}$ \\
Uric acid $\mathrm{x} 2 \mathrm{H}_{2} \mathrm{O}$ & $\mathrm{A}$ & $\mathbf{4 2}$ & 32 & 320 & 0.80 & 1.58 & 2.36 & 1.48 & 1.00 & {$[109]$} \\
Ursocholic acid & $\mathrm{A}$ & $\mathbf{4}$ & 27 & $\underline{157}$ & 1.51 & 1.66 & 2.66 & 1.82 & 3.31 & {$[190]$} \\
Valine, D- & $\mathrm{AB}$ & $\mathbf{2}$ & 14 & 295 & 0.78 & 0.97 & 0.92 & 0.39 & 0.99 & {$[51]$} \\
Valine, DL- & $\mathrm{AB}$ & $\mathbf{7}$ & 14 & 296 & 0.78 & 0.97 & 0.92 & 0.39 & 0.99 & {$[51]$} \\
\hline Valine, L- & $\mathrm{AB}$ & $\mathbf{4}$ & 14 & 311 & 0.78 & 0.97 & 0.92 & 0.39 & 0.99 & {$[51]$} \\
Vanillin & $\mathrm{A}$ & $\mathbf{2 2}$ & 25 & $\mathbf{8 2}$ & 0.44 & 0.76 & 1.46 & 1.02 & 1.13 & {$[197]$} \\
\hline Vidarabine & $\mathrm{B}$ & $\mathbf{4 3}$ & 29 & 234 & 0.97 & 2.22 & 2.64 & 2.69 & 1.75 & {$[69]$} \\
Xanthine & $\mathrm{A}$ & $\mathbf{4 7}$ & 32 & 300 & 0.89 & 1.14 & 1.75 & 1.47 & 0.94 & {$[8,68]$} \\
\hline
\end{tabular}

${ }^{a}$ Compound names: underlined = calorimetric data; italic = excluded data; double asterisk = "n=2, different labs" data (see text).

${ }^{\mathrm{b}} \mathrm{A}=$ acid, $\mathrm{B}=$ base, $\mathrm{AB}=$ ampholyte/zwitterion, $\mathrm{N}=$ nonionizable molecule.

${ }^{C}$ Underlined $\mathrm{mp}$ are predicted using Lang-Bradley program [19, 20].

d Underlined references were cited in Yalkowsky et al. [8]. the Creative Commons Attribution license (http://creativecommons.org/licenses/by/3.0/) (cc) BY 